\title{
WestVirginiaUniversity
}

THE RESEARCH REPOSITORY @ WVU

Graduate Theses, Dissertations, and Problem Reports

2015

\section{Role of Mitochondria in TNF-alpha Neurotoxicity in Stroke}

Danielle N. Doll

Follow this and additional works at: https://researchrepository.wvu.edu/etd

\section{Recommended Citation}

Doll, Danielle N., "Role of Mitochondria in TNF-alpha Neurotoxicity in Stroke" (2015). Graduate Theses, Dissertations, and Problem Reports. 5502.

https://researchrepository.wvu.edu/etd/5502

This Dissertation is protected by copyright and/or related rights. It has been brought to you by the The Research Repository @ WVU with permission from the rights-holder(s). You are free to use this Dissertation in any way that is permitted by the copyright and related rights legislation that applies to your use. For other uses you must obtain permission from the rights-holder(s) directly, unless additional rights are indicated by a Creative Commons license in the record and/ or on the work itself. This Dissertation has been accepted for inclusion in WVU Graduate Theses, Dissertations, and Problem Reports collection by an authorized administrator of The Research Repository @ WVU.

For more information, please contact researchrepository@mail.wvu.edu. 


\title{
Role of Mitochondria in TNF-alpha Neurotoxicity in Stroke
}

\author{
Danielle N. Doll
}

A dissertation submitted to the West Virginia University Health Science Center Neuroscience Program in partial fulfillment of the requirements for the degree of:

Doctor of Philosophy in Neuroscience

\author{
Committee: \\ James W. Simpkins, Ph.D., Chair \\ Rosana Schafer, Ph.D. \\ Taura L. Barr, Ph.D. \\ Paul D. Chantler, Ph.D. \\ Xuefang Ren, Dr. Med. \\ Valeriya Gritsenko, Ph.D. \\ WVU Neuroscience Program \\ Morgantown, West Virginia \\ 2015
}

Keywords: Stroke, TNF-alpha, Mitochondria, LPS, Infection

Copyright 2015 Danielle N. Dol 


\section{ABSTRACT \\ Role of Mitochondria in TNF-alpha Neurotoxicity in Stroke}

\section{Danielle N. Doll}

TNF-alpha is known to exacerbate infarct volume in animal models and elevated levels of TNFalpha are correlated with worse outcomes in stroke patients. However, the mechanism is not well understood. TNF-alpha has been shown to be neurotoxic at high doses and after long exposure times which is not clinically relevant. Thus, we show for the first time that TNF-alpha at clinically relevant concentrations seen in the serum of stroke patients rapidly and profoundly decreases mitochondrial function resulting in neuronal cell death through activation of caspase 8 and cytochrome $c$ release. A slight decrease in mitochondrial function is detrimental to neurons due to their high energy demand. Since TNF-alpha is known to be increased during ischemia and correlated with negative outcomes, it is important to understand the neurotoxic mechanism of TNF-alpha to develop potential therapeutic targets for stroke. Moreover, TNF-alpha is increased during infections, and 30-40\% of strokes occur during an active infection. We show that having a stroke during an active bacterial infection mimic results in an increased infarct volume, worsened neurological deficits, and prolonged sickness behavior. TNF-alpha's rapid and profound effect on mitochondrial function may be one mechanism by which stroke severity is exacerbated, neurological deficits are worsened, and recovery delayed. Thus, enhancing mitochondrial function acutely post-stroke could be a potential therapeutic intervention. 


\section{Dedication}

This dissertation is in memory of my late father, Terry Doll. Who I am today, what I have accomplished, and how successful I will be in the future are all due to having a remarkable, loving, and kind-hearted dad like you. Without you, this accomplishment would not have been possible.

This dissertation is dedicated to my mother, Madlene Doll. You have been my personal cheer leader and my biggest critic all my life. Your immense support and never ending love paved the path to this accomplishment. Thank you for always being there.

This dissertation is also dedicated to my fiancé, Tim Sims. When life gets crazy and appears to be a little too much to handle, you are a constant that always keeps me grounded. Your love and goofiness provides me with endless smiles and laughs. Thank you for loving and supporting me through everything. 


\section{Acknowledgments}

I would like to thank my mentor and advisor Dr. James W. Simpkins. His constant support and belief in me aided in my success as a graduate student and provided me with the tools to be a successful independent scientist.

I would also like to thank my committee members, Dr. Rosana Schafer, Dr. Taura Barr, Dr. Paul Chanter, Dr. Xuefang Ren, and Dr. Valeriya Gritsenko for their support and insight.

Lastly, I would like to thank current and past members of the Simpkins lab with a special thanks to Sara Lewis, Stephanie Rellick, Liz Engler-Chiurazzi, Sophie Ren, and Heng Hu. With your help, I was able to succeed. 


\section{Table of Contents}

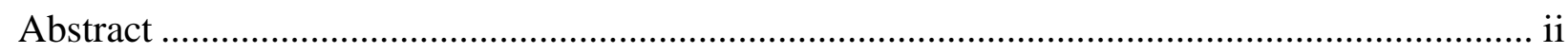

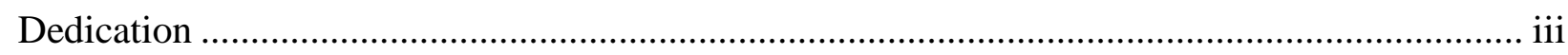

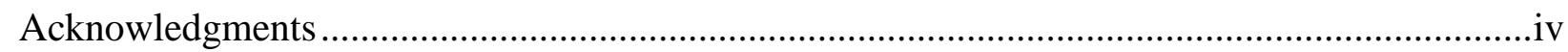

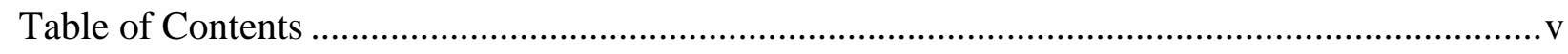

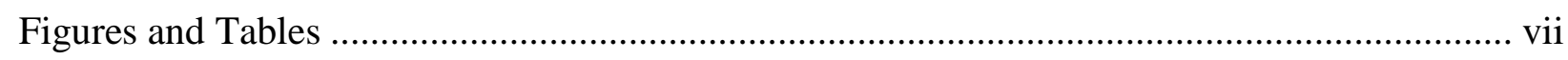

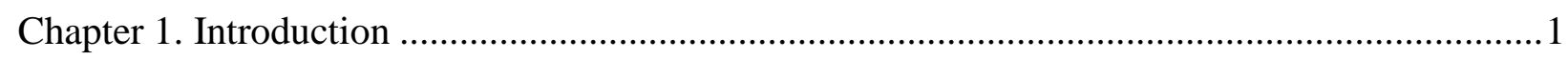

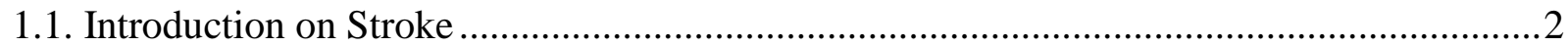

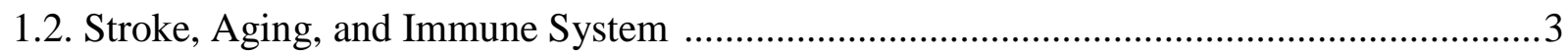

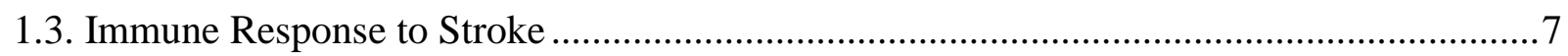

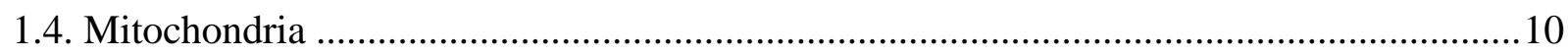

1.5. Cytokine Changes Following Stroke ......................................................................... 12

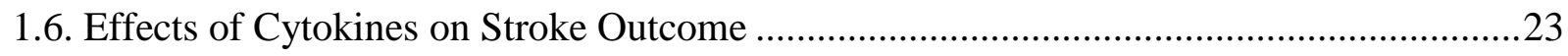

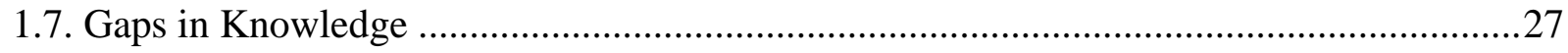

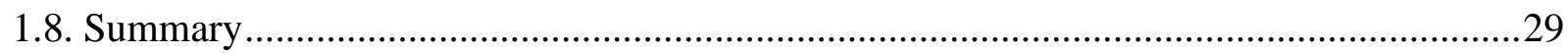

Chapter 2. Rapid Mitochondrial Dysfunction Mediates TNF-alpha Induced Neurotoxicity........32

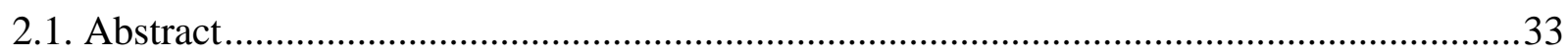

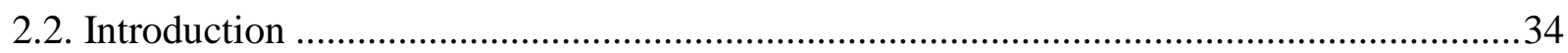

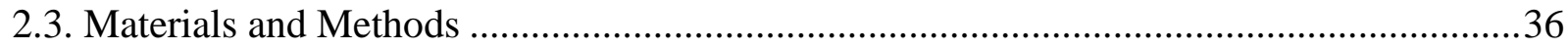

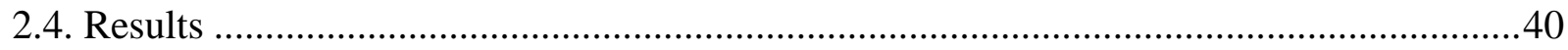

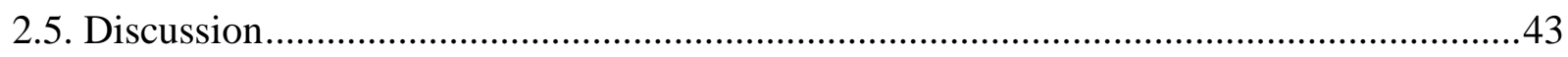

Chapter 3. Mitochondrial Crisis in Cerebrovascular Endothelial Cells Opens the Blood-Brain

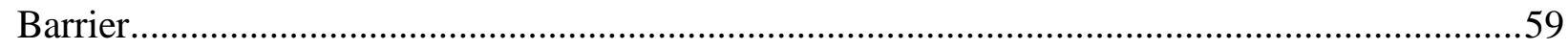

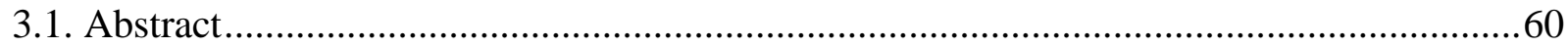

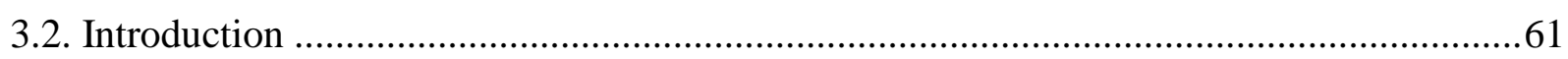

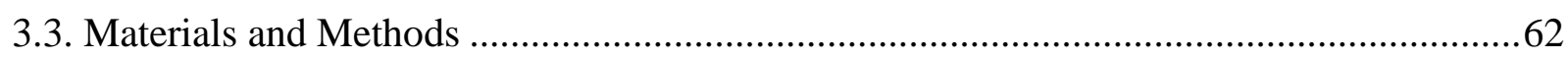

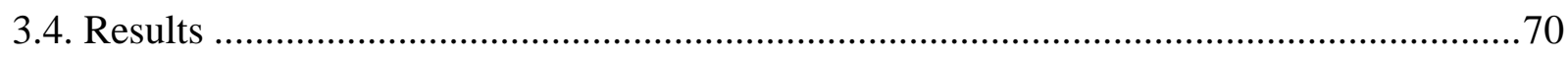

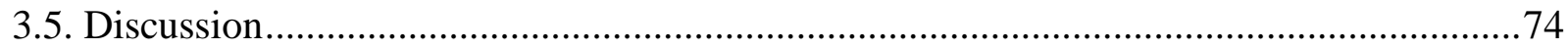


Chapter 4. Lipopolysaccharide Exacerbates Infarct Size and Results in Worsened Post-Stroke

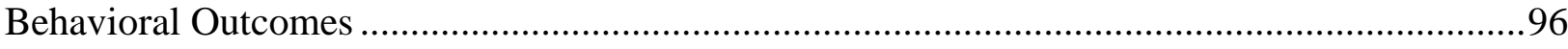

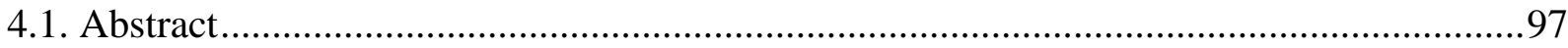

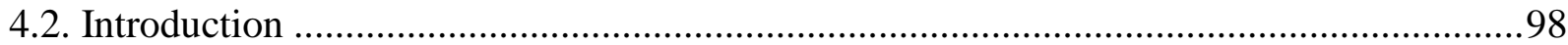

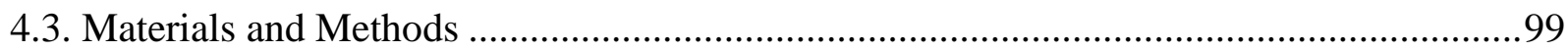

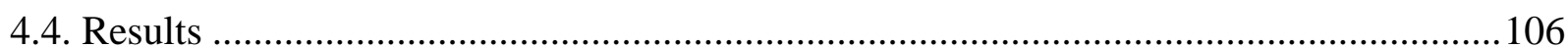

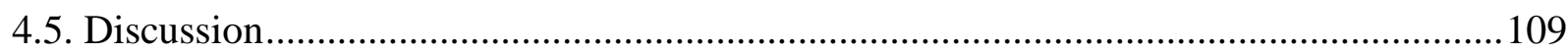

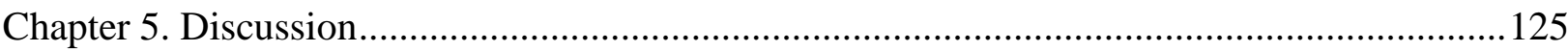

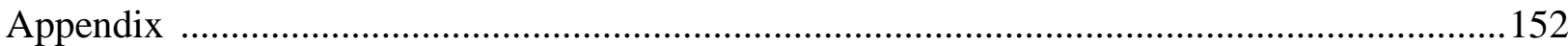

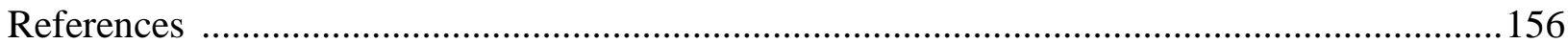




\section{Figures and Tables}

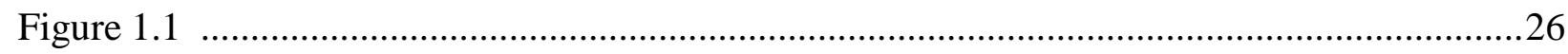

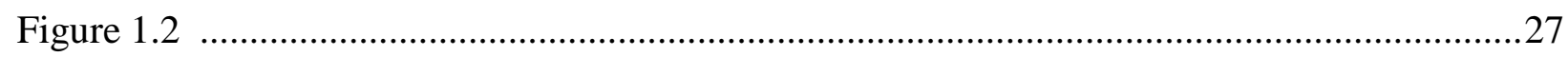

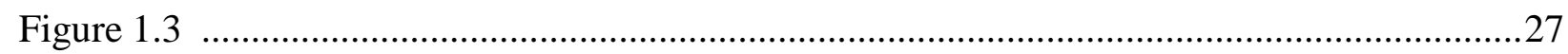

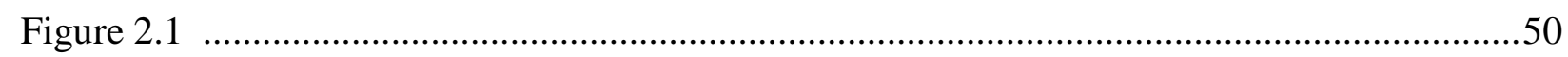

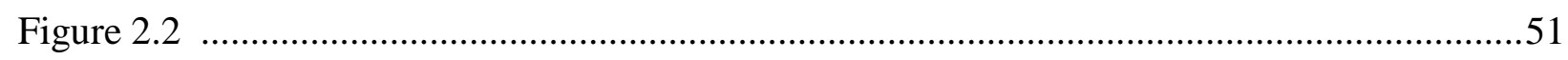

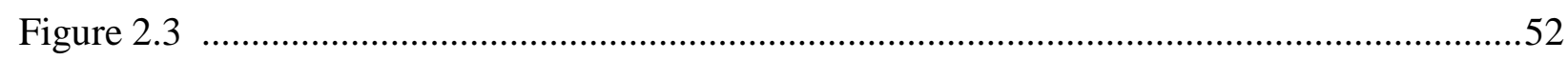

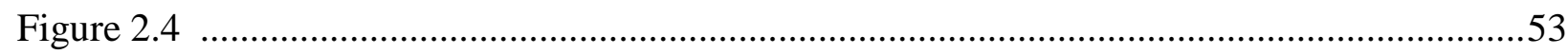

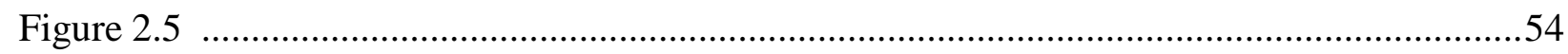

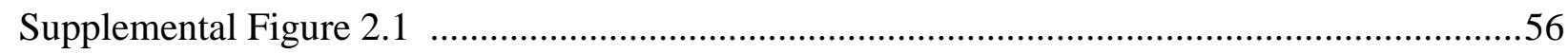

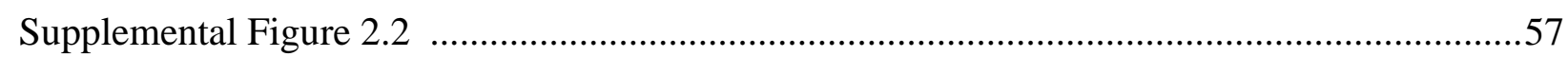

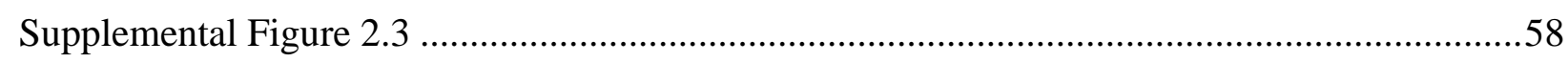

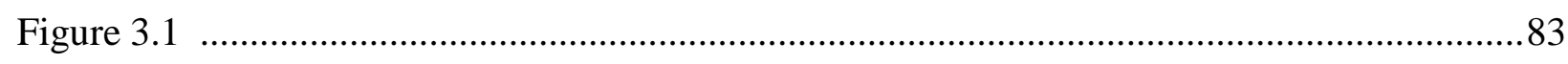

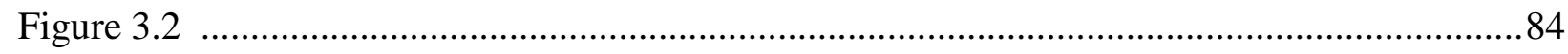

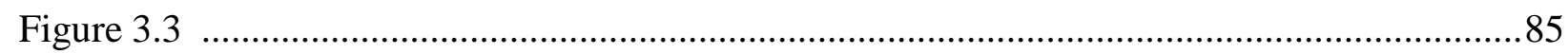

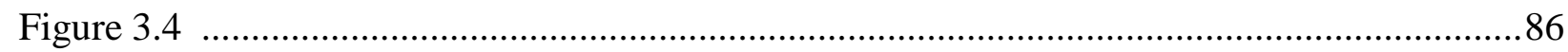

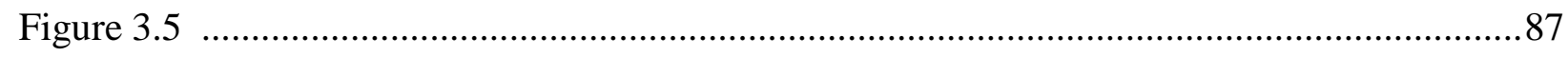

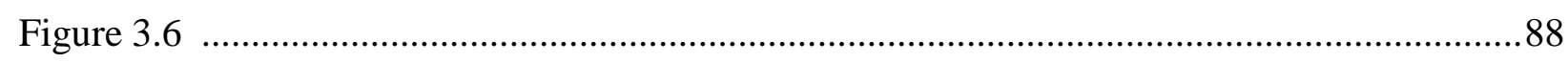

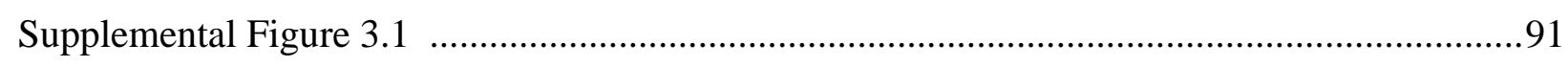

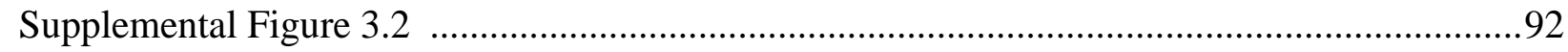

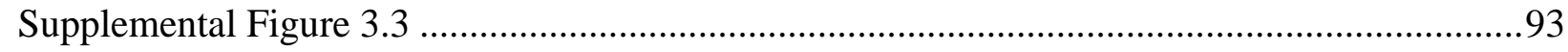

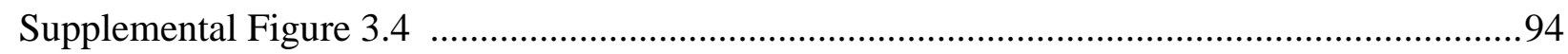

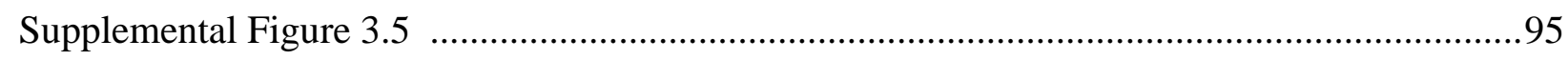

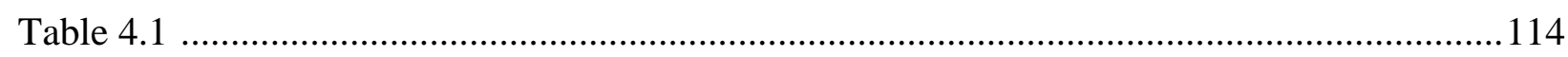

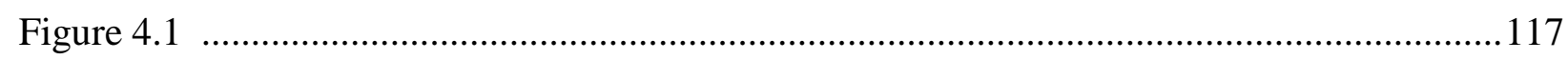

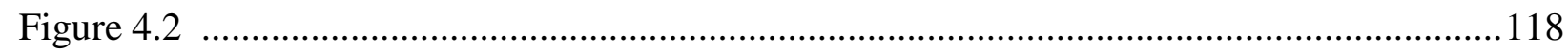

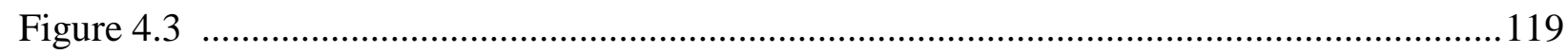

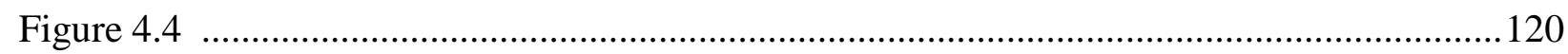

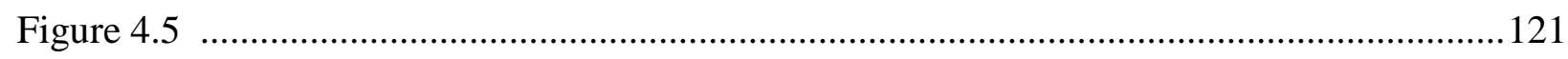

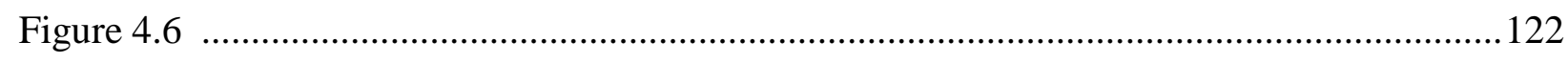

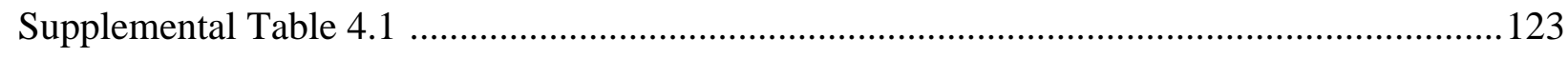

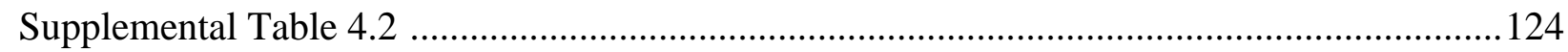


Chapter 1

\section{Introduction}




\subsection{Introduction on Stroke}

Approximately 800,000 Americans suffer from a stroke each year, and the American Heart Association predicts a 5.1 percent increase in stroke cases for Americans between 45 and 64 years of age by 2030. More than 137,000 people die from a stroke each year resulting in stroke being the fifth leading cause of death and leading cause of disability in the United States (American Heart Association, 2012). Ischemic strokes which result from an interruption of blood flow to the brain due to a clot, account for 87 percent of all stroke cases (American Heart Association, 2012). Although ischemic stroke is a devastating problem in the United States and worldwide, the only FDA approved treatment is tissue plasminogen activator (tPA), which is a thrombolytic to break up the clot. Unfortunately, less than 5 percent of patients receive tPA due to its limited time window of 4.5 hours; thus, most patients only receive supportive care (Fonarow et al., 2011).

An occlusion of a cerebral artery causes deprivation of oxygen, glucose, and lipids resulting in necrotic brain tissue. Breakdown of the blood-brain barrier and activation of inflammatory cells in the brain and in the periphery result in inflammation in the necrotic tissue (Davies et al., 1999; Zheng and Yenari, 2004). Inflammatory mechanisms both in the periphery and in the central nervous system (CNS) are important in promoting brain inflammation by producing inflammatory mediators such as cytokines and clearing away dead tissue after cerebral ischemia (Danton and Dietrich, 2003). Cytokines are key players in the inflammatory mechanism and contribute to the progression of ischemic damage (Liu et al., 1994). The discovery of biomarkers and novel therapeutics for stroke has been the focus of extensive research recently; thus, understanding the roles of pro-inflammatory and anti-inflammatory cytokines that are produced during stroke will help us further understand how inflammation contributes to the 
progression of ischemic damage and provide potential targets for novel therapeutics and biomarkers for prognosis of stroke.

\subsection{Stroke, Aging, and Immune System}

It was once thought that the brain was an immune-privileged organ and did not produce an inflammatory response to ischemic brain injury (Arvin et al., 1996). However, it is now well established that cells in the brain produce and secrete cytokines, chemokines and adhesion molecules enabling the brain to mount a central and peripheral inflammatory response to ischemia (Liu et al., 1994; Ferrarese et al., 1999). Both central and peripheral inflammation are key contributors to the pathophysiology of stroke (Dirnagl et al., 1999; Wang et al., 2007a). There is increasing evidence that peripheral inflammation not only plays a role in the pathology of stroke but impacts stroke etiology by increasing susceptibility (Emsley and Hopkins, 2008). Risk factors such as atherosclerosis, obesity, diabetes, and hypertension are all associated with an increased peripheral inflammatory profile, and the majority of stroke patients have at least one of these risk factors (Hankey, 2006). Many of these factors could participate in regulating interactions between the central and peripheral immune systems; those classically identified as immune response mediators and those traditionally unassociated. A further understanding of the interaction between the immune and nervous system is important to determine the contribution of mal-adaptive immune responses as a causative factor resulting in poor functional outcome.

Stroke most commonly occurs when an atherosclerotic plaque ruptures resulting in thromboembolism. The initiation and progression of plaques are driven by inflammatory cells and mediators such as cytokines and chemokines (Hansson and Libby, 2006). Dysfunctional endothelial cells of the vascular wall at the site of the atherosclerotic lesion express adhesion 
molecules such as VCAM-1 and ICAM-1 that recruit macrophages and T cells (Cybulsky et al., 2001). These immune cells produce cytokines and chemokines along with vasoactive molecules that activates immune cells and smooth muscle cell proliferation resulting in the progression of the atherosclerotic lesion (McColl et al., 2009). The continuous recruitment of immune cells and activation results in the formation of a mature plaque, and activated macrophages and $\mathrm{T}$ cells are significant part of the plaque (Zhou et al., 2000). These activated immune cells secrete matrix metalloproteinases (MMPs) which are collagen-degrading proteases that destabilize the plaque and results in the rupture of the plaque causing ischemia (Johnson et al., 2005). It is clear that inflammation is an essential contributor to the development and progression of atherosclerosis, and numerous pre-clinical studies show that animal models deficient in adhesion molecules, inflammatory mediators, and $\mathrm{T}$ cells attenuate the progression of plaque formation and the instability of the plaque (Boring et al., 1998; Dong et al., 2000; Zhou et al., 2000; Chi et al., 2004). Clinical studies using positron emission tomography (PET) have shown patients presenting with transient ischemic attack have high ${ }^{18} \mathrm{~F}$-fluoreodeoxyglucose (FDG) signal in atherosclerotic plaques (Rudd et al., 2002; Davies et al., 2005). FDG is taken up by macrophages and indicates the degree of macrophage activation, which further supports inflammation causing plaque instability (Warburton and Gillard, 2006).

Increased atherosclerotic burden is a hallmark of obesity and diabetes, and these co-morbid diseases cause endothelial dysfunction through insulin resistance and inflammation (Hunt and Jurd, 1997). Thus, risk factors such as obesity and diabetes increase stroke susceptibility through pro-atherogenic effects. Furthermore, clinical studies have linked peripheral bacterial infections and increased stroke susceptibility during the first 3 days after infection (Grau et al., 1998; Smeeth et al., 2004). Infection is known to increase vascular disease, along with 
increasing inflammatory mediators such as cytokines to clear the infection, which can promote the formation of plaques and increase destabilization of the plaque (Li et al., 2002; Michelsen et al., 2004). Along with pro-atherogenic effects of infection, infection promotes a hypercoagulable state (Vila et al., 2000b). Interleukin (IL)-6 and tumor necrosis factor- $\alpha$ (TNF$\alpha)$ are cytokines increased during the inflammatory response to infection that activate the tissue factor-mediated extrinsic pathway of coagulation, and clinical studies have observed in stroke patients with infections, increased platelet activation and decreased concentrations of anticoagulatory molecules such anti-thrombin, activated protein $\mathrm{C}$, and free protein $\mathrm{S}$ (van der Poll et al., 1990; van der Poll et al., 1994) Pre-clinical studies confirm these findings and demonstrate that systemic administration of pro-inflammatory cytokines mimic characteristics of infection. Obese animal models and animal models with other co-morbid diseases such as diabetic mice and spontaneously hypertensive rats exhibit larger infarcts and more severe neurological deficits (McGill et al., 2005; Terao et al., 2008). These pre-clinical and clinical studies support the significance of peripheral inflammation and its impact on stroke and demonstrate how the interaction between the immune system and the brain can affect neurological dysfunction after an ischemic insult.

Not only does acute and chronic inflammation preceding stroke increase susceptibility to stroke and increase brain damage after stroke, but stroke itself initiates a local inflammatory response in the brain and peripheral inflammation that can amplify this central inflammatory reaction. Interruption of cerebral blood flow results in the deprivation of oxygen, glucose, and other essential nutrients to the brain and leads to a complex, multifaceted cascade of inflammatory events. 
It is evident that stroke is a vascular disease that results in neurological deficits after an ischemic insult, and it is not surprising that the prevalence of stroke increases with age because aging affects the vasculature and the immune system. Capillary networks and density decrease during aging in the cortex of adult humans and animals (Brown et al., 2007b; Brown and Thore, 2011). Chronic inflammation and acute infection contribute to capillary disruption and cell loss resulting in a leaky $\mathrm{BBB}$, and aged rodents exhibit increased $\mathrm{BBB}$ disruption along with reduced neurogenesis after stroke (Darsalia et al., 2005; DiNapoli et al., 2008; Ingraham et al., 2008). In addition, age-associated immune system dysfunction increases incidence of acute infections, and co-morbid factors characterized by inflammation such as diabetes and atherosclerosis, and as discussed above, these co-morbid factors greatly increase susceptibility to stroke and worsen outcome after stroke (Castelo-Branco and Soveral, 2014).

Aged individuals are in a chronic state of low grade inflammation referred to as inflamm-aging, and during inflamm-aging there is a shift in cytokines toward a pro-inflammatory state producing pro-inflammatory cytokines such as TNF- $\alpha$ and IL-1 (Bruunsgaard et al., 1999; Griffin, 2006). The immune dysregulation theory supports the idea that during healthy aging anti-inflammatory mediators such as IL-10 inhibits the pro-inflammatory state; however, individuals' systems that are challenged with acute infections, co-morbid factors, genetic predisposition, and environmental factors are unable to mount an anti-inflammatory response to the shift in a pro-inflammatory state resulting in an increase incidence of infections, co-morbid diseases, and stroke (Lio et al., 2004; Castelo-Branco and Soveral, 2014). Thus, understanding the effect of age alone on stroke is difficult because it is likely that age is a surrogate for increased chronic inflammation associated with co-morbid diseases. This creates a pro- 
atherogenic and pro-coagulable state responsible for the increased incidence of stroke in the aged.

\subsection{Immune Response to Stroke}

The complex, multifaceted cascade of events that results from brain deprivation of oxygen, glucose, and other essential nutrients to the brain causes dysfunction of the neurovascular unit (del Zoppo, 2006). Acutely after ischemia, microglia are activated due to the increase in extracellular ATP from the depolarization of neurons and glia, and activated microglia secrete cytokines and chemokines along with developing phagocytic properties (Melani et al., 2005). Within 4-6 hours after an ischemic insult, peripheral leukocytes migrate and adhere to vessel walls and infiltrate into the ischemic brain tissue producing deleterious inflammatory mediators (Wang et al., 2007a). Numerous studies have shown that neutrophils are the first blood-derived cells from the periphery to infiltrate ischemic brain tissue, and when neutrophil infiltration is inhibited, infarct size is significantly decreased (Chopp et al., 1996; Hallenbeck, 1996; Yenari et al., 1998). Reperfusion of the occluded vessel generates reactive oxygen species (ROS) resulting in further activation of immune and brain cells and oxidative stress, and these activated cells secrete cytokines, chemokines, MMPs, nitric oxide (NO), more ROS, and adhesion molecules that increase cell death and disruption of the blood-brain barrier (BBB) (Coyle and Puttfarcken, 1993; Melani et al., 2005). Moreover, reperfusion and disruption of the BBB leads to further leukocyte infiltration into the brain amplifying the inflammatory response and enhancing damage. The peripheral and central inflammatory response after ischemia leads to an increase in neuronal death, infarct size, and severity of stroke. 
Adhesion molecules are essential for leukocytes to infiltrate into the brain after stroke, and notably ICAM-1 and VCAM-1, the same adhesion molecules that recruit immune cells to atherosclerotic lesions, mediate the interaction between leukocytes and the vascular endothelium (DeGraba, 1998). Furthermore, both ICAM-1 and VCAM-1 expression increases after stimulation by cytokines such as TNF- $\alpha$ and IL-1 which are increased after stroke and in obese, diabetic, and atherosclerotic patients (Wang et al., 2007a). An experimental stroke study in diabetic rats showed higher expression of ICAM-1 in diabetic rats after stroke compared to nondiabetic rats, and this increase in adhesion molecules due to co-morbid diseases and infection could contribute to the exacerbation of stroke and increase susceptibility to stroke (Ding et al., 2005). Moreover, using ICAM-1 deficient mice and inhibiting ICAM-1 and VCAM-1in experimental stroke studies result in smaller infarct sizes, and clinically increased plasma and cerebral spinal fluid levels of ICAM-1 and VCAM-1 are observed in stroke patients and correlated with stroke severity (Connolly et al., 1996; Kitagawa et al., 1998; Selakovic et al., 2003; Ehrensperger et al., 2005). Thus, peripheral inflammation due to infection or other comorbid factors may increase stroke severity and stroke susceptibility by increasing adhesion molecules and other inflammatory mediators that produce an environment that enhances leukocyte activation and adhesion.

Furthermore, brain and immune cells produce reactive oxygen species (ROS), and restoration of blood flow in the occluded vessel generates additional ROS (Coyle and Puttfarcken, 1993). ROS activate endothelial cells and cause oxidative stress (Melani et al., 2005; Schock et al., 2007). Oxidative stress and the induction of the inflammatory cascade leads to the breakdown of the blood-brain barrier allowing activated blood-borne immune cells such as neutrophils and T-cells to infiltrate and accumulate in the ischemic brain tissue (Melani et al., 2005). Along with 
the accumulation of activated immune cells from the periphery, microglia in the brain become activated after cerebral ischemia due to the increase in extracellular ATP from the depolarization of neurons and glia and the release through damaged plasma membranes of dying cells (Melani et al., 2005). Activated microglia secrete pro-inflammatory mediators such as cytokines and develop phagocytic and major histocompatibility complex (MHC) class II-restricted antigen presenting characteristics (Davalos et al., 2005). Microglia activation can be beneficial by producing growth factors such as brain-derived neurotrophic factor and clearing away dead tissue and debris after ischemia; however, the release of pro-inflammatory cytokines such as tumor necrosis factor- $\alpha(\mathrm{TNF}-\alpha)$ and production of reactive oxygen species and nitric oxide after microglia activation is detrimental (Cardenas et al., 2002; Tang et al., 2007). ATP is an early danger signal increasing inflammation, but as cells die, molecular signals called danger associated molecular pattern molecules (DAMPs) are generated and activate pattern recognition receptors such as toll like receptors (TLRs) (Marsh et al., 2009). These pattern recognition receptors are found on endothelial cells and microglia in the brain along with infiltrating leukocytes from the periphery, and activation of pattern recognition receptors increases cytokines (Marsh et al., 2009). Moreover as neurons begin to die after ischemia, cell-cell interactions with microglia are lost and further increases inflammatory signaling (Matsumoto et al., 2007). Thus acutely after ischemia, hypoxia and oxidative stress induces synthesis of nuclear factor $\kappa \mathrm{B}(\mathrm{NF}-\kappa \mathrm{B})$, hypoxia inducible factor 1 and many other transcription factors that increase the expression of pro-inflammatory and anti-inflammatory cytokines (Baeuerle and Henkel, 1994). As cells die and brain tissue is damaged, molecular danger signals further potentiate the inflammatory response by activating more microglia and infiltrating leukocytes in a feed-forward response producing more deleterious pro-inflammatory cytokines. This increase in expression of cytokines further increases the expression of adhesion molecules on endothelial 
cells that result in additional recruitment of leukocytes from the periphery (Bargatze et al., 1994). These inflammatory changes after ischemia lead to an increase in neuronal cell death resulting in a larger infract volume and worse outcome. Inflammation is a key player in brain damage during cerebral ischemia; however, inflammation aiding in repair and recovery after cerebral ischemia can be beneficial. Thus, further mechanistic understanding of how inflammation contributes to injury or repair after ischemia is important for discovering potential therapeutic targets for stroke and for using inflammatory mediators such as cytokines as biomarkers for prognosis.

\subsection{Mitochondria}

The brain represents only $2 \%$ of the body mass, but consumes $>20 \%$ of $\mathrm{O}_{2}$ and glucose (Mink et al., 1981); the result of the high energy demand of neurons to maintain ion gradients (Attwell and Laughlin, 2001; Howarth et al., 2010; Harris and Attwell, 2012) and the almost exclusive use of mitochondrial oxidative phosphorylation for the production of ATP (Mergenthaler et al., 2013). As such, even transient reductions in $\mathrm{O}_{2}$ or glucose put neurons at risk of dysfunction and death.

During aging (Harman, 1972; Navarro et al., 2002), brain mitochondrial function is compromised, expressed as a reduction in oxidative phosphorylation (OxPhos), transport of mitochondria from soma to energy demanding synapses and excessive mitochondrial fission (Chaturvedi and Flint Beal, 2013). Thus normal aging sets the stage for limited mitochondrial response to ischemia. These mitochondrial defects could be responsible for the increase in the prevalence of stroke, increase in severity of infarcts and the limited recovery from stroke with advancing age. 
With age, the brain experiences a reduced capacity to take up and utilize glucose as an energy source (Mink et al., 1981). This reduction in glucose utilization in the face of persistent energy demand means that the brain must tap into other sources of energy to supply its basic functions and to respond to ischemic events. In the absence of glucose, brain mitochondria use ketone bodies derived from endogenous sources or dietary lipids through $\beta$-oxidation to supply carbon fragments for OxPhos (Cahill, 2006). Inasmuch normal aging compromises mitochondrial structure, function and movement, the possibility is strong that progressive mitochondrial defects contribute to the age-related increase in stroke prevalence, severity and lack of recovery.

Several neurological diseases such as multiple sclerosis, Parkinson's disease, and Alzheimer's disease have a common pathological finding of neuroinflammation (Di Filippo et al., 2010). Moreover, it is well accepted during the pathogenesis of both inflammatory and neurodegenerative CNS disorders mitochondria are crucial players. Because neurons are in high energy demand, and a slight decline in ATP production results in increased susceptibility to death leads to the hypothesis that mitochondrial dysfunction may be the link between neuroinflammation and neuronal degeneration. Indeed, mitochondria can be the switch for the initiation of apoptosis through the opening of mitochondrial permeability transition pores (mtPTP) resulting in the dissipation of the mitochondrial inner membrane potential. This dissipation of mitochondrial inner membrane potential causes the release of several molecules that activate caspases leading to apoptosis. Several cytokines, including TNF- $\alpha$, are known to affect mitochondrial function in non-neuronal cells; however, the cytokine-induced neuronal mitochondrial dysfunction still remains controversial. Although it is believed that an acute neuroinflammation may be beneficial to the CNS through preconditioning to prevent further 
damage to the neurons, chronic inflammation may represent a self-perpetuating detrimental response after an initial insult such as stroke.

Further, the extent to which factors known to increase risk of stroke do so by decreasing mitochondrial function is a challenge to the field of stroke research. Dysregulated inflammation is associated with many of the risk factors associated with stroke such as hypertension, diabetes and obesity (Smith et al., 2013). An elevated systemic inflammatory profile is associated with atherosclerosis, hypertension, diabetes, obesity, and peripheral infection. Thus far, studies have provided evidence that the release of oxidants, proteolytic enzymes and inflammatory cytokines alter blood brain barrier (BBB) permeability. The BBB excludes the majority of bacteria; however, similar to ischemia and reperfusion, inflammation induced by bacteria alters BBB permeability. Thus, understanding the effect of inflammation on mitochondrial function is important for increasing our understanding of how inflammation post-stroke can increase neurons susceptibility to death resulting in increased infarct size and poor outcome, and how inflammation plays a role in increasing the susceptibility to stroke. Finally, methods of enhancing mitochondrial functions as a therapeutic target to prevent, treat and recover from stroke have not yet been explored.

\subsection{Cytokine Changes Following Stroke}

Cytokines are soluble glycoproteins that are produced by cells in the brain in response to damaged tissue after ischemia and are responsible for regulating the innate and adaptive immune response (Ferrarese et al., 1999). Microglia, astrocytes, endothelial cells, and neurons in the brain are able to secrete pro-inflammatory and anti-inflammatory cytokines (Ferrarese et al., 1999). An increase in production of pro-inflammatory cytokines and a decrease in production of 
anti-inflammatory cytokines is correlated with a larger infarct size in animal models and a worse clinical outcome (Vila et al., 2003). TNF- $\alpha$, interleukin (IL)-1 $\beta$, IL-6, and IL-10 are inflammatory cytokines that have been found to be related to ischemic stroke and have been implicated as therapeutic targets and biomarkers for prognosis. The inflammatory response to ischemia changes with time, and knowing the timing of when these cytokines are increased or decreased and how these cytokines affect infarct volume is important in understanding how cytokines can be utilized clinically. TNF- $\alpha$ and IL-1 $\beta$ are well characterized in experimental stroke studies and human subjects. However, little is known about the temporal profile of IL-6 and IL-10, and fewer experimental stroke investigations have studied IL-6.

\section{A. TNF- $\alpha$}

Tumor necrosis factor-alpha (TNF- $\alpha$ ) precursor is membrane bound and cleaved by TNF- $\alpha$ converting enzyme (TACE) to release the soluble form that can bind to TNFR1 or TNFR2 (Liu et al., 1996). Most cells in the brain express TNFR1, and TNFR1 has an intracellular death domain (DD) with which adaptor proteins such as TNFR-associated DD protein (TRADD) interacts, and the interaction of TRADD with the intracellular DD of TNFR1 elicits signaling for cell death or cell survival by recruiting other adaptor proteins (Liu et al., 1996; Gourin and Shackford, 1997; Chan et al., 2000). The recruitment of adaptor proteins such as caspase-8 and FAS-associating protein with a death domain (FADD) result in apoptosis, and recruitment of TNF-receptor associating factor 2 (TRAF2) and receptor-interacting protein (RIP) activate NF$\kappa \mathrm{B}$ resulting in activation of genes for anti-inflammatory, anti-apoptotic proteins along with proinflammatory, apoptotic, and cytotoxic proteins (Gourin and Shackford, 1997). Therefore, the structure of the TNF-signaling complex enables TNF- $\alpha$ to induce inflammation and cell death after stroke or induce tolerance to ischemia. 


\section{$\underline{\text { Experimental Stroke Studies }}$}

Consequently in experimental stroke studies, it is not surprising that TNF- $\alpha$ has been shown to exacerbate infarct size and be neuroprotective. In a mouse distal permanent middle cerebral artery occlusion (pMCAO) electrocoagulation ischemic model, intracisternal injection of TNF- $\alpha$ 48 hours before occlusion decreases infarct volume (Nawashiro et al., 1997c). Furthermore, using the same ischemia model TNF- $\alpha$, TNFR1, and TNFR2 knock-out mice show larger infarct size compared to control mice suggesting that TNF- $\alpha$ is neuroprotective (Lambertsen et al., 2009). However, multiple approaches suggest that TNF- $\alpha$ is neurotoxic in stroke. I.p. or i.v. injection of TNF- $\alpha$ binding protein immediately after occlusion and topical administration of TNF- $\alpha$ binding protein immediately and 1 hour after occlusion in the distal pMCAO electrocoagulation ischemic model causes a dose-dependent decrease in infarct volume at 1 hour, 24 hours, and 2 weeks (Nawashiro et al., 1997a, b). Also, in the one hour distal transient middle cerebral artery occlusion (tMCAO) filament ischemia mouse model, i.c.v. injection of anti-TNF antibody decreases infarct size (Yang et al., 1999). In the rat ischemia model using the distal tMCAO and distal pMCAO where the vessel is occluded and cut, treating with TNF- $\alpha 24$ hours before occlusion is neurotoxic and increases infarct volume (Barone et al., 1997). Also, in the distal pMCAO thromboembolic model, inhibiting TACE decreases infarct volume. In agreement with the mouse model, administrating anti-TNF antibody and TNF- $\alpha$ binding protein in the rat ischemia model decreases infarct volume (Dawson et al., 1996; Lavine et al., 1998). The neurotoxic versus neuroprotective action of TNF- $\alpha$ is most likely due to signaling through nuclear factor $\mathrm{\kappa B}$ producing both pro-inflammatory and anti-inflammatory mediators and the timing of administration of TNF- $\alpha$ in experimental stroke models. 


\section{$\underline{\text { Time Course in Experimental Stroke Studies }}$}

After stroke onset, TNF- $\alpha$ mRNA expression is induced within thirty minutes after both tMCAO and pMCAO in mice and rats, and TNF- $\alpha$ protein expression is seen within 1 hour in the cortex and striatum after tMCAO in spontaneously hypertensive rats (Liu et al., 1994). Levels of TNF$\alpha$ mRNA and protein peak between 12 and 24 hours in both mice and rats and remain elevated up to 10 days in mice and 6 days in rats (Buttini et al., 1996; Gregersen et al., 2000). The timing of the expression of TNF- $\alpha$ after ischemia is found to be comparable among different mouse strains after pMCAO, but the level of expression is different (Lambertsen et al., 2002). Cells producing TNF- $\alpha$ are found at 12 and 24 hours after stroke in the penumbra of all mouse strains (Lambertsen et al., 2002). Interpretation of the time course of TNF- $\alpha$ is complicated in animal models because of the use of different strains of mice and rats, different methods for measuring levels of TNF- $\alpha$, and the time after stroke when levels are measured.

\section{Time Course in Human Studies}

In postmortem brain tissue studies, TNF- $\alpha$ positive cells are observed in all ischemic brains of severe patients 3 days post-stroke and are present up to 15 months post-stroke, and the majority of TNF- $\alpha$ positive cells are microglia and macrophages (Tarkowski et al., 1997; Dziewulska and Mossakowski, 2003). TNF- $\alpha$ positive neurons peak between 2 and 3 days post-stroke, TNF- $\alpha$ positive infiltrating immune cells from the periphery peak at day 3 , and TNF- $\alpha$ positive astrocytes peak from 15 hours to 14 days (Sairanen et al., 2001). Furthermore, TNF- $\alpha$ levels have been measured in CSF at $0,3,7,9,21,26$ days and 3 months after stroke, and TNF- $\alpha$ levels are only increased in stroke patients with white matter damage compared to normal controls at 3 months post-stroke (Tarkowski et al., 1997). However, other studies have shown that TNF- $\alpha$ levels are increased within 24 hours in the CSF (Vila et al., 2000a; Zaremba and 
Losy, 2001). Studies assessing TNF- $\alpha$ in serum of stroke patients have not been conclusive. A few studies found that serum TNF- $\alpha$ levels are not increased at admission or any time between 12 hours and 10 days after stroke (Fassbender et al., 1994; Montaner et al., 2003; Ormstad et al., 2011b). In contrast, other studies have shown that TNF- $\alpha$ is increased in the serum of stroke patients compared to controls within 6 hours and stay elevated for 10 days post-stroke (Zaremba and Losy, 2001; Intiso et al., 2003). Moreover, studies measuring TNF- $\alpha$ in plasma observed a significant increase of TNF- $\alpha$ in stroke patients at admission and within 24 hours post-stroke (Vila et al., 2000a; Castellanos et al., 2002). The time after stroke when levels of TNF- $\alpha$ are measured is critical and a contributing factor to the difference in results among and between animal and human studies. Additionally, the severity of stroke may be a key factor in the timing and magnitude of the increase in TNF- $\alpha$.

\section{B. IL1- $\beta$}

IL1- $\beta$ mRNA and protein is constitutively expressed in the brain at low levels and is regulated by transcription, translation, cleavage and cellular release. IL1- $\beta$ is synthesized as a large precursor protein and is biologically inactive until it is cleaved by caspase- 1 and secreted (Thornberry et al., 1992). Once it is biologically active, it binds to type 1 IL-1 receptor (IL-1R1) which associates with IL-1-receptor accessory protein (IL-1RAcP) that initiates intracellular signaling (Korherr et al., 1997). IL1- $\beta$ can also bind to IL-1R2, but IL-1R2 does not initiate downstream signaling because IL-1R2 does not have an intracellular-signaling domain (Thornberry et al., 1992; Korherr et al., 1997). Furthermore, IL-1R1 and IL-1R2 can exist in soluble forms when they are shed from cell membranes (Thornberry et al., 1992; Korherr et al., 1997; Allan et al., 2005). IL-1R2 and the soluble forms of both receptors act as decoy receptors that prevent a ligand from initiating signaling pathways (Allan et al., 2005). During acute 
ischemia, there is an increase in ATP which promotes the cleavage and cellular release of IL1- $\beta$ (Thornberry et al., 1992). An increase in IL1- $\beta$ can cause an increase in calcium entry through NMDA-receptor ion channels resulting in neuronal cell death (Viviani et al., 2003).

Furthermore, IL1- $\beta$ can potentiate inflammation by activating microglia, and IL1- $\beta$ increases leukocyte infiltration by increasing the expression of adhesion molecules on endothelial cells and causing breakdown of the blood brain barrier contributing to an increased infarct size and poor clinical outcome (Bernardes-Silva et al., 2001; Konsman et al., 2004; Mazzotta et al., 2004).

\section{Experimental Stroke Studies}

Numerous studies have shown IL1- $\beta$ to be neurotoxic in animal ischemia models. In the mouse distal electrocoagulation pMCAO model, injecting an IL-1 receptor antagonist immediately and 4 hours after stroke decreases infarct volume (Nawashiro et al., 1997b). Moreover in the proximal tMCAO filament model, treating with caspase-1 inhibitors 15 minutes before and immediately after stroke decreased infarct volume, and using caspase- 1 knock-out mice result in smaller infarct volume compared to control mice (Hara et al., 1997a; Hara et al., 1997b). IL1- $\alpha \beta$ knock-out mice also show smaller infarct volumes (Ohtaki et al., 2003). Similarly, the rat proximal electrocoagulation and filament pMCAO model exhibit an increase in infarct size in animals treated with IL1- $\beta 30$ minutes before and after stroke and a smaller infarct size, improved neurological score, and decreased number of leukocytes in the ischemic tissue when treated with an IL-1 receptor antagonist at the time of occlusion along with injections at 4, 8, 12, and 18 hours after stroke (Garcia et al., 1995; Loddick and Rothwell, 1996; Relton et al., 1996). Proximal tMCAO filament models further shows IL1- $\beta$ neurotoxicity, confirming what was seen in the rat pMCAO and mouse ischemia models. Caspase- 1 inhibitors decrease infarct volume in 
the tMCAO filament model when injected 15 minutes before surgery and after reperfusion, and injecting IL1- $\beta$ after reperfusion increases infarct size and brain edema (Yamasaki et al., 1995; Hara et al., 1997b). IL-1 $\beta$ is a potent pro-inflammatory cytokine and has a role in activation of numerous inflammatory processes. Using different ischemia models and different treatments to enhance or inhibit IL- $1 \beta$ has resulted in the observation that IL- $1 \beta$ is constantly neurotoxic in experimental stroke models.

\section{$\underline{\text { Time Course in Experimental Stroke Studies }}$}

IL1- $\beta$ positive microglia cells are found in the cortex 6 to 24 hours after stroke and elevated IL1$\beta$ mRNA levels are observed at 10 hours in the brain tissue in both the proximal pMCAO and proximal tMCAO filament mouse model (Hill et al., 1999; Clausen et al., 2008). However, levels of IL1- $\beta$ mRNA peak later at 18 hours in the pMCAO model compared to peak levels at 10 hours in the tMCAO model. In rat ischemia models, peak levels of IL1- $\beta$ mRNA are seen at 6 hours in the proximal tMCAO and distal pMCAO suture model (Wang et al., 1994; Berti et al., 2002) and elevated levels are seen as early as 1 hour after stroke (Liu et al., 1993; Wang et al., 1994). Thus, both rat and mouse ischemia models show an early increase in expression of IL-1 $\beta$ with peak expression dependent on the occlusion model used. Reperfusion allows for increased access of leukocytes to the brain which may contribute to levels of expression peaking earlier in the tMCAO model.

\section{$\underline{\text { Time Course in Human Studies }}$}

IL1- $\beta$ is elevated in the CSF of severe stroke patients with peak levels at 2-3 days post-stroke (Tarkowski et al., 1995, 1997). However another study showed, IL1- $\beta$ in CSF was elevated within 6 hours after stroke regardless of its severity (Beridze et al., 2011). Two peripheral blood 
studies found elevated levels of IL1- $\beta$ in plasma and serum of stroke patients (Mazzotta et al., 2004; Sotgiu et al., 2006). However, all other studies showed no increase of IL1- $\beta$ in serum or plasma (Fassbender et al., 1994; Tarkowski et al., 1995; Ormstad et al., 2011b). IL-1 $\beta$ has a highly localized role at the site of inflammation, and this localized role may be why IL-1 $\beta$ is not seen in plasma or serum of stroke patients. However, soluble IL-1 receptor decoys are elevated in plasma very early after stroke onset, which suggests an early attempt to regulate IL-1 $\beta$ supporting the important role IL-1 $\beta$ plays in stroke (Emsley et al., 2007).

\section{IL-6}

IL-6 is an inflammatory cytokine that binds to class I cytokine receptors (Erta et al., 2012).

Class I cytokine receptors do not have intrinsic enzyme activity; thus, IL-6 signaling through its class I cytokine receptor requires recruitment of an additional receptor protein gp130 (Saito et al., 1992). IL-6 signaling activates intracellular tyrosin-kinases such as Janus Kinase (JAK) which activates signal transducer and activator of transcription (STAT) family of transcription factors and RAS-RAF-MAPK pathways (Erta et al., 2012). These transcription factors and pathways can increase astrogliosis and angiogenesis which are important for tissue remodeling and recovery after stroke (Tancredi et al., 2000; Hakkoum et al., 2007). Furthermore, IL-6 can inhibit TNF- $\alpha$, induce apoptosis in neutrophils, and recruit monocytes and T-cells causing the transition between innate and adaptive immune response (Marz et al., 1996). However, IL-6 can be detrimental by increasing body temperature which has been shown to increase brain damage after stroke (Azzimondi et al., 1995). 


\section{Experimental Stroke Studies}

Few studies have been completed that assess IL-6 and stroke. IL-6 knock-out mice do not show a difference in infarct size compared to wild types mice in the proximal tMCAO filament model (Clark et al., 2000). However, when body temperature of IL-6 knock-out mice is controlled to levels of wild type mice, IL-6 knock-out mice exhibit larger infarcts suggesting IL-6 is neuroprotective (Herrmann et al., 2003). This finding is consistent with the proximal pMCAO electrocoagulation rat model in which IL-6 was injected 30 minutes before and 15 minutes after pMCAO and infarct size was reduced (Loddick et al., 1998).

\section{$\underline{\text { Time Course in Experimental Stroke Studies }}$}

IL-6 is elevated as early as 3-3.5 hours post-stroke and peaks between 6 and 24 hours depending on the ischemia model. In both mouse proximal pMCAO and tMCAO filament model, levels of IL-6 mRNA are elevated at 10 hours and peak expression is at 18 hours. In rat proximal tMCAO models, elevated IL-6 mRNA and protein levels are seen 3-3.5 hours after stroke and peak expression of IL-6 protein at 12 and 24 hours (Wang et al., 1995; Suzuki et al., 1999).

\section{Time Course in Human Studies}

CSF IL-6 is elevated in stroke patients and increases within 24 hours and peaks 2-3 days after stroke (Tarkowski et al., 1995, 1997). Vila et al. (2000a) and Beridze et al. (2011) found that CSF IL-6 levels are elevated but only in severe stroke patients. Furthermore, IL-6 is elevated in serum and plasma during the first week after stroke in all published studies. However, some studies show IL-6 levels peaking at 10 hours in serum while other studies show levels of IL-6 peaking between 3 and 7 days after stroke (Fassbender et al., 1994; Perini et al., 2001; WajeAndreassen et al., 2005). Thus, there is agreement that IL-6 is elevated in stroke patients during 
the week after stroke onset. However, the timing at which IL-6 levels peak appear to depend on stroke severity and stroke type.

\section{IL-10}

IL-10 is an anti-inflammatory cytokine that signals through the IL-10 receptor complex, composed of two chains of IL-10R1 and two chains of IL-10R2 (Krause et al., 2002). The two chains of IL-10R1 bind IL-10 and IL-10R2 initiates signal transduction. Jak1 is constitutively bound to IL-10R1 and Tyk2 is constitutively bound to IL-10R2, and when the IL-10 receptor complex is activated by IL-10, two tyrosine residues on the intracellular domain of IL-10R1 are phosphorylated by cross-phosphorylation and Jak 1 and Tyk2 become activated (Kotenko et al., 1996). Stat3 interacts with these phosphorylated tyrosines to phosphorylate other Stat proteins that translocate to the nucleus to activate transcription of Stat3-responsive genes (Finbloom and Winestock, 1995). IL-10 signaling blocks pro-inflammatory cytokine production, chemokine secretion, and inhibits antigen presentation by macrophages and microglia (Ooboshi et al., 2005). Furthermore, IL-10 can counteract the detrimental effects of TNF- $\alpha$ by inhibiting activation of signaling pathways induced by TNF- $\alpha$ (Liesz et al., 2009). IL-10's beneficial antiinflammatory effects are an attractive target for potential clinical applications in stroke.

\section{$\underline{\text { Experimental Stroke Studies }}$}

Administration of IL-10 into the lateral ventricle 30 minutes and 3 hours after stroke in the pMCAO electrocoagulation rat model significantly decreases infarct size (Spera et al., 1998). In the same model, infarct size is significantly decreased when IL-10 is administered 30 minutes post stroke for 3 hours systemically into the tail vein (Spera et al., 1998). Furthermore, injecting adenoviral vectors encoding IL-10 into the lateral ventricle 60-90 minutes before photochemical 
occlusion or bilateral carotid occlusion in rats decreases infarct size and infiltrating leukocytes (Ooboshi et al., 2005). In a mouse transgenic model in which astrocytes, microglia, and endothelial brain cells overexpress IL-10, smaller infarcts are observed, and IL-10 deficient mice show larger infarcts after pMCAO (Grilli et al., 2000; de Bilbao et al., 2009). Another study suggesting the beneficial role of IL-10 in stroke, found a subset of regulatory B-cells that secrets IL-10 can decrease infarct size and improve neurological score (Fillatreau et al., 2002; Mann et al., 2007; Ren et al., 2011). Collectively, all experimental stroke studies suggest that IL-10 is neuroprotective through suppression of the inflammatory response after ischemia.

\section{$\underline{\text { Time Course in Experimental Stroke Studies }}$}

IL-10 mRNA expression is increased in the ipsilateral cortex of the pMCAO mouse model at 6 hours and peaks at 7 days, and IL-10 receptor mRNA is upregulated after pMCAO (Li et al., 2001; Perez-de Puig et al., 2013). At 1, 4, and 7 days after pMCAO, IL-10 receptor positive cells are found in the subarachnoid space and on the surface of the infarcted cortex, and IL-10 receptor positive astrocytes are found in the infarcted tissue at day 4 (Perez-de Puig et al., 2013). Furthermore in the rat ischemia model, IL-10 is decreased 12 hours after stroke but increases six-fold two days post-stroke (Li et al., 2001). Few studies have examined the temporal profile of IL-10 in experimental stroke models, and these studies measured IL-10 mRNA expression in the brain not in the periphery.

\section{$\underline{\text { Time Course in Human Studies }}$}

More studies have assessed the temporal profile of IL-10 in human subjects. Stroke patients show lower levels of IL-10 in plasma within 12 hours after stroke compared to controls, and 24 hours after tPA treatment IL-10 levels are increased (Perini et al., 2001; Mazzotta et al., 2004). 
Furthermore, levels of IL-10 are decreased within 24 hours of stroke and levels increase over 72 hours post-stroke (Nayak et al., 2012). Studies report a huge variability in the levels of IL-10, which is most likely due to the time the sample was taken and stroke severity. Furthermore, the heterogeneity of co-morbidities among stroke patients could play a role in the variability in levels of IL-10 and other cytokines after stroke.

\subsection{Effects of Cytokines on Stroke Outcome}

The inflammatory response to ischemia plays an essential role in the pathophysiology of stroke, and using inflammatory markers such as the cytokines described above can be useful to predict outcome after stroke. Increased mortality and functional disability occurs in stroke patients who show neurological symptoms that progress acutely after stroke onset. The ischemic penumbra is the tissue that surrounds the infarct core and can survive or be transformed into necrotic tissue and become part of the infarcted tissue due to reduced blood flow and impaired neuronal function (Astrup et al., 1981). The transformation of the penumbra to infarcted tissue results in worsening of neurological symptoms (del Zoppo et al., 2011). Increased levels of IL-6 in both CSF and serum have been associated with worsening of neurological symptoms, increased infarct size and poor functional outcome (Tarkowski et al., 1997; Vila et al., 2000a; Beridze et al., 2011). It is surprising that IL-6 is associated with poor functional outcome in humans since in experimental stroke studies IL-6 was found to be neuroprotective. However, Ormstad et al. (2011b) found no correlation between IL-6 and infarct size, and Sotgiu et al. (2006) found an inverse correlation of IL-6 with infarct size and poor outcome. IL-6 can cause the release of prostaglandin E2 in the brain, and prostaglandin E2 acts on the hypothalamus resulting in an increase in body temperature. Numerous human and experimental stroke studies have shown that fever is correlated with increased infarct size and poor outcome; thus, the early increase in 
IL-6 and prolonged elevation of IL-6 in the CSF and blood most likely correlates with increased risk of elevated temperature that increases inflammation and tissue damage after stroke. Moreover, there are conflicting findings when using TNF- $\alpha$ as an inflammatory mediator for predicting outcome and infarct size. Increased levels of TNF- $\alpha$ in the serum and CSF of stroke patients is correlated with worsening of neurological symptoms, increased infarct size and poor outcome at 3 months in studies including severe strokes and patients with white matter damage (Tarkowski et al., 1995; Zaremba and Losy, 2001; Castellanos et al., 2002; Montaner et al., 2003; Mazzotta et al., 2004). However, in other studies TNF- $\alpha$ was not correlated with outcome or infarct size (Vila et al., 2000a; Intiso et al., 2003). Although IL-1 $\beta$ was neurotoxic in all experimental ischemic animal studies, IL-1 $\beta$ was only found to be correlated with poor outcome in the plasma of patients in one study which is most likely due to the localized role of IL-1 $\beta$ and low levels of IL-1 $\beta$ in the blood or CSF (Mazzotta et al., 2004). The anti-inflammatory effects of IL-10 play an important role in preventing neuronal death; thus, it is not surprising that low levels of IL-10 early after stroke onset is correlated with poor outcome and increased infarct size (Mazzotta et al., 2004). However, a trend toward increased levels of IL-10 days after stroke and even at admission correlate with poor outcome and an increased risk for infection (Chamorro et al., 2006). Increased IL-10 down regulates TNF- $\alpha$ and inhibits IFN- $\gamma$ production which can be beneficial in the earlier phases of infarct progression after stroke; however, the increase in IL-10 can induce peripheral immunosuppression resulting in post-stroke infection and infections are the leading cause of death in stroke patients (Zhang et al., 2010).

The variability of the data on peripheral cytokines in human stroke is most likely due to the heterogeneity of stroke in humans. Stroke severity, stroke location, age, co-morbidities, and systemic inflammation prior to stroke may be key factors contributing to the levels of peripheral 
cytokines seen post-stroke. Furthermore, proper control subjects are important for interpreting cytokine data. In human stroke studies assessing IL-10, TNF- $\alpha$, IL-1 $\beta$, IL-6, and other inflammatory mediators, the majority of the studies had age-matched controls but some studies had healthy controls subjects while other studies had controls with co-morbidities such as hypertension or diabetes. In addition, other studies compared cytokine levels based on stroke severity. Thus, it is important to control for co-morbidities and risk factors to determine which and when cytokines are increased due to stroke. Consequently, the use of only one inflammatory mediator to predict outcome or infarct size is not clinically useful due to the heterogeneity of the peripheral cytokine response. Therefore, further understanding of when these cytokines are increased and how they interact with each other can help elucidate how these cytokines can be used clinically as biomarkers to help predict outcome.

I assessed the effects of these 4 cytokines on neuronal mitochondrial function with the hypothesis that pro-inflammatory cytokines (TNF- $\alpha$ and IL-1 $\beta$ ) would decrease mitochondrial function resulting in neurotoxicity and anti-inflammatory cytokines (IL-6 and IL-10) would increase mitochondrial function resulting in neuroprotection. I found that exposure to 1000 $\mathrm{ng} / \mathrm{ml}$ IL-1 $\beta$ for 12 hours significantly decreased neuronal ATP production and maximal respiration (Figure 1.1) without causing cell death (data not shown). 


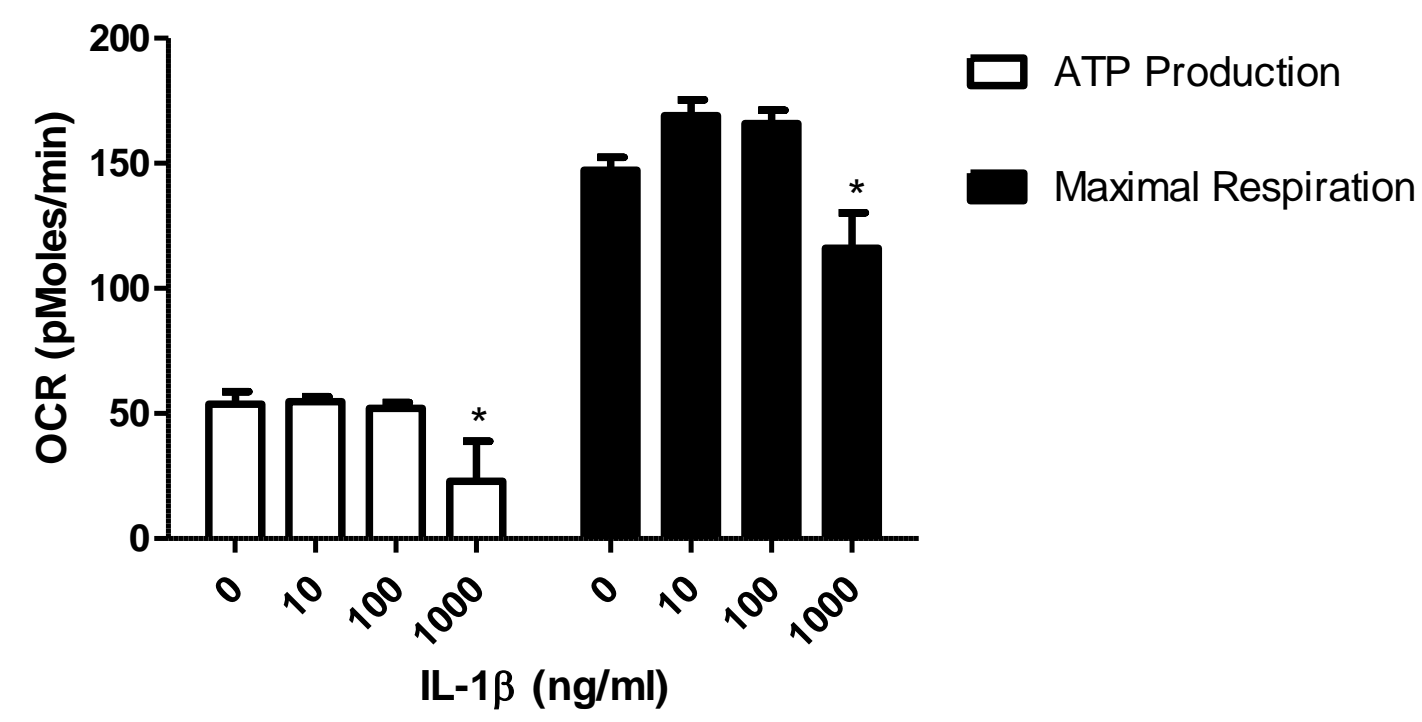

Figure 1.1: ATP production and Maximal respiration after 12 hour exposure to IL-1 $\beta . * \mathrm{p}<0.05$

IL-6 (Figure 1.2) and IL-10 (Figure 1.3) both increased neuronal mitochondrial function after 6 hours of exposure; however, TNF- $\alpha$ had the most profound dose-dependent effect on neuronal mitochondrial function; thus, we decided to further investigate the mechanism by which TNF- $\alpha$ affected mitochondrial function. 


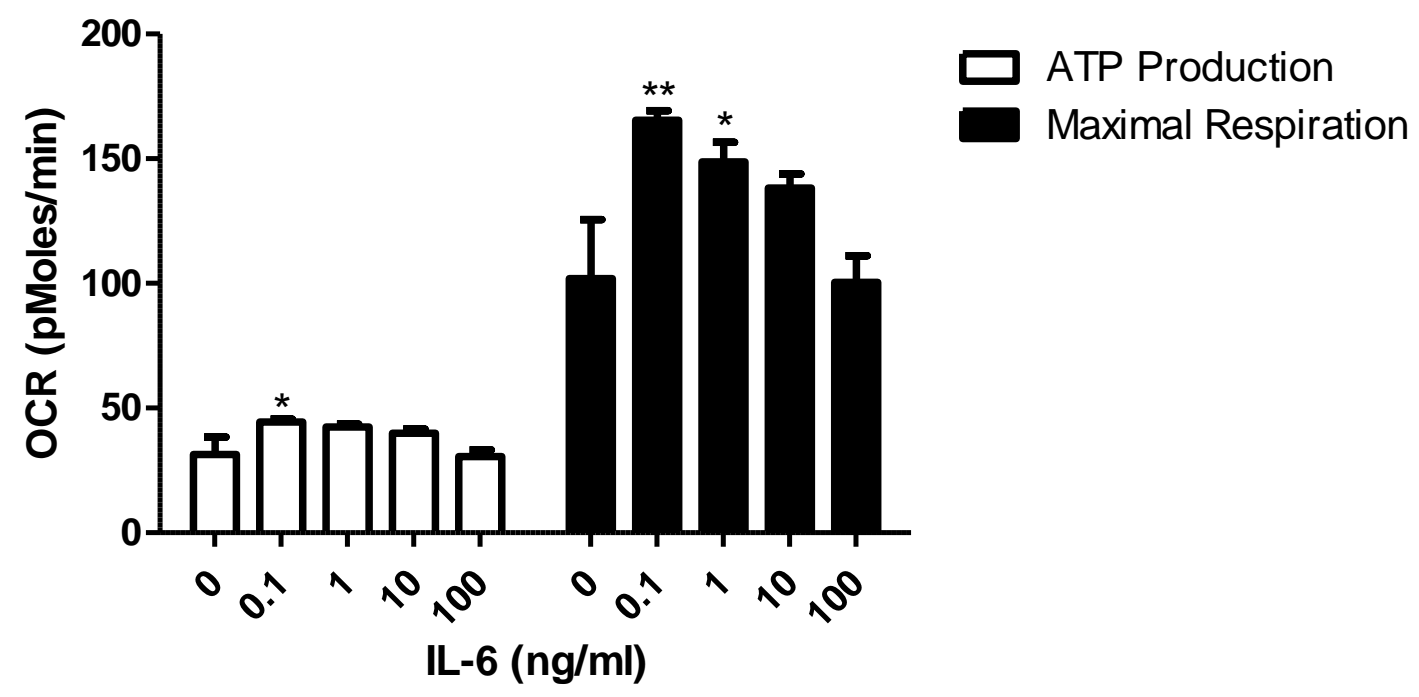

Figure 1.2: ATP production and Maximal respiration after 6 hour exposure to IL-6. ${ }^{*} \mathrm{p}<0.05$; $* * \mathrm{p}<0.01$

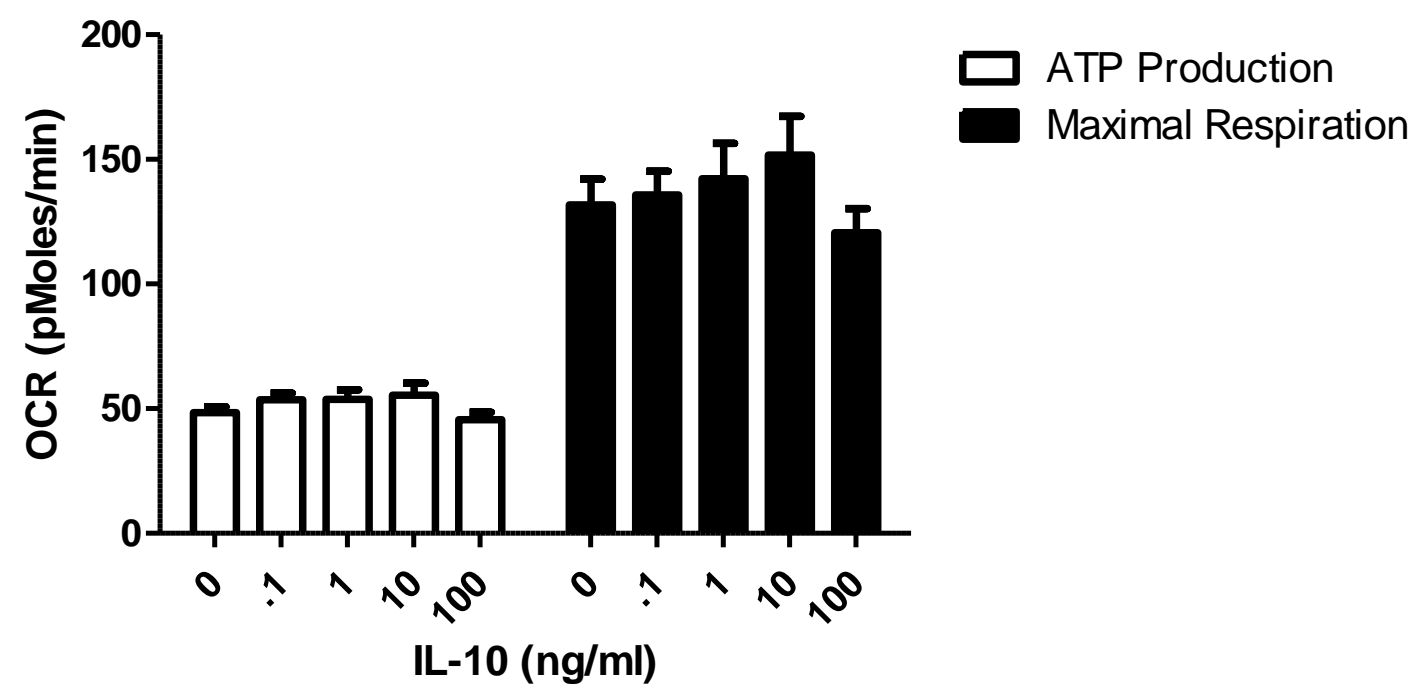

Figure 1.3: ATP production and maximal respiration after 6 hour exposure to IL-10.

\subsection{Gaps in Knowledge}

Since cytokines play an essential role in stroke and have potential clinical utility, it is critical to understand how experimental and human studies contribute to our knowledge on how to 
effectively use cytokines clinically and how experimental and human studies can be improved to increase the likelihood of clinically translatable results. An important confounding factor in understanding the role of peripheral cytokines in human stroke is that experimental stroke studies observe the cytokine response to stroke in the brain not in the periphery. Furthermore, in experimental stroke studies both permanent and transient MCAO models are invasive and could contribute to the cytokine response especially in stroke models that involves craniectomy. Transient MCAO mimics occlusion and reperfusion while pMCAO mimics just occlusion. Although reperfusion can attenuate infarction, it allows for increased infiltration of leukocytes into the brain, and the contribution of cytokines from peripheral leukocytes in affecting the progression of infarction and the difference in kinetics of leukocytes in the two ischemia models is not well studied. Thus, further understanding of how reperfusion can affect cytokine levels and their impact on outcome will be beneficial clinically in patients that receive tPA. Although outlined above is the signaling mechanisms for TNF- $\alpha$, few studies have assessed mechanisms of TNF- $\alpha$ or any other cytokines in stroke, and there is a substantial lack in understanding of how cytokines balance their neuroprotective and neurotoxic action after stroke. Understanding this balance is crucial for defining a therapeutic time window for using cytokines as biomarkers or therapeutic targets.

Understanding the effects of different experimental stroke models on cytokine expression is important, but equally important is understanding how location of stroke, age, co-morbid diseases and environmental factors affect cytokine expression in human stroke studies. Thus, using clinically relevant stroke models such as aged animals, animals with hypertension, diabetes, etc., and producing strokes in different locations will provide essential information on cytokine expression that can be more easily translated from the bench to the bedside. 
Additionally, few human stroke studies assess the peripheral cytokine response before 4 hours after stroke which is a critical time in infarct progression, and early inflammatory events have been show to play a role in recovery from stroke. Thus, understanding these early cytokines responses and their effects early post-stroke is critical in using cytokines as biomarkers for prognosis and therapeutic targets. Designing clinical stroke studies to have power to do analysis on cytokine expression controlling for time, location, and co-morbidities and designing preclinical stroke studies to assess cytokine expression in peripheral blood as well as brain will be critical for the use of cytokines as prognostic biomarkers for stroke.

While prognostic biomarkers are important clinically, it is equally as important to find new therapeutic targets for stroke. Currently there is only one FDA approved drug for stroke, tPA, that less than 5\% percent of patients receive. Thus, understanding the mechanism by which cytokines such as TNF- $\alpha$ exacerbate stroke damage and the expression of these cytokines pre and post-stroke is critical for the advancement of stroke research.

\subsection{Summary}

It is clear that cytokines play an important role in the pathophysiology and etiology of stroke, and the loss in balance between pro-inflammatory and anti-inflammatory cytokines affects infarct size and functional outcome. Thus, focusing on one cytokine only as a potential biomarker or a therapeutic target likely will not be advantageous in stroke. Future work needs to elucidate the temporal profile of cytokines in the periphery in human and experimental stroke studies to determine which cells contribute to the elevation of cytokines in the brain and blood and to understand how they work in concert to provide neuroprotection or increase neurotoxicity. The crosstalk between the immune system and the brain is still not well understood. Using cytokines as biomarkers or therapeutic targets may be beneficial to 
understand the post-ischemic immune response and its effects on outcome clinically and to modulate the post-ischemic immune response to limit tissue damage. However, modulation of the immune response can also be detrimental after stroke; thus, it is imperative that further clinical and experimental studies be pursued to better understand the complex interaction between the immune system and the brain after stroke. Moreover, understanding how having a stroke during an active infection or a pro-inflammatory state contributes to the long-term deficits post-stroke is essential for future treatment of stroke. Numerous risk factors such as diabetes, hypertension, and atherosclerosis have been recognized to increase susceptibility to stroke; however, the epidemiology of ischemic stroke is not sufficiently explained by the prevalence of these cerebrovascular risk factors (Grau et al., 1995b). In at least a third of ischemic stroke cases, the patients lack traditional risk factors and have no apparent cause. The presence of systemic infection could be an important mediating risk factor. Indeed, thirty to forty percent of strokes occur during or acutely after an infection (Grau et al., 1995b) suggest an association between systemic infection and stroke. Recent studies have indicated that both Helicobacter pylori (H. pylori) (Wang et al., 2012) and human cytomegalovirus (HCMV) (Huang et al., 2012) infections are associated with increased risk for ischemic stroke. Moreover, having a respiratory tract infection increases one's risk for an atherothrombotic ischemic event for up to 3 months (Zurru et al., 2009) and chronic bronchitis increases risk for stroke and transient ischemic attacks (TIA) (Grau et al., 2009). Lastly, Grabska et al. (2011) conducted a retrospective chart review of 2066 ischemic stroke patients to assess the effect of pre-stroke and post-stroke infection on ischemic stroke severity and found that pre-stroke infection increased poor outcomes within the first 30 days but did not have effects at 90 days on modified Rankin scores. Thus whether it is traditional stroke risk factors or infection that increases susceptibility to stroke, a key feature is an increased pro-inflammatory profile. McColl et al. (2007) found that 
the combination of a minor bacterial infection mimic, lipopolysaccharide (LPS) prior to a minor stroke exacerbates infarct volume in a mouse model. Thus, experimental and epidemiological data strongly suggest that infection and/or inflammation play a role in stroke occurrence and severity. However, to date, long-term outcomes of stroke during an active infection has not been studied.

Thus, my dissertation will use post-stroke relevant concentrations of TNF- $\alpha$ and clinically relevant exposure times to understand 1) if TNF- $\alpha$ affects neuronal mitochondrial function and 2) if TNF- $\alpha$ affects neuronal mitochondrial function, the mechanism by which it occurs. Additionally, my dissertation will focus on 3) if a bacterial infection mimic, lipopolysaccharide (LPS), prior to stroke affects stroke outcome. 


\section{Chapter 2}

\section{Rapid mitochondrial dysfunction mediates TNF-alpha induced neurotoxicity}

Danielle N. Doll, Stephanie L. Rellick, Taura L. Barr, Xuefang Ren, and James W. Simpkins

Doll DN, Rellick SL, Barr TL, Ren X, Simpkins, JW. (2015) Rapid mitochondrial dysfunction mediates TNF-alpha induced neurotoxicity. Journal of Neurochemistry 132, 443 - 451. 


\subsection{Abstract}

Tumor necrosis factor alpha (TNF- $\alpha)$ is known to exacerbate ischemic brain injury; however, the mechanism is unknown. Previous studies have evaluated the effects of TNF- $\alpha$ on neurons with long exposures to high doses of TNF- $\alpha$, which is not pathophysiologically relevant. We characterized the rapid effects of TNF- $\alpha$ on basal respiration, ATP production, and maximal respiration using pathophysiologically relevant, post-stroke concentrations of TNF- $\alpha$. We observed a reduction in mitochondrial function as early as 1.5 hours after exposure to low doses of TNF- $\alpha$, followed by a decrease in cell viability in HT-22 cells and primary neurons. Subsequently, we used the HT-22 cell line to determine the mechanism by which TNF- $\alpha$ causes a rapid and profound reduction in mitochondrial function. Pre-treating with TNF-R1 antibody, but not TNF-R2 antibody, ameliorated the neurotoxic effects of TNF- $\alpha$ indicating TNF- $\alpha$ exerts its neurotoxic effects through TNF-R1. We observed an increase in caspase 8 activity and a decrease in mitochondrial membrane potential after exposure to TNF- $\alpha$ which resulted in a release of cytochrome $c$ from the mitochondrial into the cytosol. These novel findings indicate for the first time that an acute exposure to pathophysiologically relevant concentrations of TNF$\alpha$ has neurotoxic effects mediated by a rapid impairment of mitochondrial function. 


\subsection{Introduction}

Inflammatory mechanisms play a crucial role in the pathophysiologic processes after the onset of ischemic stroke (Vila et al., 2000a). Ischemic brain injury is complex, and intracellular signaling that regulates innate and adaptive immunity, inflammation, cell death, angiogenesis, and repair processes plays an important role in the initiation, progression, and resolution of ischemia. Initiation of these critical intracellular regulators occurs through rapid cytokine signaling after ischemia, highlighting cytokines as key regulators of stroke damage (Hallenbeck, 2002). TNF- $\alpha$ is one of many pro-inflammatory cytokines associated with worsened clinical outcomes after stroke and exacerbations of infarct size in pre-clinical models (Nawashiro et al., 1997c; Ormstad et al., 2011a). However, our understanding of the pro-inflammatory effects of TNF- $\alpha$ is based primarily on studies of its peripheral actions.

TNF- $\alpha$ mRNA increases within 1 hour in the ischemic injury core, and the expression of its immunoreactive protein increases within 2-6 hours after the onset of ischemia in pre-clinical models (Botchkina et al., 1997). TNF- $\alpha$ is increased in the serum of stroke patients between 6 and 12 hours after symptom onset (Liu et al., 1994; Sotgiu et al., 2006). TNF- $\alpha$ signaling occurs through the TNF- $\alpha$ receptor (TNF-R), where ligand binding recruits adaptor proteins to a core signaling complex allowing for differences in signal transduction depending upon the stimulatory pattern (Wallach et al., 2002).

The response to TNF- $\alpha$ is cell-type dependent. In bovine and rat oligodendrocytes, TNF- $\alpha$ induces apoptosis, while in astrocytes, TNF- $\alpha$ enhances major histocompatibility complex (MHC) class II and intracellular adhesion molecule 1 (ICAM-1) expression (Chao et al., 1995). In primary septo-hippocampal cultures, exposure to $30 \mathrm{ng} / \mathrm{ml}$ of TNF- $\alpha$ caused significant 
cytotoxicity but only with the addition of Actinomycin-D (Zhao et al., 2001). When rat primary cortical neurons are exposed to $10 \mathrm{ng} / \mathrm{ml}$ of TNF- $\alpha$ for 24 hours, neurite retraction or formation of apoptotic bodies were not observed (Reimann-Philipp et al., 2001). However, in PC12 cells, TNF- $\alpha$ was cytotoxic (Reimann-Philipp et al., 2001). While these studies show neurotoxic effects of TNF- $\alpha$, cultured embryonic rat hippocampal, septal, and cortical neurons are protected from glucose deprivation-induced injury and excitatory amino acid toxicity by exposure to TNF$\alpha$ (Cheng et al., 1994). Furthermore, TNF- $\alpha$ can induce the expression of anti-apoptotic proteins B-cell lymphoma 2 (Bcl-2) and B-cell lymphoma-extra large (Bcl-xl) in hippocampal neurons (Tamatani et al., 1999). These few studies used high concentrations and long periods of exposure to TNF- $\alpha$. Also, there is a single study, of adipocytes that used high concentrations and 4 day exposures to assess mitochondrial function (Chen et al., 2010).

In the present study, we tested the hypothesis that acute exposure to TNF- $\alpha$ concentrations seen in serum of stroke patients (Lambertsen et al., 2012; Nayak et al., 2012) causes neuronal cell death through rapid mitochondrial dysfunction. As such, we characterized the effect of acute exposure to low doses of TNF- $\alpha$ in a mouse hippocampal neuronal cell line (HT-22) and mouse primary cortical neurons. A rapid and profound mitochondrial dysfunction was observed after as little as 1.5 hours of exposure to TNF- $\alpha$ which preceded cell death. These findings suggest that the damaging effect of TNF- $\alpha$ may be due to the impairment of neuronal mitochondrial function. 


\subsection{Materials and Methods}

\section{Cell Culture}

An immortalized mouse hippocampal cell line, HT-22, was cultured in Hyclone DMEM/high glucose (Fisher Scientific, Waltham, MA) with 10\% fetal bovine serum (FBS) (Atlanta Biologicals, Flowery Branch, GA) and 1\% penicillin/streptomycin (Fisher Scientific). The HT22 mouse hippocampal neuronal cell line was provided by The Salk Institute for Biological Research (La Jolla, CA), and cell passages 15 - 23 were used. When HT-22 cells were at least $80 \%$ confluent, the cells were trypsinized and spun down at 1500 RPM for 3 minutes. The cells were counted with Nexcelom Bioscience Cellometer AutoT4 (Lawrence, MA). Primary mouse cortical neurons were purchased from Life Technologies (Carlsbad, CA) and cultured in Neurobasal ${ }^{\circledR}$ Medium supplemented with GlutaMAX ${ }^{\mathrm{TM}}-\mathrm{I}$ and B-27 ${ }^{\circledR}$ supplement (Life Technologies).

\section{TNF- $\alpha$ Treatment}

Recombinant Mouse TNF- $\alpha$ was purchased from R\&D Systems (Minneapolis, MN) and reconstituted at $50 \mu \mathrm{g} / \mathrm{ml}$ in phosphate buffered saline (PBS) containing $0.1 \%$ bovine serum albumin (BSA). Dilutions were made in Hyclone DMEM/high glucose with $10 \%$ FBS and $1 \%$ penicillin/streptomycin to obtain concentrations of $1,10,100$, and $1000 \mathrm{pg} / \mathrm{ml}$. The media was removed from each well by gentle aspiration. After removing the media, $100 \mathrm{ul}$ of Hyclone DMEM/high glucose or the various concentrations of TNF- $\alpha$ were added for either $1.5,3,6$, or 12 hours. These time points were chosen based upon the post-stroke increase in TNF- $\alpha$ in serum patients and in the brains of animal models (Liu et al., 1994; Botchkina et al., 1997; Zaremba and Losy, 2001; Intiso et al., 2003). For primary mouse cortical neurons, TNF- $\alpha$ at concentrations of 0,100 and $1000 \mathrm{pg} / \mathrm{ml}$ were added for $1.5,3,6$ and 24 hours. 


\section{Mitochondrial Function Assessment}

15,000 HT-22 cells or 16,000 primary mouse cortical neurons were seeded in a XF 96 cell culture microplate. HT-22 cells were treated with $1-1000 \mathrm{pg} / \mathrm{ml}$ of TNF- $\alpha$ ( 6 replicates per treatment condition) 24 hours after seeding. Primary mouse cortical neurons were treated with 100 or $1000 \mathrm{pg} / \mathrm{ml}$ of TNF- $\alpha 3$ days after seeding. After treating with TNF- $\alpha$, mitochondrial function was assessed with the $\mathrm{XF}^{\mathrm{e}} 96$ Analyzer (Seahorse Bioscience, North Billerica, MA) using a Mito Stress test kit at 1.5, 3, 6, and 12 hours (HT-22 cells) or 1.5, 3, 6, and 24 hours (primary neurons). Non-mitochondria derived oxygen consumption rate (OCR) was measured using the first measurement after addition of rotenone and antimycin a. To calculate basal respiration, the measurement prior to oligomycin addition was subtracted from nonmitochondria derived OCR. Proton leak was measured using the third measurement after oligomycin injection subtracted from non-mitochondrial derived OCR. ATP production was measured from subtracting proton leak from basal respiration. Maximal respiration was calculated using the first measurement after FCCP injection subtracted from non-mitochondria derived OCR.

\section{Cell Viability}

Cell viability was assessed using Calcein AM (Life Technologies), and reconstituted at $2 \mathrm{mM}$ in DMSO. 15,000 HT-22 cells or 16,000 primary mouse cortical neurons were seeded in a blackwalled clear bottom 96 well plate. HT-22 cells were exposed to $1-1000 \mathrm{pg} / \mathrm{ml}$ of TNF- $\alpha$ and the primary mouse cortical neurons were treated with 100 or $1000 \mathrm{pg} / \mathrm{ml}$ of TNF- $\alpha$. After exposure to TNF- $\alpha$ for $1.5,3,6$, and 12 hours (HT-22 cells) or 1.5, 3, 6, and 24 hours (primary neurons), the plate was washed three times with PBS 1X. $100 \mathrm{ul}$ of 1uM Calcein AM was added 
to the wells. The plate was incubated at room temperature in the dark for 30 minutes. The plate was read using a BioTek Synergy H1 Hybrid reader (Winooski, VT).

\section{Flow Cytometry}

HT-22 cells were grown to $90 \%$ confluence, a single cell suspension was made using cell dissociation buffer, and the cells were fixed in 10\% formaldehyde for 30 minutes and subsequently permeabilized in $70 \%$ ethanol for 30 minutes on ice. To reduce non-specific antibody binding, HT-22 cells were blocked in 3\% BSA in PBS for 15 minutes and subsequently incubated with 1ug PE anti-mouse CD120a (TNF R Type I/p55) or PE anti-mouse CD120b (TNF R Type II/p75) from Biolegend (San Diego, CA). Data were acquired by counting 10,000 events and analyzed using FACSCalibur (BD Biosciences, San Jose, CA) to determine the presence or absence of TNF-R1 and/or TNF-R2.

\section{TNF-R1 or TNF-R2 antibody Treatment}

HT-22 cells were pre-treated for 1 hour with 4, 6, or $8 \mathrm{ug} / \mathrm{ml}$ of LEAF ${ }^{\mathrm{LM}}$ Purified anti-mouse CD120a (TNF R Type I/p55), 4, 6, or 8 ug/ml of LEAF $^{\mathrm{LM}}$ Purified anti-mouse CD120b (TNF R Type II/p75), or 8 ug/ml of LEAF ${ }^{\mathrm{LM}}$ Purified Armenian Hamster IgG Isotype Ctrl from Biolegend. Media was removed and cells were then treated with $100 \mathrm{pg} / \mathrm{ml}$ of TNF- $\alpha$ for 1.5 hours or 3 hours. Cell viability was assessed with Calcein AM at both 1.5 and 3 hours, and mitochondrial basal respiration was assessed with the $\mathrm{XF}^{\mathrm{e}} 96$ Analyzer from Seahorse Bioscience at 1.5 hours. 
Caspase 8 and Caspase 3/7 Activity

Caspase 8 and Caspase $3 / 7$ activity was assessed with the Caspase-Glo ${ }^{\text {TM }} 8$ and Caspase-Glo ${ }^{\mathrm{TM}}$ 3/7 assay kits (Promega, Madison, WI) after treating HT-22 cells with 1, 10, 100, $1000 \mathrm{pg} / \mathrm{ml}$ of TNF- $\alpha$ for 1.5 hours.

\section{TMRE Mitochondrial Membrane Potential Assay}

Mitochondrial membrane potential was assayed using a TMRE mitochondrial membrane potential assay (Abcam,Cambridge, MA) by addition of $300 \mathrm{nM}$ TMRE and $20 \mu \mathrm{M}$ FCCP after treating HT-22 cells with 1, 10, 100, $1000 \mathrm{pg} / \mathrm{ml}$ of TNF- $\alpha$ for 1.5 hours.

\section{Cytochrome c release from mitochondria to cytosol}

After exposure to 100 or $1000 \mathrm{pg} / \mathrm{ml}$ of TNF- $\alpha$ for 3, 6, 12, and 24 hours, HT-22 cells were fractioned into cytosolic and mitochondrial fractions using the Mitochondrial/Cytosol Fractionation Kit (Biovision, Milpitas, CA). The protein concentration of each fraction was determined using the Pierce 660nm protein assay (Thermo Scientific, Waltham, MA). Cytochrome $c$ levels in the cytosolic and mitochondrial fractions were determined using a rat/mouse cytochrome $c$ Quantikine ELISA (R\&D Systems, Minneapolis, MN). Percentage release of cytochrome $c$ was calculated using the following equation: total cytosolic cytochrome c/ (cytosolic + mitochondrial cytochrome c) (Tanaka et al., 2004).

\section{Statistical Analysis}

One-way ANOVA and post hoc analyses (Dunnett or Trend for linear analysis) were used to analyze differences among groups using GraphPad Prism 5 software. Statistical significance was determined at a $\mathrm{p}<0.05$. 


\subsection{Results}

TNF- $\alpha$ caused a decrease in mitochondrial function at 1.5, 3, 6, and 12 hours in HT-22 cells

Figure 2.1 (A, C, E, G) and Supplemental Figure 1 show the dose-dependent decrease in basal respiration, ATP production, and maximal respiration at 1.5, 3, 6, and 12 hours of TNF- $\alpha$ exposure. At 1.5 hours, basal respiration, ATP production, and maximal respiration decreased by approximately $30 \%$, and $40 \%$ at 100 and $1000 \mathrm{pg} / \mathrm{ml}$ TNF- $\alpha$, respectively (Figure $2.1 \mathrm{~A}$ and Supplemental Figure 2.1A). At 3 and 12 hours, but not 6 hours, the magnitude of the dosedependent reduction in basal respiration, ATP production, and maximal respiration induced by TNF- $\alpha$ was increased (Figure 2.1 C, E, G and Supplemental Figure B - D).

TNF- $\alpha$ caused a delayed decrease in cell viability in HT-22 cells

Based on our observation of decreased mitochondrial function as early as 1.5 hours of TNF- $\alpha$ exposure, we determined if the decrease in mitochondrial function preceded cell death. We did not observe a significant decrease in cell viability after 1.5 hours of exposure to TNF- $\alpha$ (Figure 2.1 B). However, cell viability began to decrease after 3 hours by approximately $30 \%$ at doses of 100 and $1000 \mathrm{pg} / \mathrm{ml}$ of TNF- $\alpha$ (Figure 2.1 D). Additionally, cell viability decreased significantly at 6 and 12 hours (Figure $2.1 \mathrm{~F}$ and H). At 6 hours, 100 and 1000 pg/ml of TNF- $\alpha$ caused a decrease in cell viability of approximately 37\%. At 12 hours, 10, 100, and $1000 \mathrm{pg} / \mathrm{ml}$ caused a decrease in cell viability of approximately $50 \%$.

TNF- $\alpha$ caused a rapid decrease in mitochondrial function and a delayed decrease in cell viability in mouse primary cortical neurons

To confirm the rapid and profound effects of low doses of TNF- $\alpha$ on mitochondrial function was not specific to a transformed cell line, we exposed mouse primary cortical neurons to low doses 
of TNF- $\alpha$ for $1.5,3,6$, and 24 hours. After exposure to $100 \mathrm{pg} / \mathrm{ml}$ and $1000 \mathrm{pg} / \mathrm{ml}$ of TNF- $\alpha$ for 1.5 hours, basal respiration was decreased by $48 \%$ and $61 \%$, respectively without causing cell death (Figure 2.2 A and B). Acute exposure to low doses of TNF- $\alpha$ did not decrease cell viability until 24 hours after exposure to $1000 \mathrm{pg} / \mathrm{ml}$ of TNF- $\alpha$ (Figure $2.2 \mathrm{H}$ ). Since TNF- $\alpha$ rapidly decreases mitochondrial function prior to cell death in both HT-22 cells and mouse primary cortical neurons, we used the HT-22 cell line to determine the mechanism by which TNF- $\alpha$ rapidly affects mitochondrial function.

\section{HT-22 cells express TNF-R1 and TNF-R2}

It is known that TNF- $\alpha$ mediates its effects through TNF-R1 and TNF-R2. There is expression of TNF-R1 on all cell types, while expression of TNF-R2 is primarily on hemopoietic and endothelial cells (Maddahi et al., 2011). To determine the expression pattern of TNF- $\alpha$ receptors on HT-22 cells, flow cytometry analysis of HT-22 cells with antibodies specific for TNF-R1 and TNF-R2 was performed. HT-22 cells expressed both TNF-R1 (24.56\%) and TNF-R2 (87.17\%) under normal culture conditions (Supplemental Figure 2.2).

\section{TNF- $\alpha$ exerts its neurotoxic effects through TNF-RI}

Pre-treating HT-22 cells with 4, 6, or $8 \mathrm{ug} / \mathrm{ml}$ of TNF-R1 antibody for 1 hour ameliorated the TNF- $\alpha$ induced decrease in mitochondrial basal respiration (Figure 2.3 A), ATP production, and maximal respiration (data not shown) at 1.5 hours. As observed in Figure $2.1 \mathrm{~B}, \mathrm{TNF}-\alpha$ did not kill HT-22 cells at 1.5 hours (Figure $2.3 \mathrm{~B}$ and E). The decrease in cell viability caused by 100 $\mathrm{pg} / \mathrm{ml}$ of TNF- $\alpha$ at 3 hours was ameliorated when TNF-R1 was blocked (Figure 2.3 C). However, when HT-22 cells were pre-treated with 4, 6, or $8 \mathrm{ug} / \mathrm{ml}$ of TNF-R2 antibody, the 
neurotoxic effects of TNF- $\alpha$ on mitochondrial basal respiration at 1.5 hours (Figure 2.3 D) or cell viability at 3 hours (Figure $2.3 \mathrm{~F}$ ) was not affected.

TNF- $\alpha$ caused an increase in caspase 8 activity but not caspase 3/7 activity in HT-22 cells After exposure to $1,10,100,1000 \mathrm{pg} / \mathrm{ml}$ of TNF- $\alpha$ for 1.5 hours, caspase 8 and caspase $3 / 7$ activity was assessed with caspase 8 and caspase 3/7 luciferase assays. TNF- $\alpha$ caused a dosedependent increase in caspase 8 activity (Figure 2.4 A); however, caspase 3/7 activity was not affected by TNF- $\alpha$ exposure at this early time (Supplemental Figure 2.3). At the highest TNF- $\alpha$ concentration of $1000 \mathrm{pg} / \mathrm{ml}$, caspase 8 activity was increased by $46 \%$.

TNF- $\alpha$ caused a decrease in mitochondrial membrane potential in HT-22 cells Mitochondrial membrane potential dissipation results from the activation of caspases; thus, the increase in caspase 8 activity after 1.5 hours of exposure to TNF- $\alpha$ led us to hypothesize that TNF- $\alpha$ causes mitochondrial membrane potential to decrease as a result of the activation of caspase 8 . TNF- $\alpha$ exposure for 1.5 hours resulted in a significant dose-dependent decrease in mitochondrial membrane potential (Figure 2.4 B), and there was a significant correlation between the increase in caspase 8 activity and decrease in mitochondrial membrane potential (Figure 2.4 C).

$T N F-\alpha$ induced cytochrome c release from mitochondria in HT-22 cells

After exposure to 100 and $1000 \mathrm{pg} / \mathrm{ml}$ of TNF- $\alpha$ for $3,6,12$, and 24 hours, there was a dosedependent increase in cytochrome $c$ release from mitochondria (Figure 2.5). Exposure to TNF- $\alpha$ for 3, 12, and 24 hours with 100 and $1000 \mathrm{pg} / \mathrm{ml} \mathrm{TNF- \alpha}$ resulted in a significant increase in 
cytochrome c release, and after exposure for 6 hours only $1000 \mathrm{pg} / \mathrm{ml}$ of TNF- $\alpha$ resulted in a significant increase in cytochrome $c$ release $(\mathrm{p}<0.05)$.

\subsection{Discussion}

The present study shows, for the first time, that TNF- $\alpha$ rapidly and profoundly reduces neuronal cell mitochondrial function. It is widely accepted that mitochondria play a role in neuroinflammation and neurodegenerative CNS disorders such as multiple sclerosis, Alzheimer's disease, and Parkinson's disease (Di Filippo et al., 2010). With an insult like ischemic stroke, activation of microglia occurs that leads to the release of TNF- $\alpha$ along with other proinflammatory cytokines (Davalos et al., 2005). Pro-inflammatory cytokines can activate mitochondria-induced apoptosis leading to cell death (Huang et al., 2005). Our findings suggest that TNF- $\alpha$ exposure caused neuronal mitochondrial dysfunction as expressed by decreased mitochondrial respiration as early as 1.5 hours of exposure.

Neurons have a high ATP demand (Zhu et al., 2012), and a temporary reduction in ATP production results in a profound decrease in neuronal viability (Simpkins et al., 2010). Fiskum et al. (1999) hypothesized that neuronal death that occurs at the core of an infarct after ischemia results from the decrease in mitochondrial and glycolytic ATP production. TNF- $\alpha$ is known to exacerbate infarct size in pre-clinical models of stroke, and this may be due to the profound and rapid decrease in ATP production, caused by TNF- $\alpha$ release in the evolving ischemic core. Although TNF-R2 is primarily expressed on immune cells, and the expression of TNF-R1 is constitutive on all cell types, HT-22 cells express both receptors (Maddahi et al., 2011). TNFR2 plays a major role in the lymphoid system and requires membrane bound TNF- $\alpha$ for full activation; thus, we hypothesized that TNF- $\alpha$ affects mitochondrial function through TNF-R1 
because soluble TNF- $\alpha$ signaling is primarily through TNF-R1 (Wajant et al., 2003; Maddahi et al., 2011). Furthermore, there is no correlation between the number of receptors present on a cell and the magnitude of the TNF-induced response (Beyaert and Fiers, 1994). Blocking TNFR1 ameliorated the neurotoxic effects of TNF- $\alpha$ on mitochondrial respiration and cell viability, confirming TNF- $\alpha$ exerts neurotoxic effects through TNF-R1 not TNF-R2. To further understand the neurotoxic mechanisms of TNF- $\alpha$, we assessed caspase 8 and caspase 3/7 activity because TNF- $\alpha$ can signal through TNF-R1 and activate caspase 8 or caspase 3/7 (Baud and Karin, 2001; McCoy and Tansey, 2008). The activation of caspase 8 causes mitochondrialinduced apoptosis through the cleavage of Bcl-2-associated X protein (BAX) and BH3 interacting-domain death agonist (BID) (Aggarwal, 2003; McCoy and Tansey, 2008). Caspase 8 activity was increased in a dose-dependent manner after exposure to TNF- $\alpha$ for 1.5 hours; however, caspase $3 / 7$ activity was not affected at this early time. The increase in caspase 8 activity led us to hypothesize that mitochondrial membrane potential should decrease if caspase 8 is initiating mitochondrial-induced apoptosis (Green and Kroemer, 2004). The TMRE mitochondrial membrane potential assay showed a significant dose-dependent decrease in mitochondrial membrane potential after 1.5 hour of TNF- $\alpha$ exposure. Furthermore, with a collapse of mitochondrial membrane potential there should be an increase in cytochrome $c$ release from the mitochondria, due to the cleavage of BAX and BID (Baud and Karin, 2001; Aggarwal, 2003; McCoy and Tansey, 2008). After exposure to $100 \mathrm{pg} / \mathrm{ml}$ of TNF- $\alpha$ for 3 and 24 hours, there was an $11 \%$ and $68 \%$ increase in cytochrome $c$ release, respectively, from mitochondria. Our observation of a $19.7 \%$ decrease in mitochondrial membrane potential and the $30 \%$ decrease in ATP production at 1.5 hours of treatment with $100 \mathrm{pg} / \mathrm{ml} \mathrm{TNF-} \alpha$ indicates that sufficient cytochrome $c$ is released to induce apoptosis (Sas et al., 2007). Once cytochrome $c$ is released, cytochrome $c$ interacts with apoptotic protease-activating factor-1 (Apaf-1), procaspase-9, and 
dATP forming an apoptosome (Sas et al., 2007). The formation of the apoptosome allows for the activation of caspase-9, which activates procaspase-3, and results in the induction of apoptosis (Sas et al., 2007).

HT-22 cells and primary mouse cortical neurons both showed a rapid mitochondrial impairment and subsequent cell death in response to low concentrations of TNF- $\alpha$. However, there was a notable difference between the two cell types in the timing of the TNF- $\alpha$ induced cell death. Whereas HT-22 cells died within 3 hours of exposure, primary neurons showed cell loss only at 24 hours. The reason for this delayed response of primary neurons is not clear, but is consistent with the comparative resistance of primary neurons to TNF- $\alpha$ in other studies (Reimann-Philipp et al., 2001; Zhao et al., 2001). We suspect, but do not have evidence to support, that primary neurons may have inherent defense mechanisms that resist the neurotoxic effects of a single cytokine, like TNF- $\alpha$ (Tamatani et al., 1999).

Numerous studies reported an association between increased levels of TNF- $\alpha$ mRNA and protein and an exacerbation of ischemic injury (Di Filippo et al., 2010). Administration of TNF- $\alpha$ blocking antibodies or soluble TNF-R1 prior to inducing stroke reduces infarct volume (Murakami et al., 2005; Di Filippo et al., 2010). Our findings may provide the mechanism of TNF- $\alpha$ exacerbation of stroke damage and shows that TNF- $\alpha$ elicits neurotoxic effects by inducing a rapid mitochondrial dysfunction mediated through TNF-R1 signaling subsequently activating caspase 8 and leading to the release of cytochrome $c$.

Current clinical treatment of ischemic stroke concentrates on dissolving and/or removing the clot, without much focus on the secondary damage that occurs as part of the inflammatory ischemic cascade (Works et al., 2013). Inflammation is known to play a major role in the 
pathophysiology of stroke and progression of damage post-stroke (Vila et al., 2000a; Iadecola and Anrather, 2011). The identification of a new therapeutic to reduce brain damage due to inflammation post-stroke would be clinically useful. However, TNF- $\alpha$ has both pro-apoptotic and anti-apoptotic activities, and both roles are important physiologically (Aggarwal, 2003). When the expression of TNF- $\alpha$ is profound and prolonged, such as after stroke, it has harmful effects (Aggarwal, 2003). Thus, understanding the neurotoxic mechanism of TNF- $\alpha$ is important to further advance its potential as a therapeutic target.

As with all in vitro studies, our studies have some limitations, and we interpreted our results with caution. First, TNF- $\alpha$ was administered at discrete doses (1-1000 pg/ml) for 1.5 to 24 hours. Post- stroke concentrations change over time and are elevated in patients within 6 hours of symptom onset and stay elevated for up to 10 days (Zaremba and Losy, 2001; Intiso et al., 2003). Furthermore, TNF- $\alpha$ mRNA is increased in the ischemic core within 1 hour, and TNF- $\alpha$ immunoreactive protein is seen within 2-6 hours in the brain after middle cerebral artery occlusion, (MCAO) in preclinical models (Liu et al., 1994; Botchkina et al., 1997). We observed mitochondrial impairment with clinically relevant concentrations of TNF- $\alpha$ over a critical time window post-stroke. An additional limitation is the use of cell culture models such as a transformed cell line and a primary mouse neuronal culture. Both cell types are models for neurons, but do not have astrocytes, microglia, or endothelial cells; thus, the results should be taken with caution. In view of the similarity between the two models, we believe we have modeled the effects of TNF- $\alpha$ on nerve cells. Finally, we did not evaluate cleavage of BAX and BID. We assumed the cleavage occurred because caspase 8 cleaves BAX and BID, resulting in cytochrome $c$ release. Our results support that exposure to TNF- $\alpha$ at 1.5 hours results in an 
increase in caspase 8 activity, and by 3 hours we observed a significant release of cytochrome $c$ from the mitochondria into the cytosol.

This study shows, for the first time, a rapid and profound mitochondrial dysfunction in neurons with acute exposure to low doses of TNF- $\alpha$. This neurotoxic effect of TNF- $\alpha$ appears to be mediated by TNF-R1, leading to caspase activation, mitochondrial membrane potential collapse, and mitochondrial release of cytochrome $c$. Collectively these data help to elucidate the neurotoxic mechanisms of TNF- $\alpha$, and thereby, provides potential targets for cytokine directed therapy for neuroprotection. 


\section{Figure Legends}

Figure 2.1: Effects of TNF- $\alpha$ on basal respiration and cell viability in HT-22 cells. (A) Basal respiration after exposure to TNF- $\alpha$ for 1.5 hours. (B) Cell viability after exposure to TNF- $\alpha$ for 1.5 hours. (C) Basal respiration after exposure to TNF- $\alpha$ for 3 hours. (D) Cell viability after exposure to TNF- $\alpha$ for 3 hours. (E) Basal respiration after exposure to TNF- $\alpha$ for 6 hours. (F) Cell viability after exposure to TNF- $\alpha$ for 6 hours. (G) Basal respiration after exposure to TNF$\alpha$ for 12 hours. (H) Cell viability after exposure to TNF- $\alpha$ for 12 hours. Analysis of variance and Dunnett post-hoc tests were used to assess significance. $* \mathrm{p}<0.05 ; * * \mathrm{p}<0.01 ; * * * \mathrm{p}<0.001$. Depicted are mean + SEM for $n=6 /$ group

Figure 2.2: Effects of TNF- $\alpha$ on basal respiration and cell viability in mouse primary cortical neurons. (A) Basal respiration after exposure to TNF- $\alpha$ for 1.5 hours. (B) Cell viability after exposure to TNF- $\alpha$ for 1.5 hours. (C) Basal respiration after exposure to TNF- $\alpha$ for 3 hours. (D) Cell viability after exposure to TNF- $\alpha$ for 3 hours. (E) Basal respiration after exposure to TNF- $\alpha$ for 6 hours. (F) Cell viability after exposure to TNF- $\alpha$ for 6 hours. (G) Basal respiration after exposure to TNF- $\alpha$ for 24 hours. (H) Cell viability after exposure to TNF- $\alpha$ for 24 hours. Analysis of variance and Dunnett post-hoc tests were used to assess significance. ${ }^{*} \mathrm{p}<0.05$; ** $\mathrm{p}<0.01$ *** $^{*} \mathrm{p}<0.001$. Depicted are mean + SEM for $\mathrm{n}=6$ /group. When SEM is not depicted, it is too small for representation.

Figure 2.3: Effects of blocking TNF-R1 or TNF-R2 on TNF- $\alpha$ induced changes in mitochondrial basal respiration and cell viability. (A) Effects of TNF-R1 antibody on TNF- $\alpha$ induced changes in mitochondrial basal respiration at 1.5 hours. (B) Effects of TNF-R1 antibody on TNF- $\alpha$ induced changes in cell viability at 1.5 hours. (C) Effects of TNF-R1 
antibody on TNF- $\alpha$ induced changes in cell viability at 3 hours. (D) Effects of TNF-R2 antibody on TNF- $\alpha$ induced changes in mitochondrial basal respiration at 1.5 hours. (E) Effects of TNFR2 antibody on TNF- $\alpha$ induced changes in cell viability at 1.5 hours. (F) Effects of TNF-R2 antibody on TNF- $\alpha$ induced changes in cell viability at 3 hours. Analysis of variance and Dunnett post-hoc tests were used to assess significance, ${ }^{*} \mathrm{p}<0.05$, *** $\mathrm{p}<0.001 ; \# \# \mathrm{p}<0.01$; \#\#\# p< 0.001; * compared to Vehicle; \# compared to $100 \mathrm{pg} / \mathrm{ml}$ TNF- $\alpha$; Depicted are mean + SEM for $n=6 /$ group.

Figure 2.4: (A) Effects of 1.5 hours of exposure to TNF- $\alpha$ on caspase 8 activity. Analysis of variance and Dunnett post-hoc tests were used to assess significance, $* * * p<0.001$ (B) Effects of 1.5 hours of treatment with TNF- $\alpha$ on mitochondrial membrane potential. Analysis of variance with post-test for linear trend found a significant dose-dependent decrease in mitochondrial membrane potential, $\mathrm{R}$ square $=0.3571, \mathrm{p}=0.0163$ Pearson's correlation between caspase 8 activity and mitochondrial membrane potential. $\mathrm{R}$ square $=0.9437, \mathrm{p}=$ 0.086. Depicted are mean + SEM for $n=6 /$ group.

Figure 2.5: Effects of TNF- $\alpha$ on cytochrome $c$ release. (A) Exposure to TNF- $\alpha$ for 3 hours. (B) Exposure to TNF- $\alpha$ for 6 hours. (C) Exposure to TNF- $\alpha$ for 12 hours. (D) Exposure to TNF- $\alpha$ for 24 hours. Analysis of variance and Dunnett post-hoc tests were used to assess significance. * $\mathrm{p}<0.05 ; * * \mathrm{p}<0.01$; *** $\mathrm{p}<0.001$. Depicted are mean + SEM for $\mathrm{n}=6$ /group. When SEM is not depicted, it is too small for representation. 
A Basal Respiration

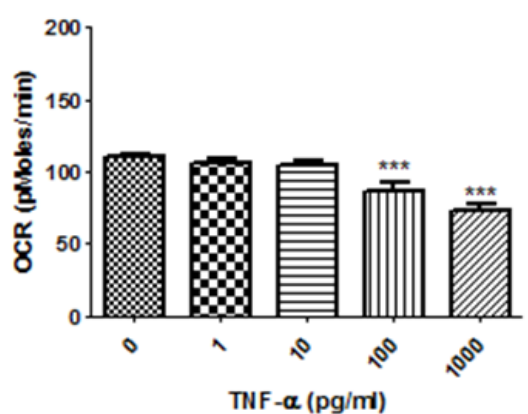

C

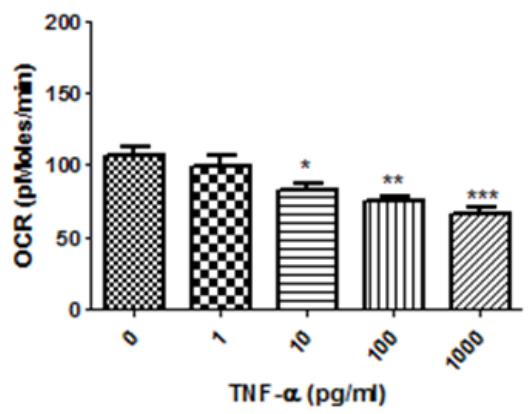

E

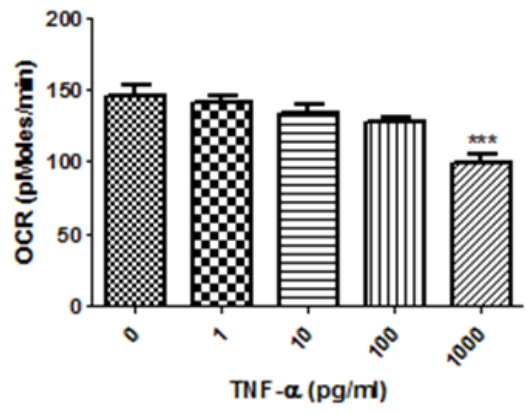

G

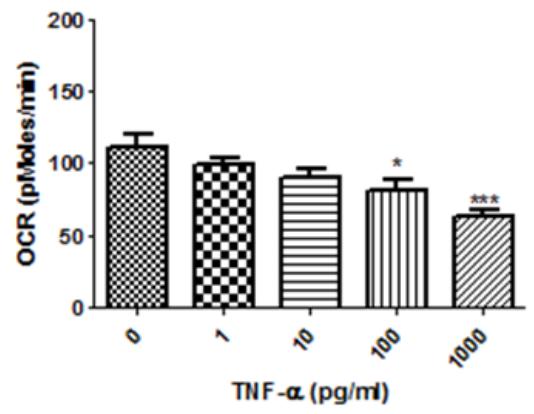

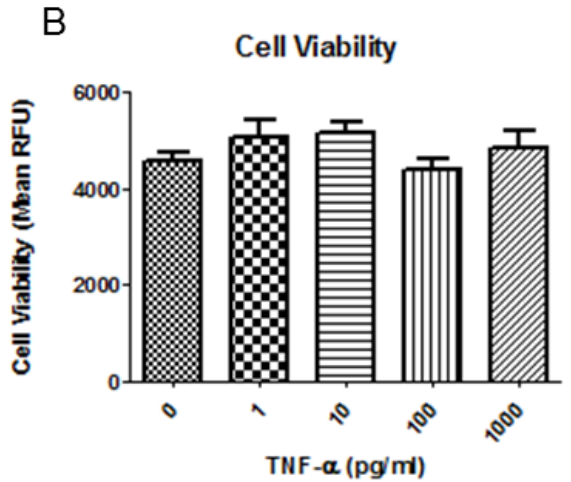

D

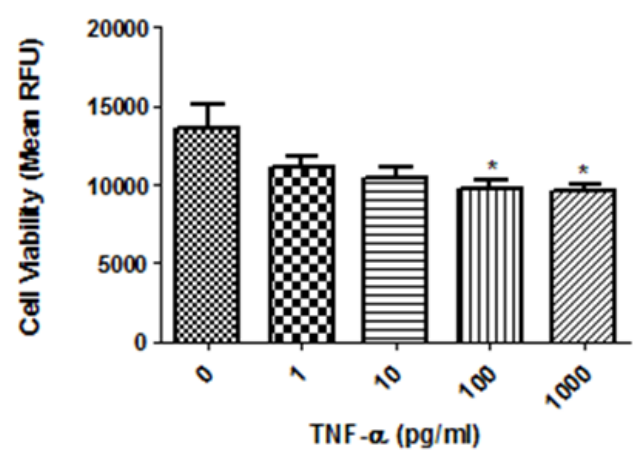

F

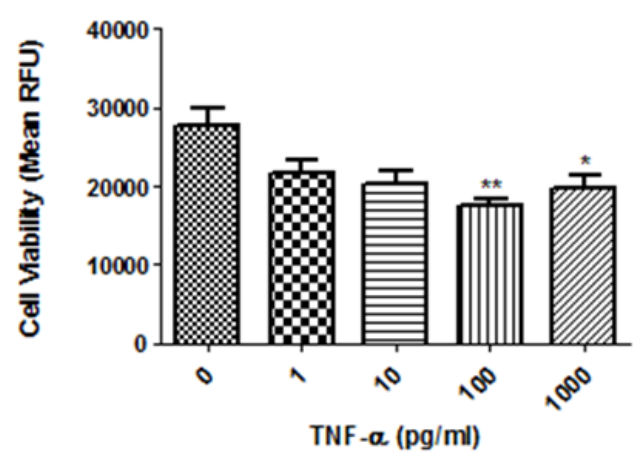

$\mathrm{H}$

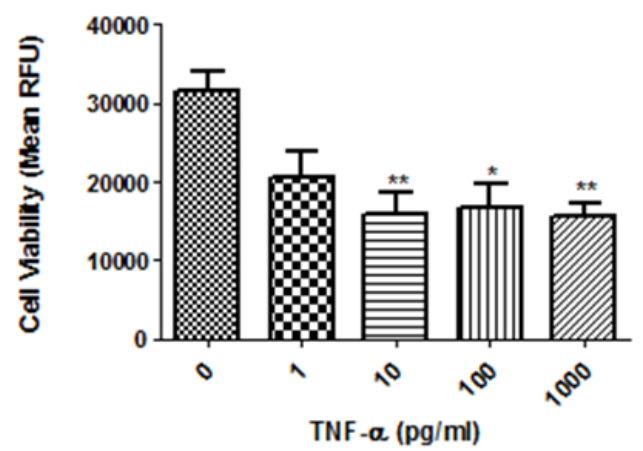

Figure 2.1 

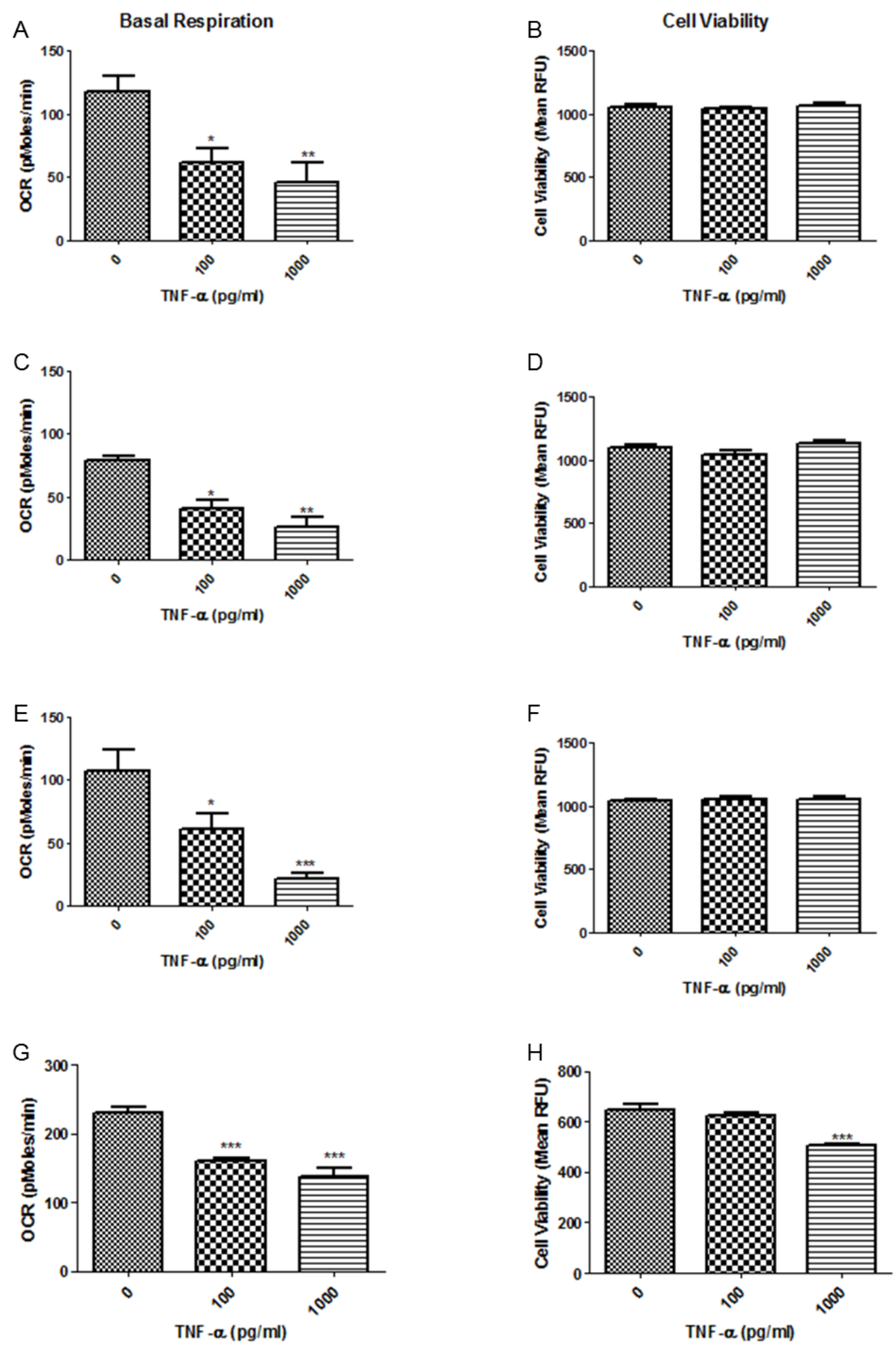

Figure 2.2 

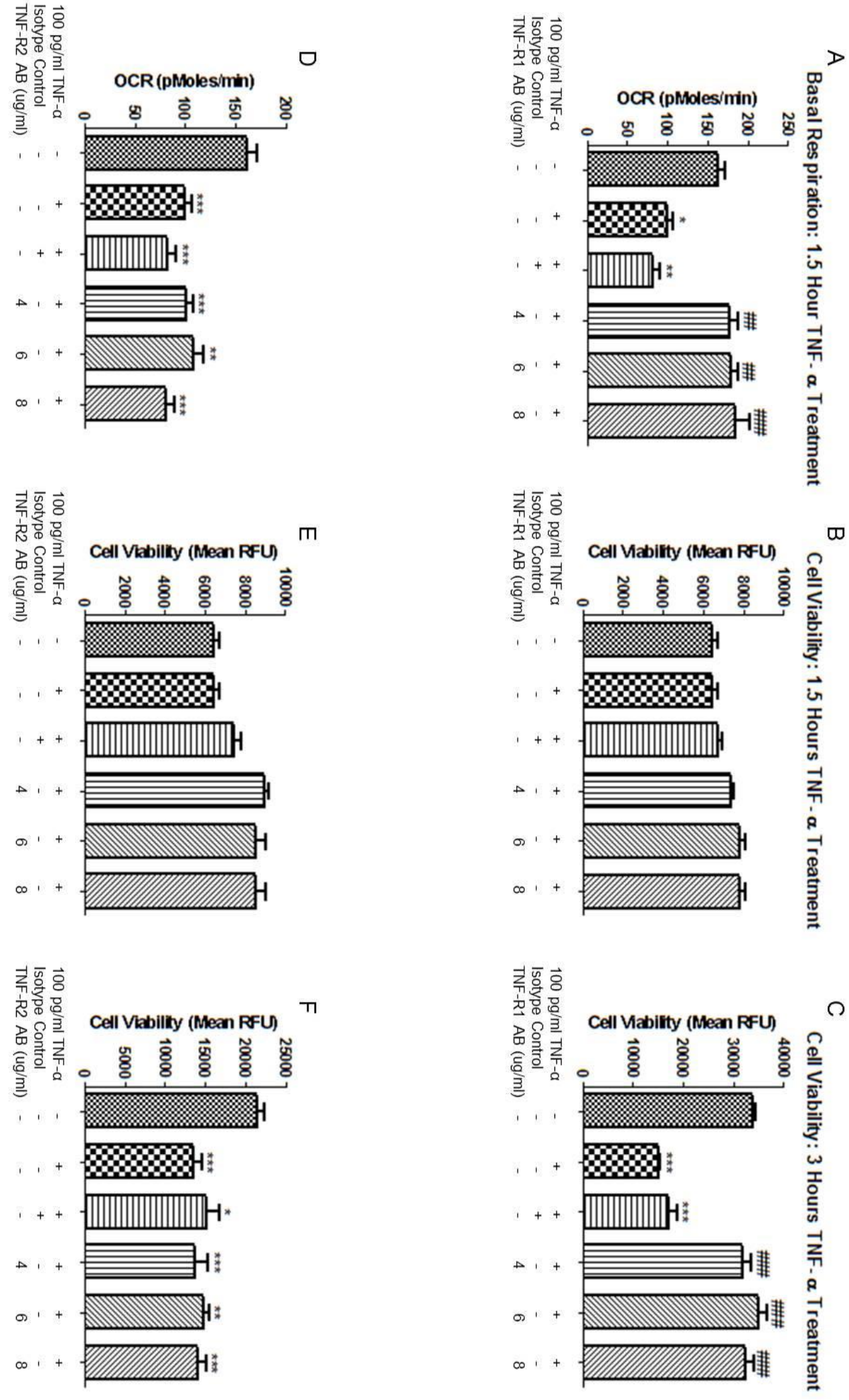

Figure 2.3 

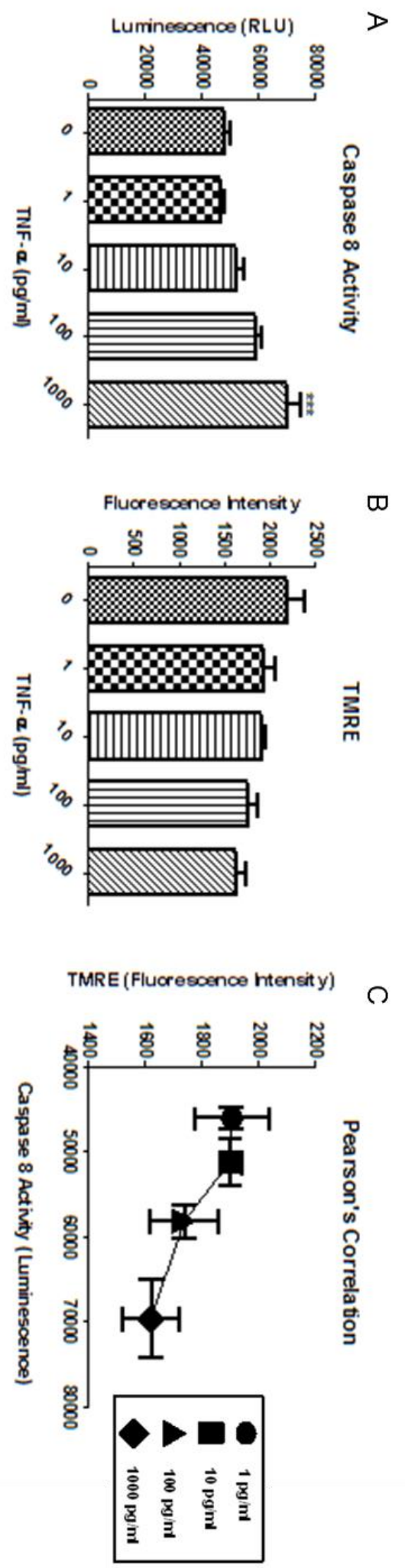

Figure 2.4 

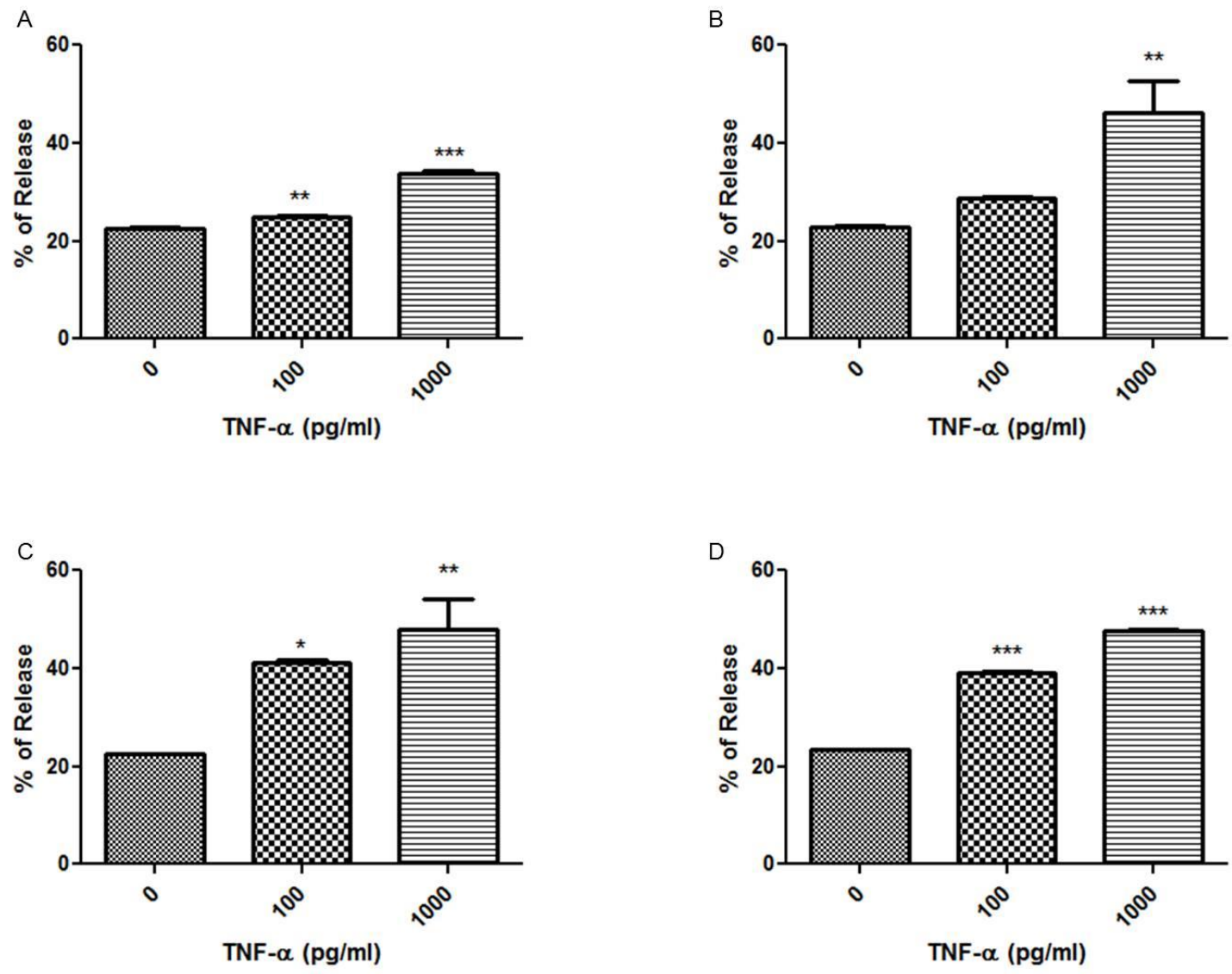

Figure 2.5 


\section{Supplemental Figure Legends}

Supplemental Figure 2.1: Effects of TNF- $\alpha$ on ATP production and maximal respiration. (A) Exposure to TNF- $\alpha$ for 1.5 hours. (B) Exposure to TNF- $\alpha$ for 3 hours. (C) Exposure to TNF- $\alpha$ for 6 hours. (D) Exposure to TNF- $\alpha$ for 12 hours. Analysis of variance and Dunnett post-hoc tests were used to assess significance. ${ }^{*} \mathrm{p}<0.05 ; * * \mathrm{p}<0.01 ; * * * \mathrm{p}<0.001$. Depicted are mean + SEM for $n=6 /$ group.

Supplemental Figure 2.2: Flow cytometry analysis of HT-22 cell expression of TNF-R1 and TNF-R2.

Supplemental Figure 2.3: Effects of 1.5 hours of exposure to TNF- $\alpha$ on caspase 3/7 activity. Analysis of variance did not show a significant difference among the groups. Depicted are mean + SEM for $n=6$ /group. When SEM is not depicted, it is too small for representation. 
A ATP Production

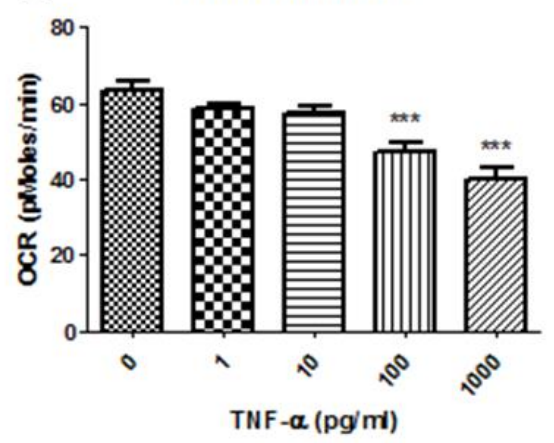

B

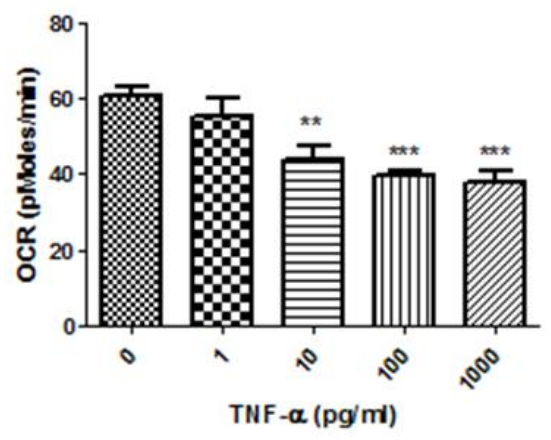

C

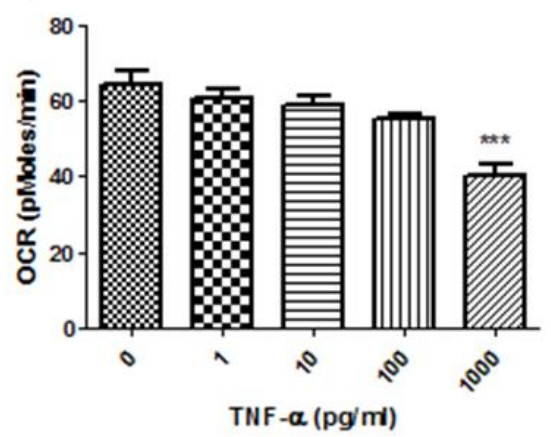

D

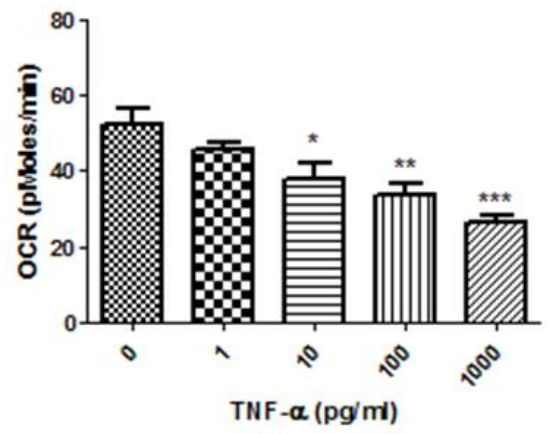

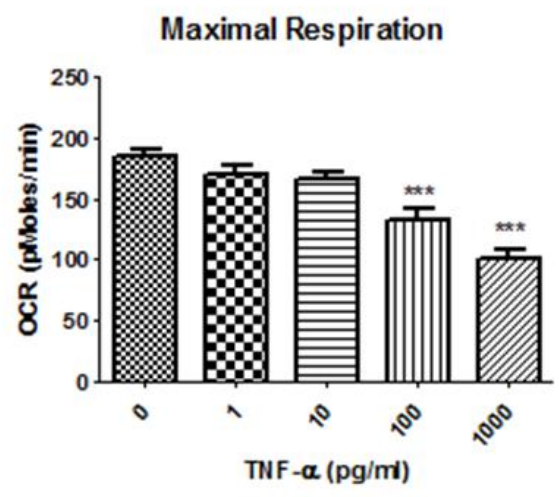
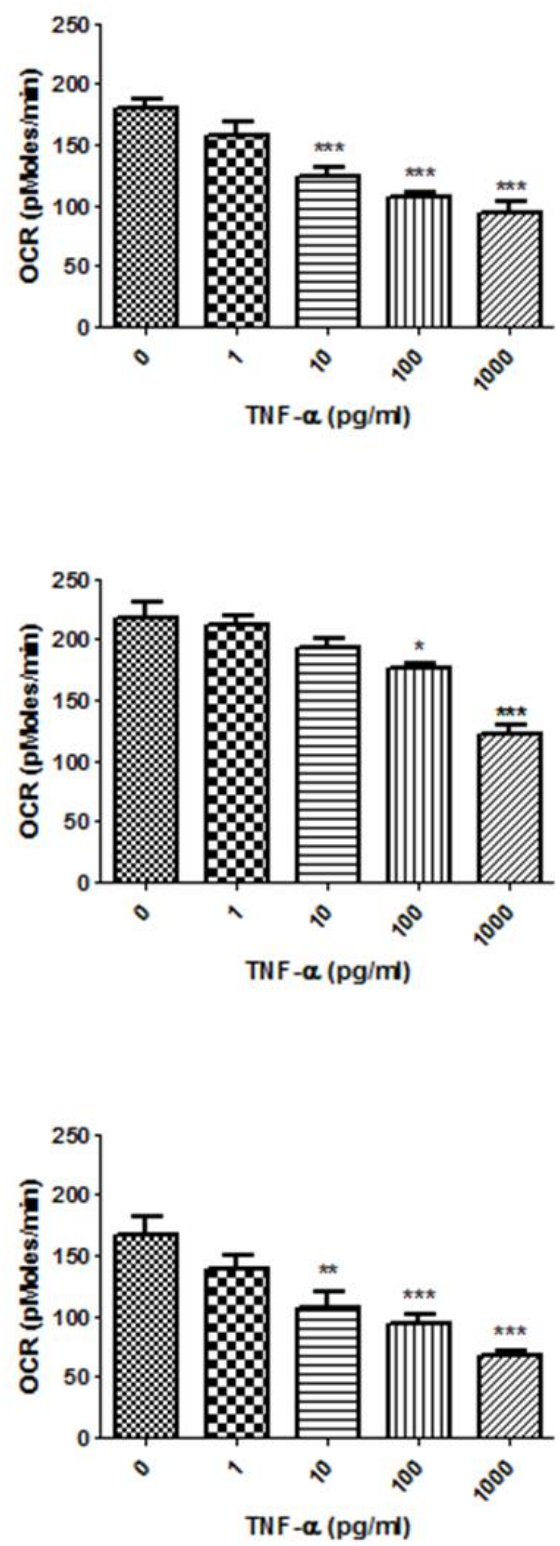

Supplemental Figure 2.1 


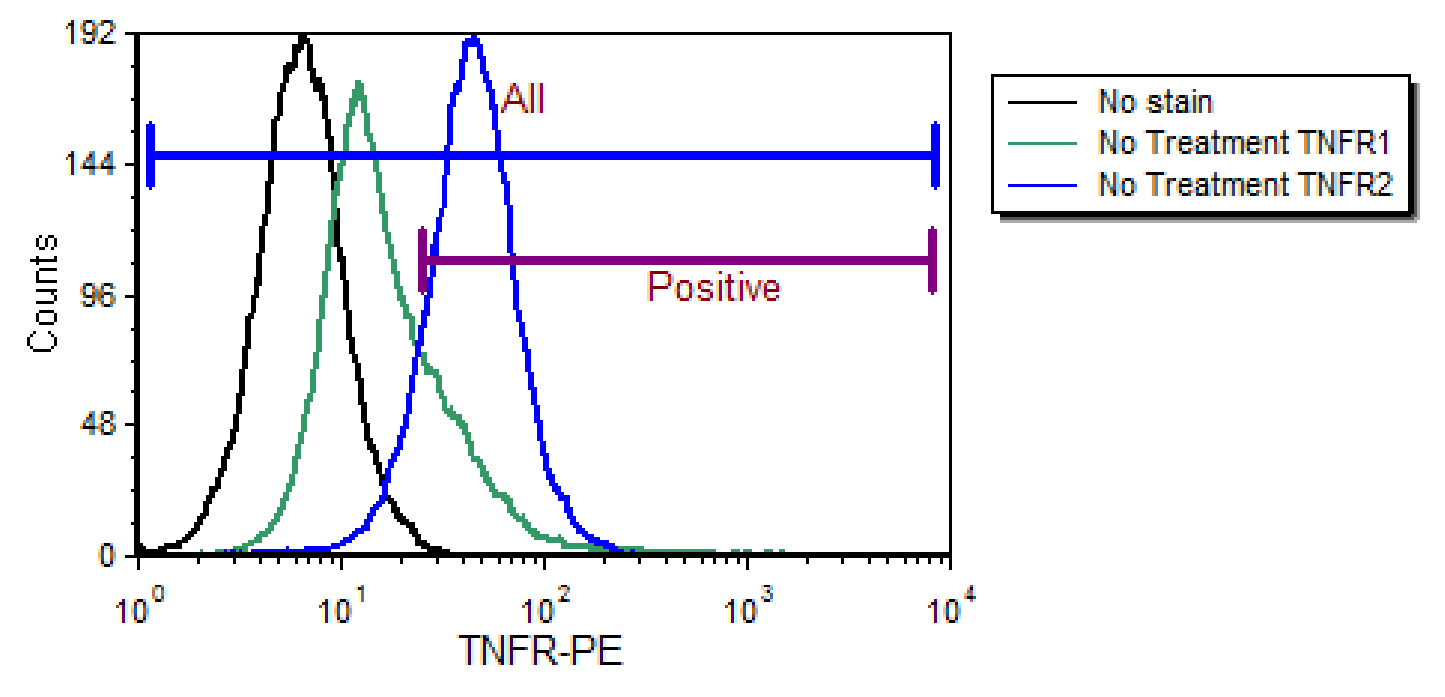

Supplemental Figure 2.2 


\section{Caspase 3 and 7 Activity}

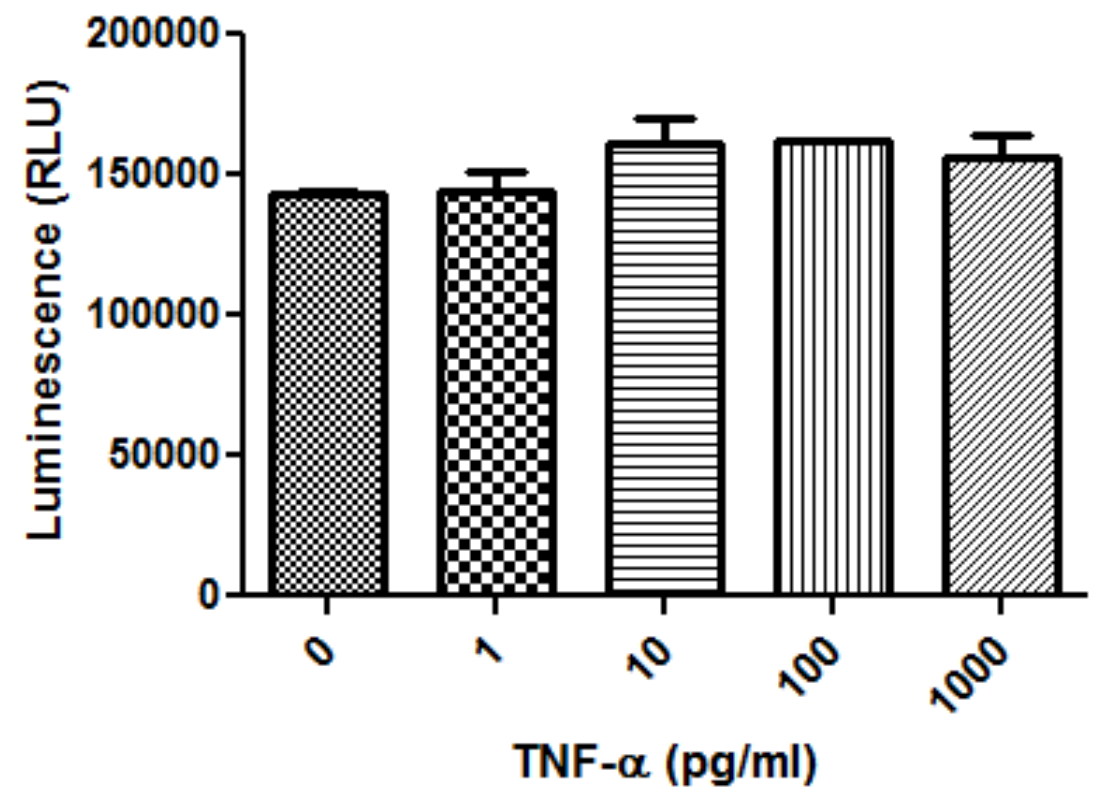

Supplemental Figure 2.3 


\section{Chapter 3}

\section{Mitochondrial Crisis in Cerebrovascular Endothelial Cells Opens the Blood-Brain Barrier}

Danielle N. Doll, Heng Hu, MD, Jiahong Sun, Sara E. Lewis, MS, James W. Simpkins, PhD, Xuefang Ren

Doll DN, Hu H, Sun J, Lewis SE, Simpkins JW, Ren X. (2015) Mitochondrial Crisis in Cerebrovascular Endothelial Cells Opens the Blood-Brain Barrier. Stroke, 46, 1681 - 1689. 


\subsection{Abstract}

Background and Purpose--The blood-brain barrier (BBB) is a selectively permeable cerebrovascular endothelial barrier that maintains homeostasis between the periphery and central nervous system (CNS). BBB disruption is a consequence of ischemic stroke and BBB permeability can be altered by infection/inflammation, but the complex cellular and molecular changes that result in this $\mathrm{BBB}$ alteration need to be elucidated to determine mechanisms.

Methods - Infection mimic (LPS) challenge on infarct volume, BBB permeability, infiltrated neutrophils and functional outcomes following murine transient middle cerebral artery occlusion (tMCAO) in vivo; mitochondrial evaluation of cerebrovascular endothelial cells (CVECs) challenged by LPS in vitro; pharmacological inhibition of mitochondria on BBB permeability in vitro and in vivo; the effects of mitochondrial inhibitor on BBB permeability, infarct volume and functional outcomes following tMCAO.

Results--We report here that LPS worsens ischemic stroke outcome and increases BBB permeability following tMCAO in mice. Further, we elucidate a novel mechanism that compromised mitochondrial function accounts for increased BBB permeability as evidenced by: LPS-induced reductions in oxidative phosphorylation and subunit expression of respiratory chain complexes in CVECs, a compromised BBB permeability induced by pharmacological inhibition of mitochondrial function in CVECs in vitro and in an in vivo animal model, and worsened stroke outcomes in tMCAO mice following inhibition of mitochondrial function.

Conclusions--We concluded that mitochondria are key players in BBB permeability. These novel findings suggest a potential new therapeutic strategy for ischemic stroke by endothelial cell mitochondrial regulation. 


\subsection{Introduction}

The blood-brain barrier (BBB) is a highly specialized vascular interface that maintains homeostasis in brain by separating the blood compartment from the central nervous system (CNS). Disruption of cerebrovascular endothelial cells not only allows entry of unwanted solutes into brain, but also disrupts the normal CNS entry route of critical nutrients (Rubin and Staddon, 1999; Gonzalez-Mariscal et al., 2000). Disruption and/or dysfunction of the BBB has been observed in cerebrovascular diseases and neurodegenerative disorders such as stroke, Alzheimer's disease (AD), Parkinson disease (PD), Huntington's disease and epilepsy (Tajes et al., 2014).

Stroke is the second leading cause of death and the leading cause of disability worldwide (Feigin et al., 2014). It is estimated that $30-40 \%$ of all strokes occur during or recently after an acute infection (Grau et al., 1995b). Acute infections initiate rapid inflammation, and post-stroke infections worsen outcomes in patients (Grau et al., 1995a) and in animal models (McColl et al., 2007). However, the mechanisms of this exacerbation by infection on acute stroke outcome are not completely understood.

Ischemia and reperfusion events result in complex cellular and molecular changes that further need to be elucidated. Thus far, studies have provided evidence that the release of oxidants, proteolytic enzymes and inflammatory cytokines alter BBB permeability. The BBB excludes the majority of bacteria; however, similar to ischemia and reperfusion, inflammation induced by bacteria alters BBB permeability. Lipopolysaccharide (LPS) has been shown to disrupt the BBB in vivo and in vitro, however, how LPS exerts its effects on the BBB is under debate (Banks and 
Erickson, 2010). Previous studies found that an LPS challenge results in a larger infarct volume (McColl et al., 2007), impairs survival and disrupts BBB (Denes et al., 2011) in experimental stroke models, through mechanisms that are proposed to involve cytokines.

In the present study, we provide the first evidence that the exacerbation of stroke outcome by a bacterial infection mimic is due to a novel mechanism of compromised cerebrovascular endothelial mitochondrial function. Potential deleterious mitochondrial responses to ischemia have been observed, such as rapid changes in ATP, the release of cytochrome $c$, and the induction of the mitochondrial permeability transition (Sims and Anderson, 2002; Sims and Muyderman, 2010). It is apparent mitochondria play a pivotal role during ischemia; however, the contributions of mitochondrial dysfunction in non-neuronal cells and the interactions between these cells and neurons are poorly understood. Additionally, the involvement of mitochondria in ischemic damage has not been fully elucidated. We show that pharmacological disruption of endothelial mitochondrial function recapitulates all aspects of the LPS exacerbation of stroke, including disruption of the BBB, and worsening of stroke outcome. This discovery could provide a previously unknown mitochondrial-dependent mechanism for acute stroke damage that may offer new therapeutic directions for the treatment of acute stroke.

\subsection{Methods}

Mice

All procedures were conducted according to the criteria approved by the Institutional Animal Care and Use Committees (IACUC) at the West Virginia University (WVU). C57/BL6J male mice (3-4 months old, 25-30g, Jackson Laboratories) were used for all studies. 


\section{Anesthesia}

All surgical anesthesia was induced with 4-5\% isoflurane until the animal showed no response to a toe pinch, and was maintained with 1-2\% isoflurane via face-mask in O2-enriched air.

\section{Randomization and blinding of the animal experiments}

To assign pre-treatments of mice, we numbered the animals and applied a simple randomization by using excel-generated random numbers. To avoid biases, we also assured that different pretreatments were performed on the same day. The experimenters were blinded to the pretreatments and data analysis.

\section{Drug administration}

In a subgroup of mice, LPS (Escherichia coli 055:B5, $100 \mu \mathrm{g} / \mathrm{kg}$, Sigma) dissolved in saline (B. Braun Medical Inc. Irvine, California) was injected intraperitoneally $30 \mathrm{~min}$ prior to tMCAO or sham surgery. In another subgroup of mice, rotenone (1 $\mathrm{mg} / \mathrm{kg}$, Sigma) was administered intraperitoneally $30 \mathrm{~min}$ prior to tMCAO. In the EA model, rotenone $(2 \mathrm{mg} / \mathrm{ml}, 2 \mu \mathrm{l})$ was topically applied to the epidural membrane. An equal volume of saline was administered to control mice.

\section{Ischemic model and sham surgery}

We performed focal cerebral ischemia for $30 \mathrm{~min}$ or $60 \mathrm{~min}$ by occlusion of the right middle cerebral artery with a 6.0 monofilament suture (Doccol, Sharon, Massachusetts). We used laser Doppler flowmetry (Moor instruments, United Kingdom) to detect regional cerebral blood flow 
and confirm a successful occlusion ( $>70 \%$ decrease in flow). Rectal body temperature was maintained at $37 \pm 0.5{ }^{\circ} \mathrm{C}$ during surgery. Mice were euthanized at several time points as indicated in the text.

\section{Neurological deficits}

Neurologic deficit was determined daily before and after tMCAO according to a 0-5 point scale neurological score system as published (Harhausen et al., 2010).

\section{Epidural application (EA) model}

We induced anesthesia with $4 \%$ isofluorane and maintained with $1 \%$ isofluorane. We placed the animal in a prone position under a stereo dissecting microscope and made an incision down the midline of the head and retracted the skin then removed the fascia from the skull. Using a sharp blade, we thinned an area of the skull approximately $0.3-0.5 \mathrm{~mm}$ in diameter, divided the thinned area into several segments, performed a craniotomy by gently removing the thinned skull segments, applied drug or vehicle, and closed the incision.

\section{Exclusion criteria for animal experiments}

The exclusion criteria for tMCAO were: 1) regional cerebral blood flow decreases $<70 \%$ during occlusion as detected by laser Doppler flowmetry; 2) surgery time lasts more than 30 minutes; 3) no neurological deficits 3 hour after MCAO (neurological score 0); 4) no infarction in the MCA territory on TTC staining; 5) subarachnoid hemorrhage on postmortem examination; and 6) substantial ambient temperature change in the animal facility. Animals which died prior to the 
planned time of assessments were postmortem examined for subarachnoid hemorrhage, and the mortality was recorded.

The exclusion criteria for EA model were: 1) damaged epidural membrane, 2) bleeding from the epidural membrane; 3) the leakage of cerebrospinal fluid from the epidural membrane; 4) body temperature $>39.5^{\circ} \mathrm{C}$ within post-surgery 3 hours; and 5) death.

In this study, 7 mice were excluded: 2 mice (one vehicle and one LPS pre-treatment) because of subarachnoid hemorrhage, 4 mice ( 2 vehicle and 2 LPS pre-treatment) because the animal facility air conditioner broke down on the day that the experiments were performed, 1 mouse (pre-treated with rotenone) because LDF did not reach $70 \%$ reduction during the occlusion. The animal numbers included in the results are 18 vehicle and 23 LPS for 30 min tMCAO mice (4 LPS pretreated mice died), 12 vehicle and 12 rotenone for 60 min tMCAO mice, 5 vehicle and 5 rotenone for EA mice. No animals were excluded in EA model.

\section{Analysis of brain infarct volume}

Mice were euthanized with isofluorane. We removed the brains and cut $2 \mathrm{~mm}$ coronal sections with a mouse brain matrix. We stained the sections with 2\% 2,3,5-triphenyltetrazolium chloride (TTC, Sigma, Saint Louis, Missouri) in phosphate buffer solution (PBS) at $37^{\circ} \mathrm{C}$ for 30 minutes then fixed the tissue in $10 \%$ formalin phosphate buffer for digital photograph. We analyzed the digitized image of each brain section using a computerized image analysis software (ImageJ, $\mathrm{NIH}$ ) in a double blinded manner. To minimize the effect of brain edema, the volume was expressed as a percentage of contralateral cortex, striatum, and total hemisphere. 
Blood-brain barrier $(B B B)$ permeability assay in vivo

The permeability of the BBB was determined by measuring the penetration of Evans blue (Sigma) in the brain tissue. Evans blue ( $2 \%$ in saline, $4 \mathrm{ml} / \mathrm{kg}$ body weight) was administered intravenously via the tail vein one hour prior to measurement. The anesthetized animals were perfused transcardially with saline before sampling. Each sample was weighed and homogenized with $400 \mu \mathrm{l}$ PBS, then precipitated by $50 \%$ trichloroacetic acid overnight. The samples were centrifuged for 30 minutes at $10,000 \mathrm{rpm}$ to pellet brain tissue. Absorption of the supernatant was measured at a wavelength of $610 \mathrm{~nm}$ with a plate reader (BioTek, Winooski, Vermont). The extravasation of Evans blue was quantified as $\mu \mathrm{g} / \mathrm{g}$ brain tissue with an Evans blue standardized curve.

\section{Isolation of immune cells in the blood, spleen and brain}

Blood was collected via cardiac puncture then brain and spleen were sampled after transcardial perfusion with saline. Peripheral blood mononuclear cells and splenocytes were prepared by using red cell lysis buffer (eBioscience, San Diego, California). For brain immune cells, the forebrain was dissected from the cerebellum and suspended in RPMI-1640 medium (Corning, Pittsburgh, Pennsylvania). The suspension was digested with type IV collagenase $(1 \mathrm{mg} / \mathrm{ml}, \mathrm{MP}$ Biomedicals, Solon, Ohio) and DNase I (50 $\mu \mathrm{g} / \mathrm{ml}$, Roche Diagnostics, Indianapolis, Indiana) at $37^{\circ} \mathrm{C}$ for $45 \mathrm{~min}$, then immune cells were isolated by $37-70 \%$ Percoll (GE Healthcare, Piscataway, New Jersey) density gradient centrifugation and collected from the interface. Single cell suspension was washed with staining buffer (PBS containing $0.1 \% \mathrm{NaN} 3$ and $2 \%$ FCS) and stained with CD45 (30-F11, eBioscience), CD11b (M1/70, eBioscience), and Gr1 (IA8, BD Bioscience). Propidium iodide (PI, $2 \mu \mathrm{g} / \mathrm{ml}$, Sigma) was used to exclude dead cells. Appropriate 
isotype control antibodies were applied to set quadrants for calculating the percentage of positive cells. Data were acquired on FACS Calibur (BD) and analyzed with FlowJo software (TreeStar, Ashland, Oregon).

\section{Cell culture}

The bEnd.3 cell line (CRL-2299 from ATCC, Manassas, Virginia) was originally derived from mouse brain cortex endothelial cells and confirmed by the observed major phenotypic features of the blood-brain barrier.(Brown et al., 2007a) Passages 25-30 were used in the study. The bEnd.3 cells were routinely grown in high glucose Dulbecco's modified Eagle's medium (DMEM, ATCC) supplemented with 10\% FCS and 1\% penicillin/streptomycin (Hyclone, South Logan, Utah) at $37{ }^{\circ} \mathrm{C}$ in $5 \% \mathrm{CO} 2$ humid atmosphere. Mouse primary brain microvascular endothelial cells (B129-7023 from CellBiologics, Chicago, Illinois) were routinely grown in complete Endothelial Cell Medium (Cell Biologics, M1168) at $37^{\circ} \mathrm{C}$ in 5\% CO2 humid atmosphere.

\section{Blood-brain barrier permeability assay in vitro}

Permeability assays were performed in triplicate as follows: $1.5 \times 10^{5}$ endothelial cells were grown on transwell inserts (pore size: $0.4 \mathrm{~mm}$, diameter: 6.5mm, Millipore, Darmstadt, Germany) for two days, $250 \mu \mathrm{g} / \mathrm{ml}$ FITC labelled dextran FD-70 (70 kDa, Sigma) was added to the apical side of the filters and the medium in the basolateral compartment was sampled every 15 min for 2 hours. The FD-70 permeability through the cultured endothelial monolayer was determined directly by analysis of the apparent permeability coefficient (Papp).(Artursson, 1990) The concentration of FD-70 was determined with an FD-70 standard curve on plate reader (Ex. 490 nm, Em. 515 nm). Papp (cm/s) was calculated. 


\section{Oxygen Consumption}

Oxygen consumption rate (OCR) was measured at $37^{\circ} \mathrm{C}$ using an XF96e extracellular analyzer (Seahorse Bioscience, North Billerica, Massachusetts) according to the manufacturer's instructions. Briefly, the bEnd.3 cells or primary endothelial cells were seeded into Seahorse Bioscience XF96e cell culture plates $(16,000$ cells/well) in $80 \mu l$ medium and allowed to adhere and grow overnight in the $37{ }^{\circ} \mathrm{C}$ humidified incubator with $5 \% \mathrm{CO}$. Then the cells were supplied with $80 \mu 1$ medium with or without LPS (concentration varies as indicated in the text) and cultured for additional 24 hours. For the measurements of extracellular flux, the medium was changed $1 \mathrm{~h}$ prior to the start of the extracellular flux assay to un-buffered (pH 7.4) DMEM containing $2 \mathrm{mM}$ GlutaMax, $1 \mathrm{mM}$ sodium pyruvate, and $25 \mathrm{mM}$ glucose and incubated at $37{ }^{\circ} \mathrm{C}$ without CO2. Oligomycin, carbonyl cyanide 4-(trifluoromethoxy) phenylhydrazone (FCCP), rotenone and antimycin A (all from Sigma) and 10x dilutions were prepared for the assays. A sensor cartridge was loaded with $20 \mu \mathrm{l}$ of oligomycin $(10 \mu \mathrm{M})$ into Port A, $20 \mu 1$ of FCCP (30 $\mu \mathrm{M})$ into Port $\mathrm{B}, 20 \mu \mathrm{l}$ of Rotenone $(20 \mu \mathrm{M})$ and antimycin $\mathrm{A}(20 \mu \mathrm{M})$ into Port C. The Sensor cartridge was calibrated and equilibrated and then the protocol was implemented on an XF96e Analyzer. This protocol allowed determination of the basal level of oxygen consumption, the amount of oxygen consumption linked to ATP production, the maximal respiration capacity, and the non-mitochondrial oxygen consumption.

\section{Detect respiratory chain complex I-IV by flow cytometry}

We cultured cells in 6 well plates and treated cells with LPS $(1 \mu \mathrm{g} / \mathrm{ml})$ for 48 hours, then we washed cells and performed intracellular staining with an intracellular staining set (cat. 72-5775, eBioscience). We stained cells with anti-rabbit-NADH-dehydrogenase-ubiquinone-1-alpha- 
subcomplex-assembly-factor-1 (NDUFAF1, sc-292085, 1:100, Santa Crutz), anti-mouseNADH-dehydrogenase-ubiquinone-1-subunit-C2 (NDUFC2, sc-393771, 1:100, Santa Crutz), anti-mouse-NADH-dehydrogenase-ubiquinone-ironsulfur-protein-2 (NDUFS2， sc-390596, 1:100, Santa Crutz), anti-rabbit-Succinate-dehydrogenase (SDH, cat.11998, 1:100, Cell signaling, Beverly, Massachusetts), anti-rabbit-Cytochrome c (Cyc, cat.4280, 1:100, Cell signaling), anti-mouse-Cytochrome c oxidase (COX IV, cat. 4850, 1:100, Cell signaling) antibodies for $30 \mathrm{~min}$ and labeled the cells with a proper second antibody, PE-anti-rabbit (Cat.8885, 1:100, Cell signaling) or PE-anti-mouse (Cat.8887, 1:500, Cell signaling). We acquired data on BD Calibur flow cytometry and analyzed mean fluorescence intensity by Flowjo software.

\section{Immunohistochemistry staining}

To perform the immunohistochemistry co-staining of mitochondria and MyD88, the bEnd.3 cells were cultured on cover slips, stained with mitotracker (Life technologies, Grand Island, New York) for 30 minutes, washed with PBS then fixed with $2 \%$ paraformaldehyde (PFA, Polysciences, Inc.) for 10 minutes at $37^{\circ} \mathrm{C}$. The cells were blocked with $5 \%$ goat serum staining buffer and stained with anti-rabbit-MyD88 (1:200, Abcam, Cambridge, Massachusetts) overnight, then washed with PBS and stained with goat-anti-rabbit-FITC (Life technologies) for 2 hours. The cells were further washed with PBS and mounted on glass slides using prolong gold anti-fade reagent (Life technologies). The slides were photographed with confocal LSM 510 microscope Zeiss (Zeiss, Oberkochen, Germany) using software ZEN 2012. 


\section{Statistical Analysis}

Statistical analysis was performed with Prism 5 software (Graphpad software, La Jolla, California). Differences between groups were analyzed by the unpaired Student's t-test, one way ANOVA or two way ANOVA as indicated in the figure legends.

\subsection{Results}

\section{LPS increases cerebral infarction and BBB permeability in stroke}

To test the effects of a bacterial infection mimic on stroke outcome, we performed intraperitoneal (i.p.) injections of LPS $(100 \mu \mathrm{g} / \mathrm{kg}$ ) or saline 30 minutes prior to a 30 -minute transient middle cerebral artery occlusion (tMCAO) in mice (Fig. 3.1A). LPS challenge significantly increased infarct volume in the cortex $(P=0.04)$, striatum $(P=0.009)$ and total hemisphere $(\mathrm{P}=0.04)$ after 30 minute tMCAO followed by 48 hour reperfusion (Fig. 3.1B, C).

However, the time course of post-stroke BBB leakage, whether biphasic or progressive, remains subject to debate (Sandoval and Witt, 2008; Nag et al., 2011). Using Evans Blue as a tracer, our recent work demonstrated biphasic peaks of BBB openings at 6 hours and 72 hours but not at 24 hours or 48 hours reperfusion following one hour tMCAO (Hu et al., 2014). The 6-hours-peak of BBB opening may suggest the early inflammatory infiltration and brain edema in acute stroke. Thus, to determine if the acute infection challenge regulates cerebrovascular response to stroke, we compared the effects of LPS vs. saline on Evans blue extravasation at 6 hours reperfusion following tMCAO (Fig. 3.2A). Six hours after tMCAO (30 min occlusion), mice were perfused and isolated brains were divided into ipsilateral and contralateral hemispheres, and each hemisphere was analyzed separately for Evans blue extravasation. LPS significantly increased Evans blue extravasation in the ipsilateral hemisphere after tMCAO compared to control mice (Fig. 3.2B), indicating that LPS enhances cerebrovascular permeability after stroke. 


\section{LPS increases neutrophil infiltration into the injured brain in stroke}

Stroke and acute infections elicit inflammatory responses in the injured brain that are mediated by multiple factors. Neutrophils are among the first peripheral cells to infiltrate ischemic brain tissue within 30 minutes to a few hours after focal cerebral ischemia, and neutrophil infiltration is believed to contribute to acute phase damage in stroke (Gee et al., 2007). To assess brain leukocyte invasion, we measured neutrophils in brain and peripheral tissues by flow cytometry. Neutrophil accumulation in the ipsilateral and contralateral brain hemispheres was significantly greater in the LPS group compared to saline control $(\mathrm{n}=5, \mathrm{P}<0.05)$ at 6 hours tMCAO post reperfusion (Fig. 3.2C). Concurrently, fewer neutrophils and greater lymphocytes were detected in the peripheral blood and spleen but monocytes did not differ between vehicle and LPS treated mice (supplemental Figure $3.1 \mathrm{~A}, \mathrm{C}$ ). The neutrophil to lymphocyte ratio (NLR) was significantly decreased in the blood $(\mathrm{n}=5, \mathrm{P}=0.02)$, and spleen $(\mathrm{n}=5, \mathrm{P}=0.004)$ of LPStreated mice (supplemental Figure 3.1 B, D). tMCAO mice with LPS exhibited worsened neurological score at all end point experiments $(P=0.0005$, Fig. 3.2D), and mortality was higher in LPS versus saline tMCAO mice (4/23 vs. 0/18, respectively). Although neutrophil accumulation in the CNS is complex, these data are consistent with the LPS increased BBB permeability after tMCAO.

\section{LPS impairs mitochondrial oxidative phosphorylation in CVECs}

To determine if the LPS effects in stroke are due to direct effects on cerebrovascular endothelial

cells, we employed a cultured cerebrovascular endothelial cell (cCVEC) model. Immunohistochemical staining showed that CD31 (an endothelial cell marker) and TLR4 (a LPS 
receptor) colocalize in the brain vasculature (supplemental Figure $3.2 \mathrm{~A}$ ), closely resembling the expression of CD31 and TLR4 on cCVECs (supplemental Figure 3.2 B). This indicates that cCVECs have critical components for an in vitro model to investigate LPS effects on cerebrovascular endothelial cells.

The brain has a high energy demand and elevated mitochondrial content resulting in it being vulnerable to reductions in oxidative phosphorylation. Given the expression of TLR4 on the brain vascular endothelial cells, we determined if LPS would directly affect oxidative phosphorylation and mitochondrial capacity in CVECs. Using Mitotracker Red for visualization of mitochondria, we detected MyD88, an adaptor protein involved in TLR4 signaling,(Gay et al., 2014) broadly distributed but also colocalized with mitochondria in cCVECs (Fig. 3.3A), suggesting that LPS-induced signaling pathways are linked to mitochondria. To directly evaluate the mitochondrial function in cCVECs affected by LPS, a bioenergetic assay was used to examine cellular energetic oxygen consumption rates (OCR). Basal OCR did not significantly differ in cCVECs challenged by LPS for 24 hours, but maximal respiration and spare capacity were significantly reduced in cCVECs cultured with $0.1-100 \mu \mathrm{g} / \mathrm{ml}$ LPS (Fig. 3.3B \& supplemental Figure 3.3 A). Similarly, in primary cerebrovascular endothelial cells (pCVECs), an LPS challenge for 24 hours resulted in a decrease in maximal respiration and spare capacity at $100 \mu \mathrm{g} / \mathrm{ml}$ (Fig. 3.3C \& supplemental Figure 3.3B). However, reduced oxidative phosphorylation was not associated with cell death or cell viability in cCVECs or pCVECs, as evidenced by PI staining (supplemental Figure 3.4 A\&B), calcein AM staining (supplemental Figure 3.4 C\&D) for 24 hours in the exception of high dose of LPS $(100 \mu \mathrm{g} / \mathrm{ml})$. In view of the LPS effects on mitochondrial respiration, respiratory chain complex I proteins (NADH dehydrogenase ubiquinone subunits: NDUFAF1, NDUFS2 and NDUFA2), complex II protein (Succinate dehydrogenase, SDH), complex III protein (cytochrome c, Cyc), and complex IV 
protein (Cytochrome c oxidase, COX IV) were examined after a $1 \mu \mathrm{g} / \mathrm{ml}$ LPS challenge for 48 hours, and flow cytometry confirmed that LPS decreases the expression of complex I (NDUFS2 and NDUFA2), complex III (Cyc) and complex IV (COX IV) (Fig. 3.3D) proteins. Together these results strongly suggest that oxidative phosphorylation of CVECs is compromised with LPS exposure, and this effect does not induce endothelial cell death. As such, we asked if mitochondrial activity affects endothelial cell function.

\section{Impaired mitochondria disrupt CVEC tight junction and increase BBB permeability}

It is known that LPS impairs the BBB permeability both in vitro and in vivo at doses which are thought to be caused by inflammatory mediators such as cytokines, (Banks and Erickson, 2010) but little is known about the role of mitochondria in BBB integrity. Using a pharmacological strategy to manipulate mitochondrial respiration, we first demonstrated that inhibition of respiratory chain complex I with rotenone (Fig. 3.4A), uncoupling of electron flow from ATP production with FCCP (Fig. 3.4B), or inhibition of complex V with oligomycin (Fig. 3.4C) rapidly increased FITC-dextran 70 permeability in a cCVEC monolayer transwell system in vitro. Immunocytochemical analysis revealed that the normally well-defined, linear cell-cell junctions were disrupted when oxidative phosphorylation was inhibited by mitochondrial inhibitors (Fig. 3.4D). Both increased permeability and cell-cell junction disruption due to inhibition of oxidative phosphorylation occurred in the absence of cell death as evidenced by PI staining (supplemental Figure 3.5 A) and calcein AM staining (supplemental Figure $3.5 \mathrm{~B}$ ). These data suggest for the first time that mitochondria play a key role in maintaining BBB integrity in vitro. 
To determine if inhibition of mitochondria affects the BBB permeability in vivo, we developed an epidural application model for CNS drug administration in mice (Fig. 3.5A-D). This model permits local drug delivery without traumatic brain injury (Fig. 3.5C), thus allowing assessment of BBB permeability. Rotenone $(2 \mu 1,2 \mathrm{mg} / \mathrm{ml}$ dissolved in saline) was applied to the epidural surface, and BBB permeability was evaluated by Evans blue quantification after 6 hours. Mice treated with rotenone showed significantly higher Evans blue extravasation than vehicle controls (Fig. 3.5D). These data support the hypothesis that mitochondria are critical for the regulation of cerebrovascular permeability in vivo.

\section{Inhibition of mitochondrial function worsens stroke severity in mice}

If inhibition of mitochondrial function increases BBB permeability, the same perturbation would be expected to affect infarct size and stroke severity. When we treated mice with rotenone prior to tMCAO (Fig. 3.6A), increased BBB permeability (Fig. 3.6B) and infarct volumes (Fig. 3.6C), and worsened neurological deficits (Fig. 3.6D) were observed. Thus, the LPS-induced exacerbation of stroke outcome most likely is due to the deleterious effect of mitochondrial inhibition.

\subsection{Discussion}

Acute systemic infection is a risk factor and/or trigger for human stroke (Grau et al., 1995b; Grau et al., 1995a) and is associated with worsened clinical outcome (Palasik et al., 2005). Although much is known about the factors that worsen stroke severity in clinical subjects and experimental stroke models, less is understood about their mechanisms. Our data are the first to demonstrate that a bacterial infection mimic acts with ischemic challenge to markedly 
exacerbate stroke damage via a mitochondrial-dependent mechanism. This new finding could also provide an explanation for the association of infections with severity of brain damage and BBB dysfunction from stroke: the induction of hypometabolism in cerebrovascular endothelial cell mitochondria.

Mitochondrial dysfunction is increasingly recognized as an accomplice in vascular diseases and ischemic stroke. More recently, it has been shown that components of the TLR4 signaling pathways, MyD88 and TRAF6, are linked with mitochondria and affect oxidative phosphorylation (Kim et al., 2007). The expression of MyD88 in mitochondria of endothelial cells (Fig. 3.3A) supports that mitochondrial function can be affected by LPS (Fig. 3.3B\&C). It is notable that no significant effect of LPS is seen on cCVEC basal oxygen consumption and ATP-linked oxygen consumption upon treatment with oligomycin (Fig. 3.3B \& supplemental Figure 3.3), indicating that LPS does not significantly change basal oxygen demand in mitochondria after 24 hours of treatment with LPS. However, the loss of maximal respiration and spare capacity (Fig. 3.3B \& supplemental Figure 3.3) demonstrates that mitochondrial capacity is reduced by LPS and thus the effects exacerbate the mitochondrial impairment by ischemia. Cellular respiration, mitochondrial biogenesis and function, require coordinated expression of proteins encoded by nuclear and mitochondrial genes including mitochondrial complex I-IV. The reduced expression of respiratory chain complex I, III and IV proteins in cCVECs by LPS provides further evidence that LPS compromises the endothelial mitochondria function (Fig. 3.3D).

The 30 minute tMCAO model mimics a mild ischemic event that results in a small infarct (Fig. 3.1C) and does not cause visible BBB disruption at 6 hours reperfusion (Fig. 3.2B). The 
bacterial infection mimic challenge prior to tMCAO increased BBB permeability (Fig. 3.2B) and exacerbated infarct volume (Fig. 3.1C). There are inconsistent data for LPS- or cytokine-induced BBB dysfunction (Banks and Erickson, 2010) that may be due to complex mechanisms. McColl et al. (2007) demonstrated an interleukin-1-dependent mechanism of stroke exacerbation using the same LPS-tMCAO model. Others have demonstrated that LPS alters transporters or enzymes involved in BBB maintenance (Banks et al., 2008). Our observation that pharmacological inhibition of mitochondria disrupts $\mathrm{BBB}$ integrity and increases $\mathrm{BBB}$ permeability in vitro (Fig. 3.4) and in vivo (Fig. 3.5) strengthens the argument that BBB permeability is increased by LPS after stroke (Fig. 3.2) through a direct mitochondrial mechanism resulting in a larger infarct volume and worsening neurological deficit.

Increased BBB permeability, is positively correlated with massive vasogenic and cytotoxic edema post-stroke (Taheri et al., 2009; Yang and Rosenberg, 2011) and elevated neutrophils in the ischemic area that contribute to infarct expansion (Bektas et al., 2010) and poor clinical outcome (Warach and Latour, 2004). Our observation of neutropenia and low NLR may be a consequence of BBB damage that causes rapid migration of neutrophils into damaged tissue. Brain infiltration of neutrophils could also account for secondary lesion growth and increased infarct size in the ischemic brain as seen with LPS treatment (Fig. 3.1C). These data may provide translational evidence that neutropenia and low NLR may predict severe stroke.

We observed that LPS-induced inhibition of mitochondria exacerbates infarct volume and opens $\mathrm{BBB}$ in tMCAO mice (Fig. 3.1\&3.2), but that mitochondria inhibition in cCVECs and pCVECs does not affect cell viability in 24 hours (supplemental Figure 3.4). This suggests that the 
functional impairment of endothelial mitochondria by LPS may be reversible and an effective intervention could be designed to prevent/restore BBB during strokes. However, our data also indicated that a high dose of LPS $(100 \mu \mathrm{g} / \mathrm{ml})$ induced cell death and reduced cell viability in cCVECs (supplemental Figure 3.4), and extension of LPS exposure for 48 hours induced cell death in both cCVECs and pCVECs (supplemental Figure 3.4 A-D). Therefore, the effective mitochondrial directed intervention may be limited by the severity or the length of the infectious exposure to rescue BBB damage.

Leukocytosis, and cytokine and chemokine storm are features of the acute-phase reaction in systemic infections and in acute stroke. It is becoming increasingly apparent that inflammation can disturb cell energy metabolism. Our recent study found that neuronal mitochondrial function was rapidly and profoundly decreased by TNF- $\alpha$ resulting in neuronal cell death (Doll et al., 2015a). Another study demonstrated that TNF- $\alpha$ elevated oxygen consumption rate in endothelial cells (Drabarek et al., 2012). The present study did not address the complex LPSinduced effects on mitochondria, however, our novel findings refreshed the traditional idea of infection-inflammation responses and provided a new explanation for the LPS effects on BBB dysfunction: a direct mitochondrial-dependent mechanism.

The application of rotenone on the epidural surface argues that its effect on BBB opening is by compromised mitochondria in vascular endothelial cells (Fig. 3.5). Rotenone exposure is associated with clinical features of parkinsonism in humans (Tanner et al., 2011), and broadly used to induce PD like-symptoms in animal models (Chesselet and Richter, 2011) as well. This 
suggests that the mitochondrial-dependent BBB opening may not only involve in the progress of stroke but also other neurodegenerative disorders such as PD and AD.

In summary, the present study describes a previously unknown mechanism of infection-induced direct mitochondrial dysfunction in cerebrovascular endothelial cells, which compromises BBB permeability and exacerbates acute stroke outcomes. Moreover, our observations argue that maintenance of mitochondrial function is critical to the integrity of the BBB. The data also suggest a translational significance: maintenance of brain endothelial cell mitochondrial function can improve the acute outcome of stroke and perhaps other neurodegenerative diseases. 


\section{Figure Legends:}

Fig. 3.1. LPS exacerbates stroke infarct volume in mice. (A) Scheme depicting the experimental design. LPS (100 $\mu \mathrm{g} / \mathrm{kg}$, i.p.) or vehicle (saline, i.p.) was administered 30 min prior to right transient middle cerebral artery occlusion (tMCAO; $30 \mathrm{~min}$ ) and 48 hour reperfusion was performed. (B) Infarct volumes were measured at 48 hours after ischemia induction. Representative TTC-stained coronal sections used to analyze infarction of mice treated with vehicle vs. LPS. (C) Mice treated with LPS had significantly larger infarct volume than vehicle group in cortex, striatum and total hemisphere. Mean \pm S.D.; $\mathrm{n}=7$ per group; Student's t test. *, $P<0.05 ; * *, P<0.01$.

\section{Fig. 3.2. LPS increases early BBB leakage and infiltration of neutrophils into the brain of}

stroke mice. (A) Scheme of experimental design and workflow. LPS (100 $\mu \mathrm{g} / \mathrm{kg}$, i.p.) or vehicle (saline, i.p.) was administered $30 \mathrm{~min}$ prior to right tMCAO (30 min occlusion) and 6 hour reperfusion was performed. (B) Evans blue accumulation was visible in the brain of LPS pretreated stroke mice (Red arrows) and quantification of Evans Blue extravasation ( $\mu \mathrm{g} / \mathrm{g}$ brain tissue) in contralateral (left) and ipsilateral (right) hemispheres. Vehicle, $n=6 ;$ LPS, $n=7$. (C) Representative FACS data for individual hemispheres from mice treated with vehicle vs. LPS after 30 min tMCAO plus 6 hours reperfusion, and statistical analysis of infiltrated PMNs in the contralateral (left) and ipsilateral (right) hemispheres measured by FACS. $\mathrm{N}=5$ per group. (D) Neurological score at combined two end points (6 hours and 48 hours) after tMCAO. Vehicle, $n$ $=18$, LPS, $\mathrm{n}=23$. Data are expressed as mean \pm S.D.; One-way ANOVA followed by post-hoc Tukey's test was used for multiple group comparison and Student's t test was used for two group statistical analysis. (**, $P<0.01 ; * * *, P<0.001 ; * * * *, P<0.0001$. 


\section{Fig. 3.3. LPS reduces mitochondrial function in cultured and primary cerebrovascular}

endothelial cells. (A) Immunofluorescence staining of mitochondrial (MitoTracker, purple) costained with MyD88 (green), suggests that the MyD88 expresses on mitochondria of cultured cerebral vascular endothelial cells (cCVECs). Nuclei were stained with DAPI (blue). Results are representative from four independent experiments. Images were taken under $63 \times$ objective. Scale bars $=10 \mu \mathrm{m}$. (B) Bioenergetics functional assay in cCVECs exposed to various concentration of LPS compared to vehicle control for 24 hours. Basal respiration, ATP production, maximal respiration, and spare capacity were calculated from the assay presented in supplementary Figure 3.3 A. Results are representative from four independent experiments. $\mathrm{N}=$ 4 per group; **, $P<0.01$; ***, $P<0.001$; ****, $P<0.0001$ vs. vehicle group. One way ANOVA followed by post-hoc Tukey's test was used for data analysis. (C) Bioenergetics functional assay in pCVECs exposed to various concentration of LPS compared to vehicle control for 24 hours. Basal respiration, ATP production, maximal respiration, and spare capacity were calculated from the assay presented in supplementary Figure 3.3 B. Results are representative from four independent experiments. $\mathrm{N}=4$ per group; $*, P<0.05$ vs. vehicle group. One way ANOVA followed by post-hoc Tukey's test was used for data analysis. (D) Analysis of mitochondrial specific proteins for complex I proteins (NDUFAF1, NDUFS2, NDUFA2), complex II protein (succinate dehydrogenase, SDH), complex III protein (cytochrome c, Cyc), and complex IV protein (cytochrome c oxidase, cox IV) in cCVECs after a $1 \mu \mathrm{g} / \mathrm{ml}$ LPS challenge for 48 hours. Flow cytometry confirmed that LPS decreases the expression of complex I (NDUFS2 and NDUFA2), complex III (cytochrome c) and complex IV (cox IV) proteins. Results are representative from three independent experiments. $\mathrm{N}=3$ per group; *, $P<0.05 ; * *, P<0.01 ; * * * *, P<0.0001$ vs. vehicle group. Student's t test was used for data analysis. 
Fig. 3.4. Pharmacological inhibition of mitochondria increases BBB permeability in vitro. FITC-Dextran-70 permeability after addition of $20 \mu \mathrm{M}$ rotenone (A), $20 \mu \mathrm{M}$ FCCP (B) and 20 $\mu \mathrm{M}$ oligomycin (C) vs. vehicle to cultured cerebrovascular endothelial cells (cCVECs). Data are presented as both real-time rate of permeability (Two-way ANOVA followed by post-hoc Dunnett's test) and calculated apparent permeability coefficient (Papp, Student t-test). $\mathrm{N}=3$ per group. **, $P<0.01$; ***, $P<0.001$; ****, $P<0.0001$. (D) Confocal fluorescence images of cCVEC confluent monolayers after treatment with $20 \mu \mathrm{M}$ rotenone, $20 \mu \mathrm{M}$ FCCP and $20 \mu \mathrm{M}$ oligomycin vs. vehicle for 2 hours. The immunohistochemistry staining of ZO-1 (red) was performed for tight junctions. Nuclei were stained with DAPI (blue). Mitochondrial inhibitors apparently disrupted tight junctions and resulted in gaps between cells (white arrows).

Fig. 3.5. Pharmacological inhibition of mitochondria increases BBB permeability in vivo. (A) Graph depicting the epidural application (EA) model. (B) Exposure of epidural membrane: vessels were visible under surgical microscope. (C) TTC staining in the EA model showing no brain injury. (D) Evans blue accumulation was visible in the brain of rotenone applied mice (red arrows) in EA model and quantitative analysis of Evans blue extravasation ( $\mu \mathrm{g} / \mathrm{g}$ brain tissue) in individual brain. $\mathrm{N}=5$ per group; One-way ANOVA followed by post-hoc Tukey's test. Data are expressed as mean \pm S.D.; **, $\mathrm{P}<0.01$.

Fig. 3.6. Impairment of mitochondria function exacerbates stroke outcomes. (A) Scheme of experimental design and workflow. Rotenone $(1 \mathrm{mg} / \mathrm{kg}$, i.p.) or vehicle (saline, i.p.) was administered 30 min prior to right tMCAO (60 min occlusion), BBB permeability was evaluated at 6 hour reperfusion and infarct volume was measured at 24 hour reperfusion. (B) Representative brain coronal sections for Evans blue accumulation and quantitative analysis of Evans Blue extravasation ( $\mu \mathrm{g} / \mathrm{g}$ brain tissue) in contralateral (left) and ipsilateral (right) hemispheres. Vehicle, $\mathrm{n}=4$; Rotenone, $\mathrm{n}=4$. One-way Data are analyzed with ANOVA 
followed by post-hoc Tukey's test $(*, P<0.05)$. (C) Representative TTC-stained coronal sections used to analyze infarct of tMCAO mice and quantitative analysis of infarct volumes. Mice treated with rotenone had significant larger infarct volume than vehicle group, in cortex, striatum, and total hemisphere. Rotenone (1 $\mathrm{mg} / \mathrm{kg}$, i.p.) or vehicle (saline, i.p.) was administered 30 min prior to right tMCAO (1 hour occlusion) and 24 hour reperfusion was performed. Vehicle, $\mathrm{n}=8$; Rotenone, $\mathrm{n}=8$. Data are analyzed with Student's t tests $(* *, P<$ 0.01 ; ***, $P<0.001$; ****, $P<0.0001$.). (D) Rotenone worsened neurological deficits at 6 and 24 hours after tMCAO. Vehicle, $\mathrm{n}=12$; Rotenone, $\mathrm{n}=12$. Data are analyzed with Student's $\mathrm{t}$ test $(* * * *, P<0.0001)$. 


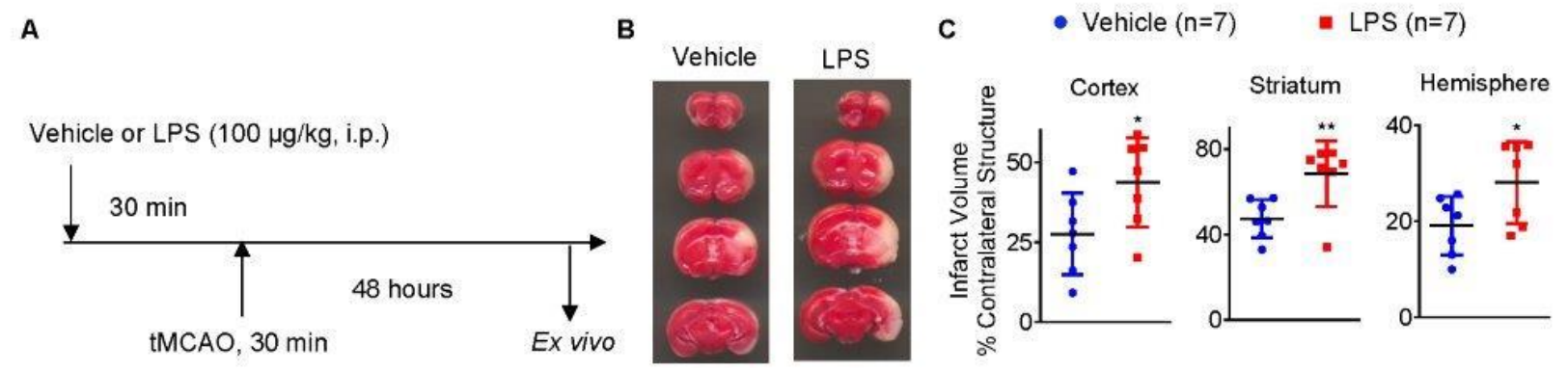

Figure 3.1 
A

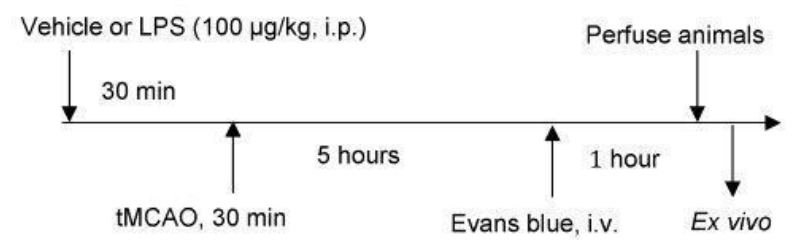

C
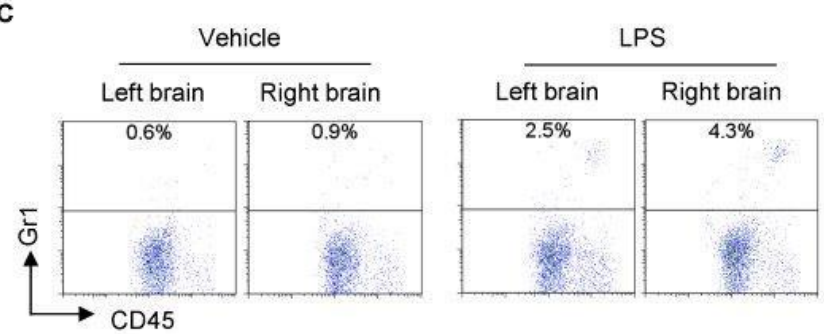

B
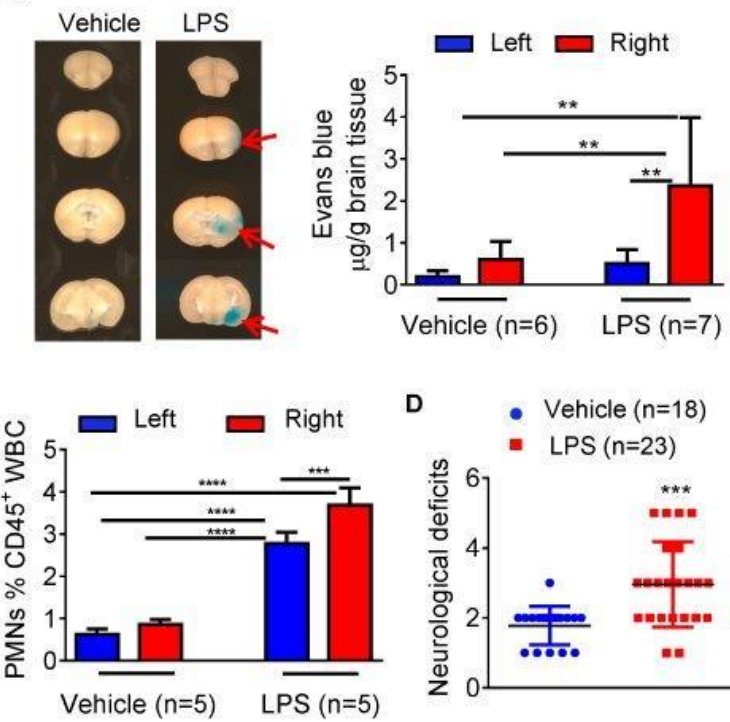

Vehicle $(n=5) \quad$ LPS $(n=5)$

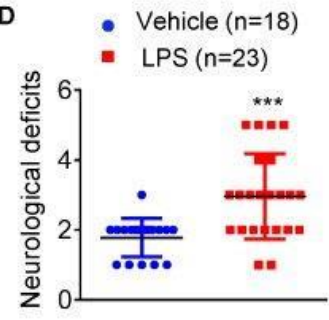

Figure 3.2 


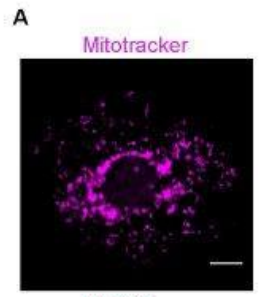

MyD88
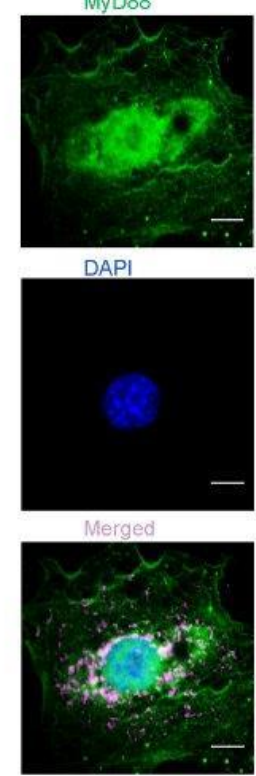
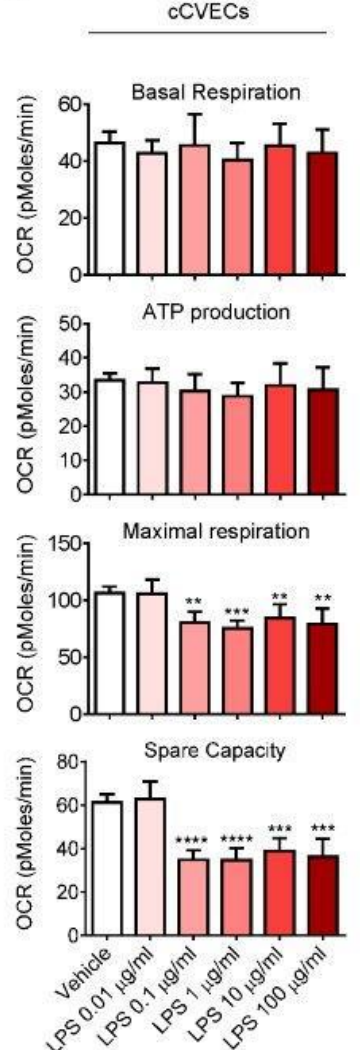

c
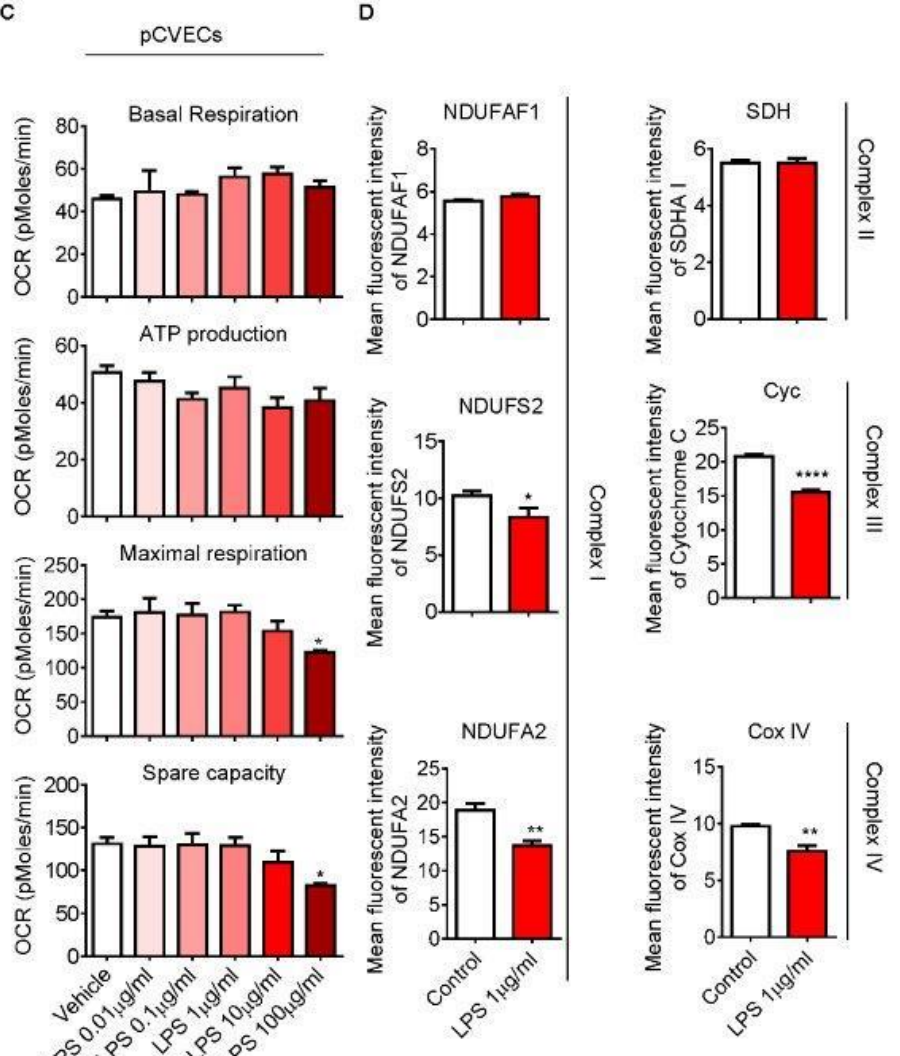

Figure 3.3 
A

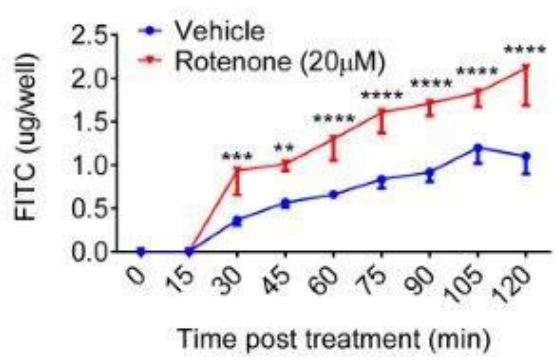

B

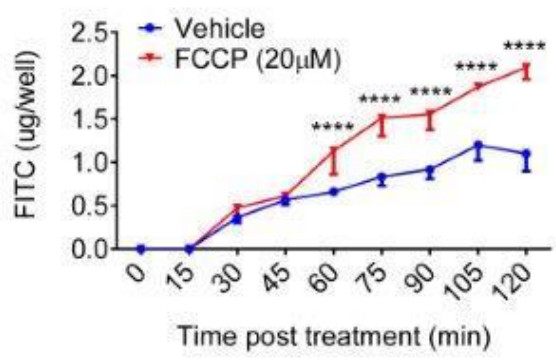

C

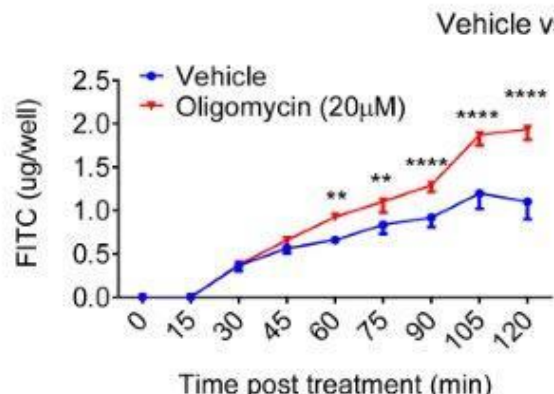

D

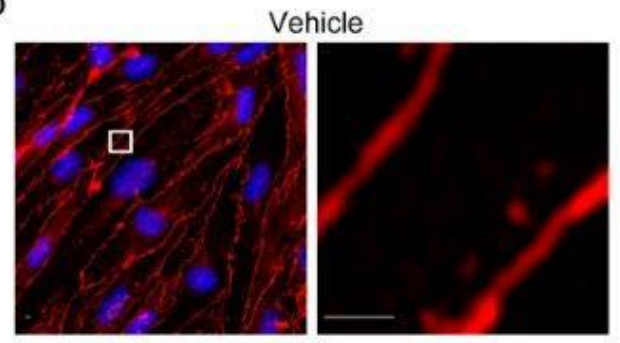

Rotenone

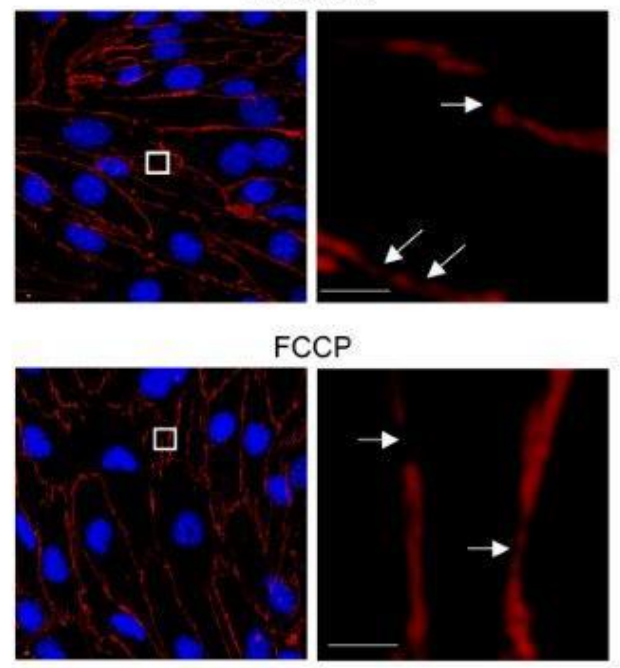

Oligomycin
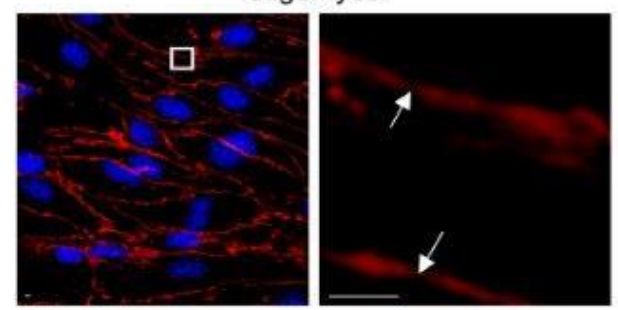

Figure 3.4 
A

Scheme for EA model

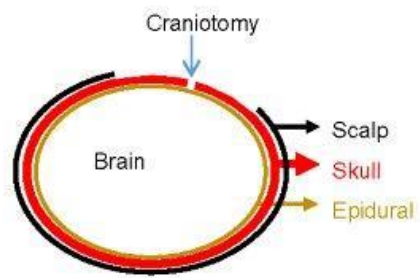

B

Exposure of epidural membrane

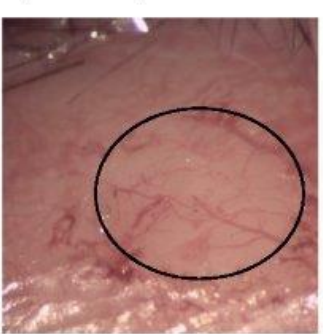

c

TTC staining for EA model

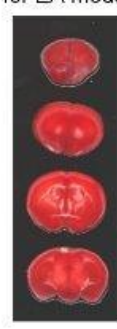

D

Vehicle Rotenone

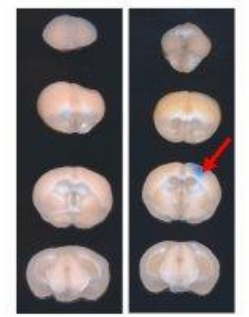

- Vehicle $(n=5)$

- Rotenone $(n=5)$

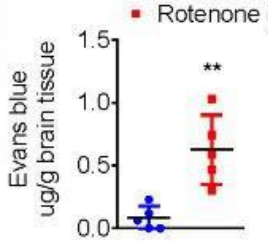

Figure 3.5 
A

B
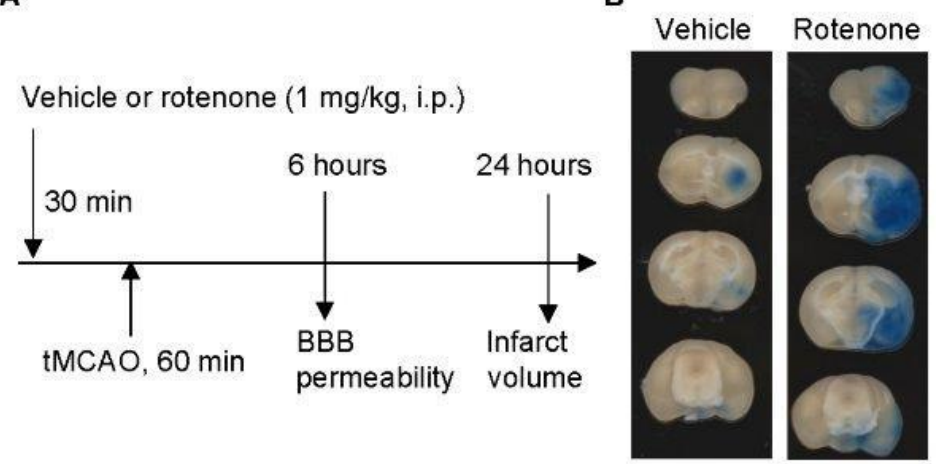

- Vehicle $(n=4)$

- Rotenone $(n=4)$

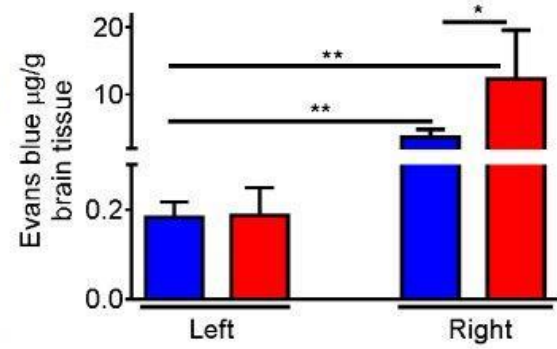

C

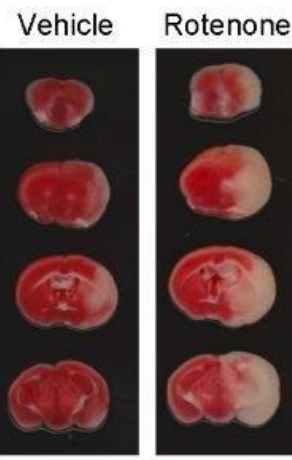

- Vehicle $(n=8)$

- Rotenone $(\mathrm{n}=8)$

D

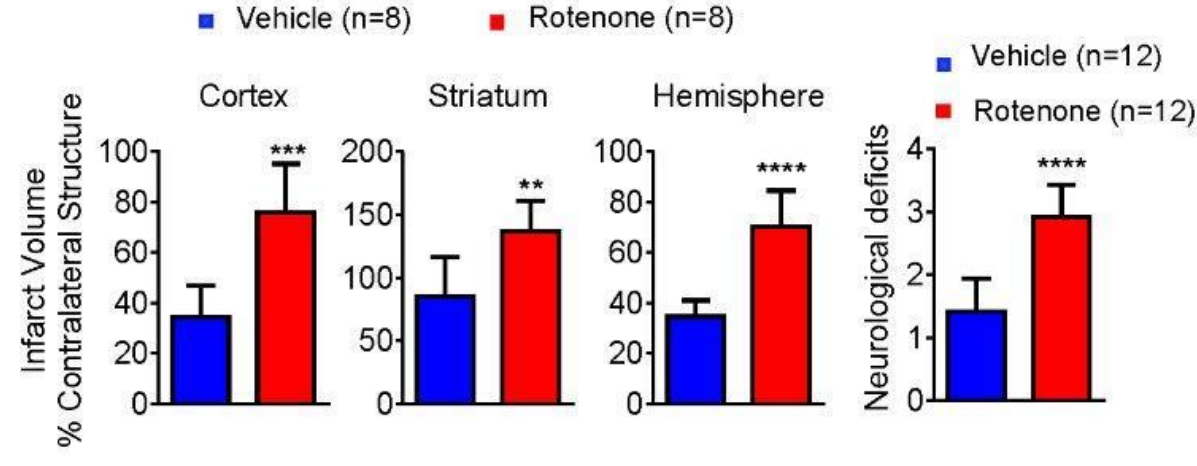

Figure 3.6 


\section{Supplemental Materials and Methods:}

\section{Cell viability assay}

We performed the cell viability assay simultaneously with the oxygen consumption assay. We seeded cells in 96 black well plates and treated with LPS. We added PI $(2 \mu \mathrm{g} / \mathrm{ml})$ to evaluate cell death on plate reader (Ex. $535 \mathrm{~nm}$ and Em. $617 \mathrm{~nm}$ ), washed with PBS, added Calcein AM (Life technology) and stained for 30 minutes to measure cell viability (Ex. $490 \mathrm{~nm}$ and Em. 520 $\mathrm{nm})$.

\section{Immunohistochemistry staining}

To obtain brain samples for immunohistochemistry assays, animals were anesthetized with isofluorane and perfused with PBS followed by $10 \%$ formalin PBS. Brains were removed, postfixed overnight in $10 \%$ formalin PBS at $4^{\circ} \mathrm{C}$, cryoprotected in graded sucrose solutions, and embedded in Tissue-Tek OCT (VWR Scientific). Coronal sections $(20 \mu \mathrm{m})$ were cut through, blocked with 5\% goat serum staining buffer and stained with rabbit anti-mouse TLR4 (1:100, Abcam, Cambridge, Massachusetts) and rat anti-mouse CD31 (1:200, Abcam) overnight, and then washed with PBS and stained with goat-anti-rabbit-FITC and goat-anti-rat-PE antibodies (Life technologies, Grand Island, New York) for 2 hours. The sections were further washed with PBS and mounted on glass slides using prolong gold anti-fade reagent (Life technologies).

To perform the immunohistochemistry staining on bEnd.3 cells, the cells were cultured on cover slips and fixed with 4\% cold paraformaldehyde (PFA, Polysciences, Inc.) followed by blocking, staining, washing and mounting, as outlined above. The slides or sections were photographed with confocal LSM 510 microscope Zeiss (Zeiss, Oberkochen, Germany) using software ZEN 2012. 


\section{Statistical Analysis}

Statistical analysis was performed with Prism 5 software (Graphpad software, La Jolla,

California). Differences between groups were analyzed by the unpaired Student's t-test or one way ANOVA as indicated in the figure legends. 


\section{Supplemental Figures:}

A.

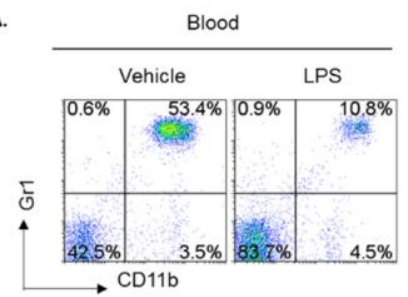

c.

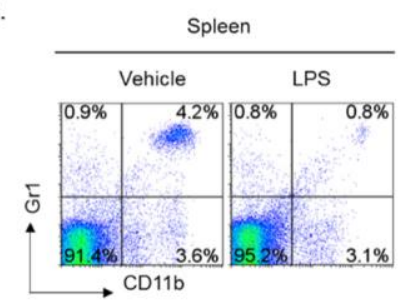

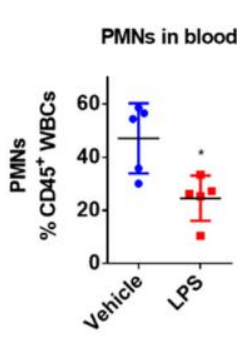
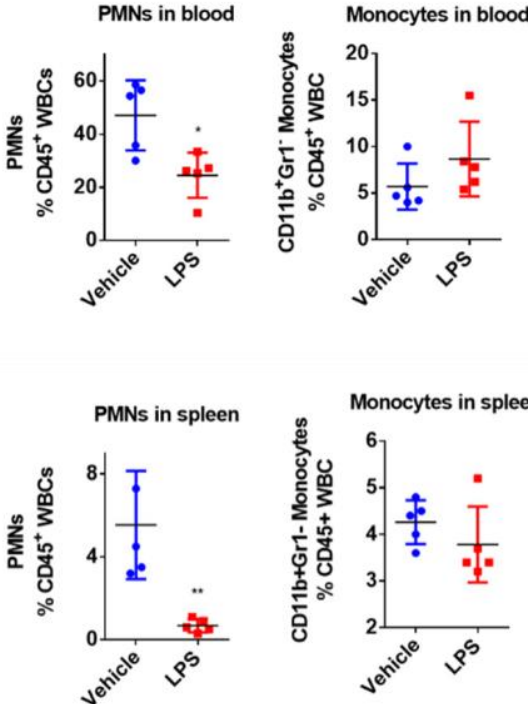

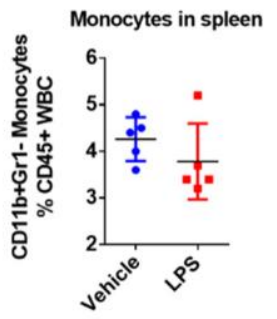

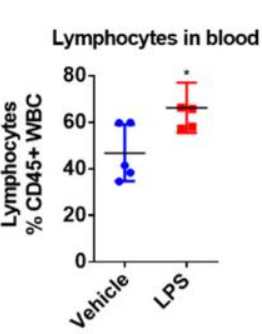
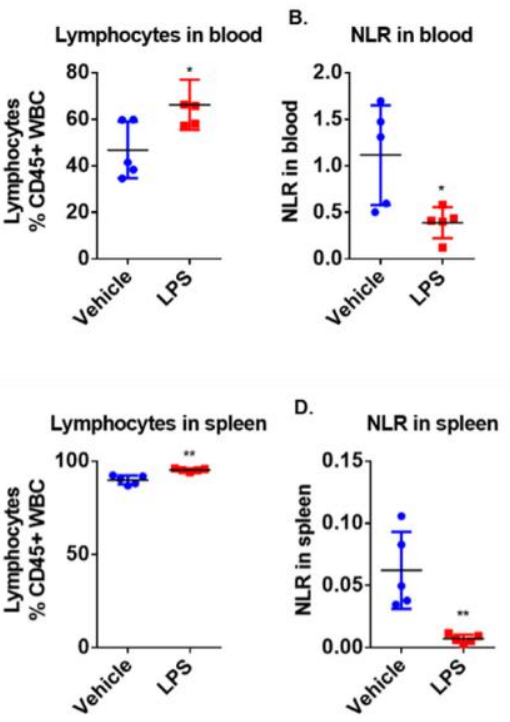

Supplemental Figure 3.1. LPS significantly decreases neutrophils and increases lymphocytes in peripheral blood and spleen after stroke. Analysis of neutrophils, monocytes and lymphocytes in blood (A) and spleen (C). Representative FACS data of blood and spleen showing percentage of Gr1+ polymorphonuclear neutrophils (PMNs), CD11b+Gr1monocytes, and CD11b- Gr1- lymphocytes gated in CD45+ white blood cells (WBCs). Neutrophil-to-lymphocyte ratio (NLR) in blood (B) and spleen (D). Mice pre-treated with LPS had significantly fewer neutrophils but more lymphocytes in the blood and spleen than vehicle group after 30 min tMCAO and 6 hours reperfusion. Data are expressed as mean \pm S.D.; $n=5$ per group; Student t-test was used for significant analysis, * $P<0.05 ; * *, P<0.01$. 
A

B
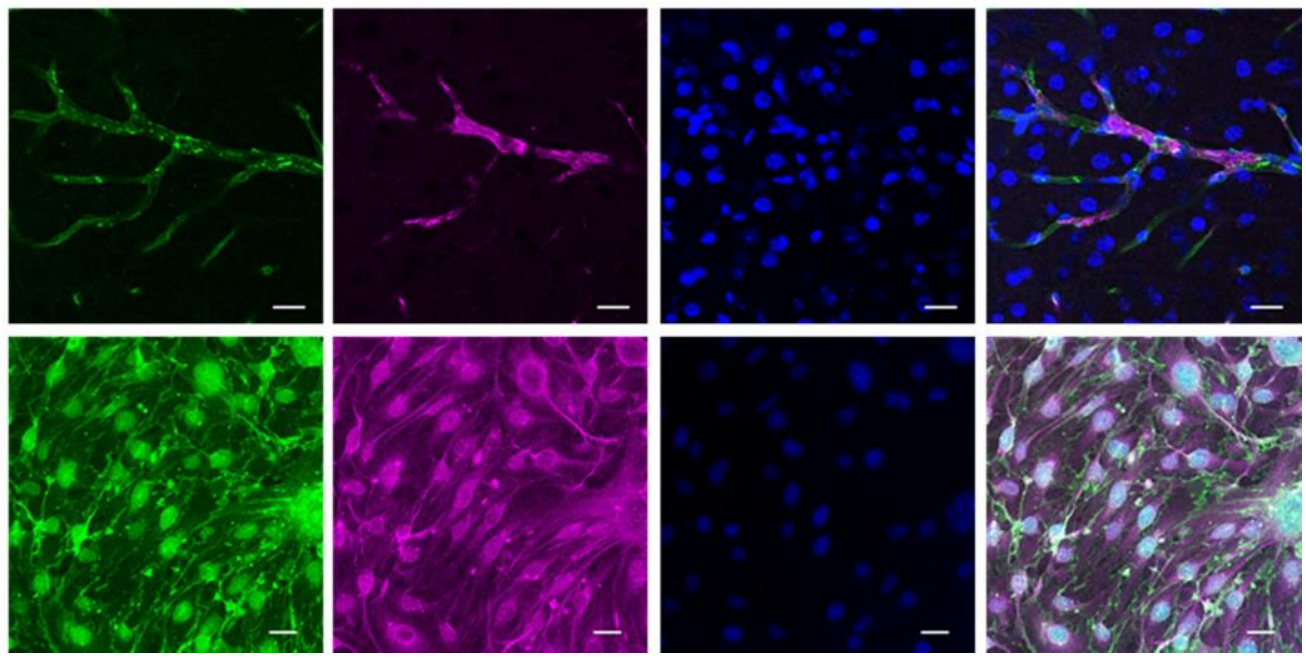

Supplemental Figure 3.2. LPS receptor, TLR4, is expressed by endothelial cells in brain tissue and in culture. (A) Sections from normal mouse brains stained with antibodies to TLR4 (purple) show TLR4 staining in association with CD31 (green), a specific marker for endothelial cells. (B) Micrographs showing TLR4 (purple) expression in cultured cerebrovascular endothelial cells (CCVECs, bEnd.3 cell line). Nuclei were stained with DAPI (blue). Results are representative from four independent experiments. Images were taken under $20 \times$ objective. Scale bars, $20 \mu \mathrm{m}$. 
A.

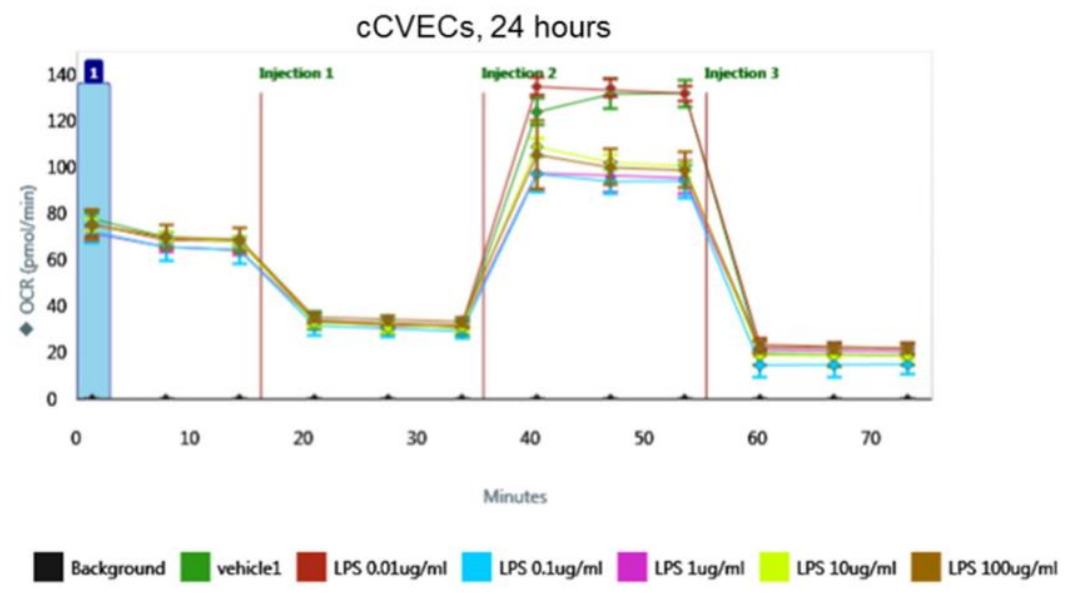

B. pCVECs, 24 hours

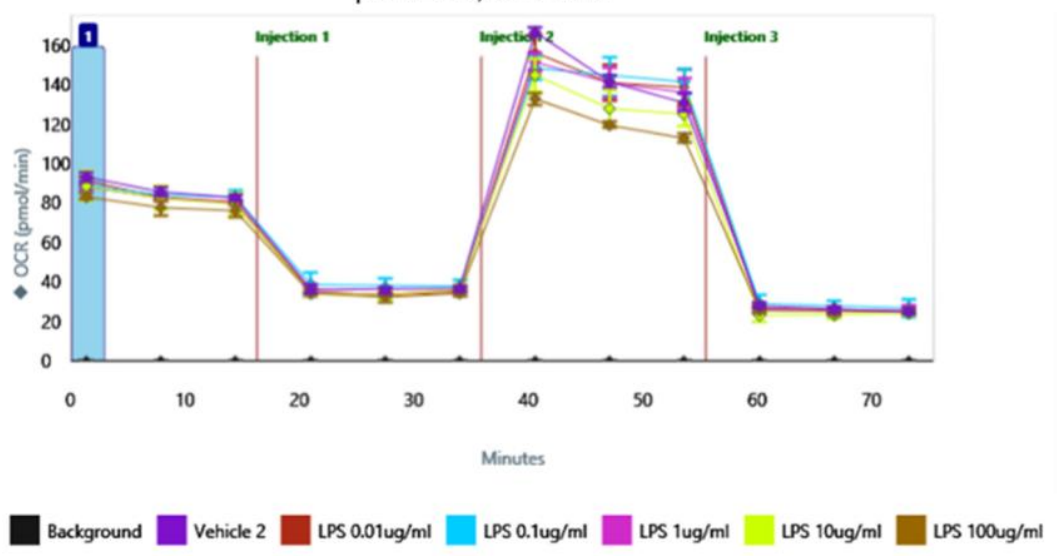

Supplemental Figure 3.3. The raw data of oxygen consumption rate determined by the Seahorse XF96e analyzer. (A) cultured cerebrovascular endothelial cells (cCVECs, bEnd.3 cell line) were incubated with various concentrations of LPS for 24 hours then oxygen consumption rate (OCR) was determined by the Seahorse XF96e analyzer. (B) Primary cerebrovascular endothelial cells (pCVECs, originally from adult mouse brain) were incubated with various concentrations of LPS for 24 hours then OCR was determined by the Seahorse XF96e analyzer. The mitochondrial inhibitors: oligomycin, FCCP, and rotenone plus antimycin A were sequentially injected after measurement points 3, 6, and 9 as indicated. Data are presented as mean \pm S.D. at each measurement. 

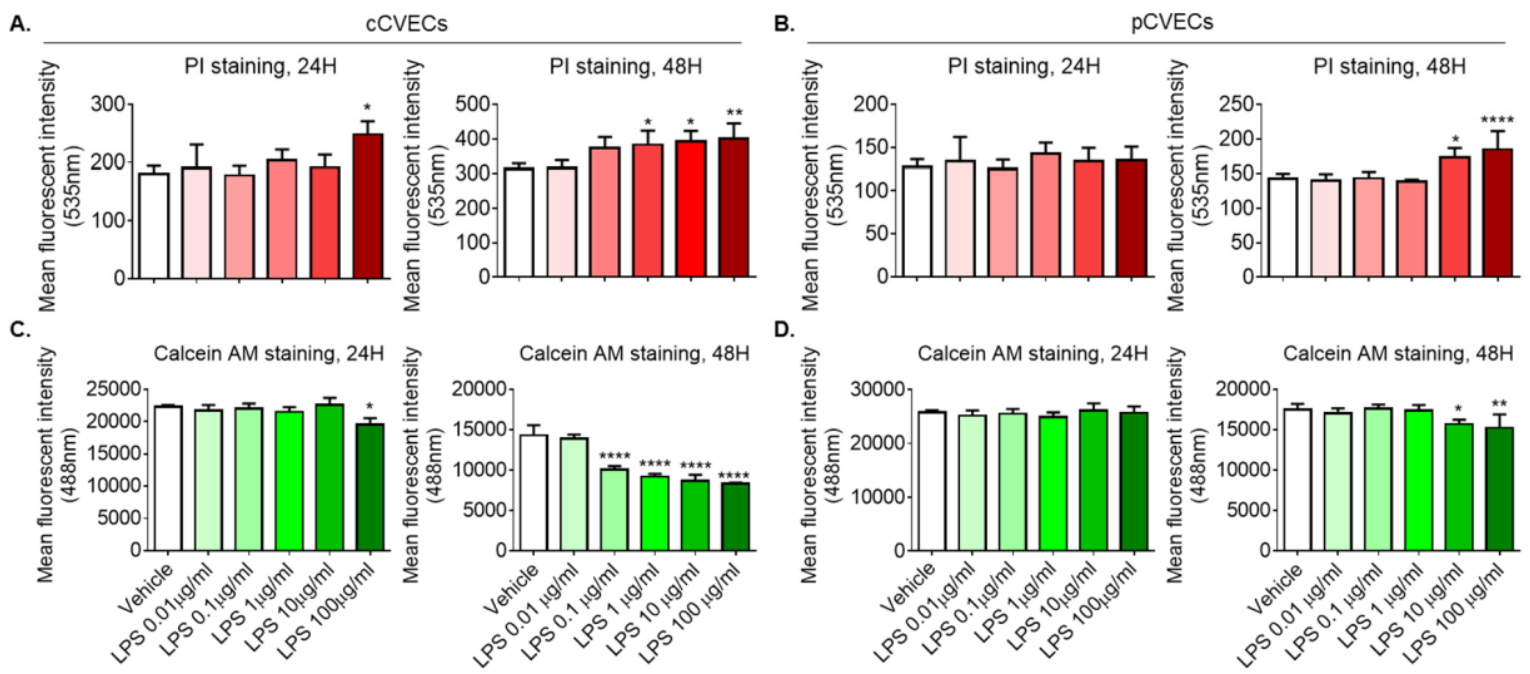

Supplemental Figure 3.4. LPS and cell viability in cultured cerebrovascular endothelial cells and primary cerebrovascular endothelial cells. Evaluation of LPS on cell death by propidium iodide (PI) staining. cCVECs (A) or pCVECs (B) were cultured with LPS (0.01 $100 \mu \mathrm{g} / \mathrm{ml})$ for 24 and 48 hours then stained with PI $(2 \mu \mathrm{g} / \mathrm{ml})$. A microplate reader was immediately used to determine the mean fluorescent intensity of PI staining. Effect of LPS on cell viability of cCVECs (C) or pCVECs (D) by calcein-AM staining. After PI staining, cells were washed with PBS and incubated with calcein-AM $(5 \mu \mathrm{M})$ for 30 minutes. A microplate reader was used to determine the mean fluorescent intensity of Calcein AM staining. Data are presented as mean \pm S.D., analyzed by One-way ANOVA followed by post-hoc Tukey's test. LPS at $100 \mu \mathrm{g} / \mathrm{ml}$ significantly induced cell death and reduced cell viability in cCVECs after 24 hours culture. *, $P<0.05, * *, P<0.01$;

****, $P<0.0001$. 

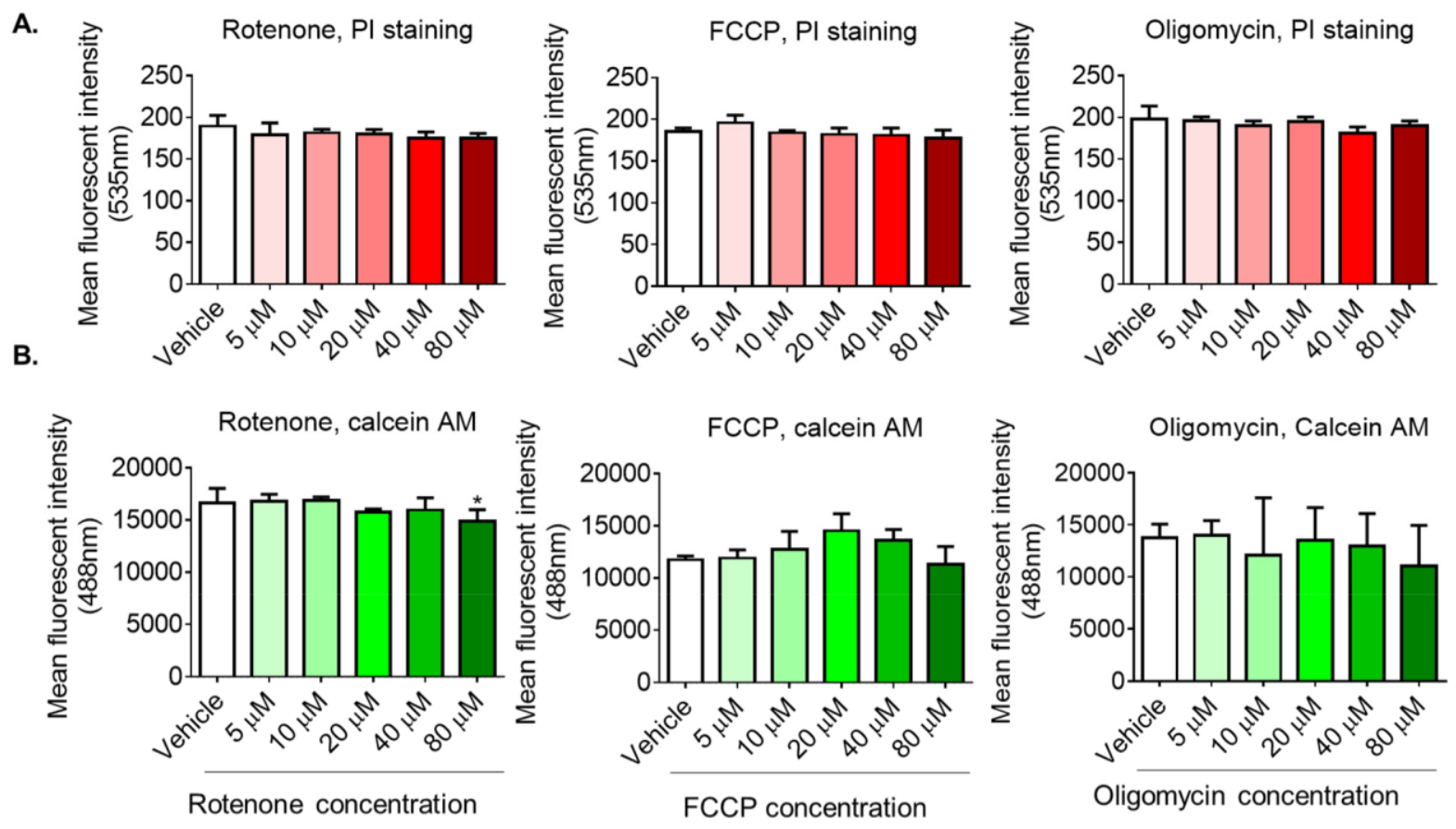

Supplemental Figure 3.5. Mitochondria inhibitors and cell viability in cultured cerebrovascular endothelial cells at 2 hours. (A) Evaluation of mitochondria inhibitors: rotenone, FCCP and oligomycin on cell death by propidium iodide (PI) staining in cCVECs. Confluent cCVEC monolayer were treated with rotenone, FCCP or ologomycin $(5-80 \mu \mathrm{M})$ for 2 hours then stained with PI $(2 \mu \mathrm{g} / \mathrm{ml})$. A microplate reader was immediately used to determine the mean fluorescent intensity of PI staining. (B) Effects of mitochondria inhibitors on cell viability by calcein-AM staining. After PI staining, cells were washed with PBS and incubated with calcein- AM $(5 \mu \mathrm{M})$ for 30 minutes. Mean fluorescence intensity was determined using a microplate reader. Data are presented as mean \pm S.D., analyzed by One-way ANOVA followed by post-hoc Tukey's test. Rotenone at $80 \mu \mathrm{M}$ significantly reduced cell viability after 2 hours treatment. Data are presented as mean \pm S.D., analyzed by One-way ANOVA followed by posthoc Tukey's test.

*, $P<0.05$. 


\section{Chapter 4}

\section{Lipopolysaccharide Exacerbates Infarct Size and Results in Worsened Post-Stroke Behavioral Outcomes}

Danielle N. Doll, Elizabeth B. Engler-Chiurazzi, Sara E. Lewis, Heng Hu, Ashley E. Kerr, Xuefang Ren, and James W. Simpkins

Doll DN, Engler-Chiurazzi EB, Lewis SE, Hu H, Kerr AE, Ren X, Simpkins JW. (2015) Lipopolysaccharide Exacerbates Infarct Size and Results in Worsened Post-Stroke Behavioral Outcomes. Behavioral and Brain Functions, Under Review. 


\subsection{Abstract}

Background: A third of ischemic stroke cases have no traditional underlying causes such as hypertension, diabetes, atherosclerosis, obesity, or age. Moreover, thirty to forty percent of strokes occur during or acutely after an active infection and the incidence of stroke increases during flu season. We and others have shown that the combination of a minor bacterial infection mimic, $100 \mu \mathrm{g} / \mathrm{kg}$ of lipopolysaccharide (LPS) prior to a minor stroke - 30 minute transient middle cerebral artery occlusion (tMCAO) - exacerbates infarct volume in a mouse model. Thus, experimental and epidemiological data strongly suggest that infection and/or inflammation play a role in stroke occurrence and severity. However, to date, long-term outcomes of stroke during an active infection has not been studied.

Methods: 3-4 month old C57B16/J mice were treated with saline or LPS 30 minutes prior to a 30 minute tMCAO or sham surgery. A behavioral battery was administered to assess health status/sickness behavior, neurological deficits, motor, cognitive, and affective behaviors.

Results: We show for the first time that exposure to a low dose of LPS prior to a mild stroke significantly worsens neurological deficits and sickness scores. Motor, cognitive, and affective behaviors were assessed post-stroke and while stroke significantly affected motor behavior on rotarod, LPS did not increase the motor deficits. We did not observe any effects of stroke or LPS on cognitive and affective behaviors.

Conclusions: Our observations of the association between infection, stroke, and worse sickness and neurological outcomes identify 1) a clinical need to aggressively treat infections in people with risk factors for stroke and 2) the need to understand the mechanism(s) of the association between infections and stroke. 


\subsection{Introduction}

Stroke is the $5^{\text {th }}$ leading cause of death in the United States and the leading cause of disability (Lackland et al., 2014). Numerous risk factors such as diabetes, hypertension, and atherosclerosis have been recognized to increase susceptibility to stroke; however, the epidemiology of ischemic stroke is not sufficiently explained by the prevalence of these cerebrovascular risk factors (Grau et al., 1995b). In at least a third of ischemic stroke cases, the patients lack traditional risk factors and have no apparent cause. The presence of systemic infection could be an important mediating risk factor. Indeed, thirty to forty percent of strokes occur during or acutely after an infection (Grau et al., 1995b) suggest an association between systemic infection and stroke. Although infection has been implicated in increasing susceptibility to stroke, few studies have examined how an infection prior to stroke affects outcome. McColl et al. (2007) and Doll et al. (2015c) found that administering lipopolysaccharide (LPS) 30 minutes prior to transient middle cerebral artery occlusion (tMCAO) resulted in an increased infarct volume. We have shown that a mechanism by which lipopolysaccharide (LPS) exacerbates stroke damage involves an increase in blood brain barrier (BBB) damage through mitochondrial dysfunction (Doll et al., 2015c) and others have suggested a cytokine-dependent mechanism (McColl et al., 2007). Together, these findings suggest an association between systemic infection and stroke.

While it is clear that bacterial infection prior to stroke can affect acute stroke damage (McColl et al., 2007; Doll et al., 2015b), to our knowledge no studies have examined the long-term effects of a bacterial infection mimic on behavioral outcomes. We hypothesized that LPS administered prior to stroke would exacerbate the motor, cognitive, and affective behavioral deficits post- 
stroke. To test our hypothesis, we used an animal model of mild infection and mild stroke developed by McColl et al. (2007), then assessed sickness behavior, neurological deficits, locomotor, cognitive, and affective behaviors to determine if a bacterial infection can exacerbate detrimental post-stroke functional outcomes.

\subsection{Methods}

Subjects

C57/BL6J male mice (3-4 months old, 25-30g, Jackson Laboratories) were used for all studies. All procedures were conducted according to criteria approved by the Institutional Animal Care and Use Committees at the West Virginia University.

\section{Randomization and blinding of the animal experiments}

To assign groups (Vehicle Sham, LPS Sham, Vehicle Stroke, LPS Stroke), we numbered the animals and applied a simple randomization by using excel-generated random numbers. To avoid biases, we also assured that sham and stroke surgeries along with the different treatments (LPS or vehicle) were performed on the same day. The experimenters were blinded to the pretreatments and data analysis.

\section{Drug administration}

LPS (Escherichia coli 055:B5, $100 \mu \mathrm{g} / \mathrm{kg}$, Sigma) dissolved in saline (B. Braun Medical Inc. Irvine, California) was administered via an intraperitoneal injection 30 min prior to tMCAO or sham surgery. An equal volume of saline was administered to vehicle treated mice. 


\section{Ischemic model and sham surgery}

We performed focal cerebral ischemia for $30 \mathrm{~min}$ by occlusion of the right middle cerebral artery with a 6.0 monofilament suture (Doccol, Sharon, Massachusetts). All surgical anesthesia was induced with $4-5 \%$ isoflurane until the animal showed no response to a toe pinch, and was maintained with $1-2 \%$ isoflurane via face-mask in $\mathrm{O}_{2}$-enriched air. We used laser Doppler flowmetry (Moor instruments, United Kingdom) to detect regional cerebral blood flow and confirm a successful occlusion ( $>70 \%$ decrease in flow). Rectal body temperature was maintained at $37 \pm 0.5{ }^{\circ} \mathrm{C}$ during surgery. One cohort of mice was euthanized at 48 hours poststroke to assess infarct area, and a separate cohort was euthanized at the end of the behavioral battery.

\section{Analysis of brain infarct area-Cohort 1}

In one cohort, a total of 28 mice ( $\mathrm{N}=7$ /group) were euthanized with isoflurane 48 hours postischemia. We removed the brains and cut $2 \mathrm{~mm}$ coronal sections with a mouse brain matrix. We stained the sections with $2 \%$ 2,3,5-triphenyltetrazolium chloride (TTC, Sigma, Saint Louis, Missouri) in phosphate buffer solution (PBS) at $37^{\circ} \mathrm{C}$ for 30 minutes then fixed the tissue in $10 \%$

formalin phosphate buffer for digital photograph. We analyzed the digitized image of each brain section using computerized image analysis software (ImageJ, NIH) in a double-blinded manner. Additional data from this cohort of animals was published in Doll et al. (2015c). 


\section{Behavioral Test Battery-Cohort 2}

In another cohort, a total of 40 mice (Vehicle Sham $=10 ;$ LPS Sham $=10$; Vehicle Stroke $=10$;

LPS Stroke $=10$ ) underwent behavioral testing. Two animals were excluded from the LPS stroke group; one due to post-mortem evidence of cerebral hemorrhage, and a second due to a surgery complication. The animal excluded due to a surgery complication was replaced resulting in an $n$ $=9$ for the LPS stroke group. The animals were treated and assessed in two cohorts with $n=5$ in each group for each cohort. We selected the following behavioral tests to assess a range of affective, locomotor, and cognitive abilities and to identify and control for potential locomotor, visual, or motivational alterations effected by stroke and bacterial infection prior to stroke. Figure 4.1 depicts the time-course of behavioral testing. All animals underwent the entire test battery with the exception of Morris water maze. The first cohort showed no significant effects or trends toward a treatment or surgery effect on this test; thus, we did not subject the second cohort of animals to Morris water maze testing. However, the timing post-stroke of the other behavioral assessments were maintained constant across cohorts. All testing was completed during the light cycle (08:00-15:00) by the same experimenter, who was blind to treatment group status. Central air conditioning fans provided white noise in the testing room. Between each animal, the behavior apparatus was cleaned of debris with Virkon and, when appropriate (Open Field, Elevated Plus Maze), 50\% ethanol.

\section{Health and Sickness Behavior Screen}

Overall health and sickness behavior was assesses using an objective 20 point screen developed in our laboratory. The health screen was administered daily by the same experimenter who was blind to treatment group status. The screen encompasses seven physical domains designed to 
provide insight into the global physical health of an animal. The screen was designed to be rapid and easy to administer, to be minimally invasive, and to ensure consistency in scoring across the post-stroke recovery period. To assess health status and sickness behavior, the animal was observed in its home cage for general appearance, posture, respiration, and spontaneous locomotion/social interaction. Body condition (emaciation and hydration) was assessed by the

pinch test. Body temperature and body weight were also measured (see supplemental Table 4.1 for scoring criteria). The screen was administered twice prior to tMCAO and each day posttMCAO for 7 days between 0800 and 0930 .

\section{Modified Neurological Severity Score (mNSS)}

The mNSS, adapted from Chen et al. (2005), assessed neurologic function on a scale of 0 to 12 with 0 being normal and 12 being maximal deficit (see supplemental Table 4.2 for scoring criteria). The mNSS used here was composed of motor and balance tests; the reflex test was eliminated due to habituation of the mice after repeat testing. mNSS scores were determined twice prior to tMCAO and each day post-tMCAO for 7 days between 0800 and 0930 .

\section{Cylinder Test}

The cylinder test evaluates spontaneous forelimb use and asymmetries resulting from neural injury(Schaar et al., 2010). The animal was placed in a transparent cylinder $(12 \mathrm{~cm}$ wide, $19 \mathrm{~cm}$ tall) for 5 minutes and allowed to freely explore. Performance was video recorded for later assessment. The number of independent wall placements for the right and left forelimbs, in addition to placement of both forelimbs simultaneously, were recorded. Cylinder test 
performance was conducted twice prior to tMCAO to account for pre-operative handedness biases, as well as 1, 3, 5 and 7 days post-tMCAO.

\section{Open Field}

This task evaluates locomotor activity and emotional reactivity (Denenberg, 1969). A white plastic box $(60 \mathrm{~cm}$ x $60 \mathrm{~cm}$ x $15 \mathrm{~cm})$ was placed upon a table. Five desk lamps (60 watt each) provided indirect illumination. At the start of the trial, each mouse was placed in arena at the midpoint of the South wall and received a 6 minutes session. Locomotor activity was recorded using Ethovision 8.5 (Noldus Information Technology, Wageningenm, Netherlands). For analysis, the box floor was divided into inner zone, middle frame, and perimeter zones. The dependent variables were fecal boli excreted, and distance moved $(\mathrm{cm})$ in the arena as well as distance moved $(\mathrm{cm})$ in each zone.

\section{Elevated Plus Maze}

This task assesses anxiety-like behavior in a novel environment, with mice spending less time in open arms considered 'anxious'(Hogg, 1996). Two intersecting arm pairs were arranged in a 'plus' configuration and elevated $60 \mathrm{~cm}$ from the floor. One arm pair, $58 \mathrm{~cm}$ long x $5 \mathrm{~cm}$ wide, was enclosed by walls $15 \mathrm{~cm}$ tall and the other arm pair remained open (no walls or edges). Room illumination consisted of ambient lighting. Each mouse was placed at the arm intersection

point and allowed to freely explore for 5 minutes. The dependent variables were the frequency of entries into each arm pair, the time spent (s) in the open arm pair, and the number of fecal boli excreted. 


\section{Visible Platform}

This task confirms visual and motor competence for water maze testing (Engler-Chiurazzi et al., 2012). A circular tub (140 cm in diameter) was filled with clear water. A white- and blackcolored platform with a white/black flag was positioned approximately $0.5 \mathrm{~cm}$ above the water surface in a fixed location within the pool. Blue curtains covered obvious extra maze cues. The drop-off location varied semi-randomly across trials. Each mouse had $90 \mathrm{sec}$ to locate the platform, where it remained for $15 \mathrm{sec}$ before being placed back into its heated cage. If an animal did not locate the platform within the allotted time limit, it was gently guided to the platform. Between animals, the maze was cleaned of debris and olfactory cues were disrupted using a fishnet. The inter-trial interval was approximately $12 \mathrm{~min}$. Latency $(\mathrm{sec})$ to reach the platform was the dependent measure.

\section{Morris Water Maze}

Acquisition: The Morris water maze tests hippocampal-dependent spatial reference memory (Morris et al., 1982), memory for information that remains consistent across time (Olton, 1979). A round tub (140 $\mathrm{cm}$ in diameter) was filled with room temperature water made opaque with non-toxic paint. Spatial cues (shelving, door, striped posters, etc) were indirectly lit. Briefly, the mouse was placed in the maze from any of four locations (North, South, East, or West) and had $60 \mathrm{sec}$ to locate a hidden platform submerged $1 \mathrm{~cm}$, which remained in a fixed location (Northwest quadrant; NW). If an animal failed to locate the platform, it was gently guided to the platform. After $15 \mathrm{sec}$ on the platform, the mouse was placed into its heated cage until the next trial. Between animals, the maze was cleaned of debris and olfactory cues. There was approximately a 15 min inter-trial interval between trials. A tracking system (Ethovision XT 8.5) 
analyzed each mouse's swim path. Animals received 4 trials for 6 days. The dependent measure was swim distance $(\mathrm{cm})$. Swim speed $(\mathrm{cm} / \mathrm{s})$ was assessed to determine possible locomotor differences.

Probe Trial: To assess platform localization, on the 6th day of testing an additional probe trial was given (trial 5), whereby the platform was removed from the maze. Percent of total swim distance $(\mathrm{cm})$ in the target NW quadrant (i.e., quadrant that once contained the platform) versus the opposite Southeast (SE) quadrant and frequency of platform zone crossings were the dependent measure (Stavnezer et al., 2002).

Reversal: To assess cognitive flexibility/perseveration (D'Hooge and De Deyn, 2001), reversal learning was assessed the following day, whereby the platform was relocated to the SE quadrant. Animals received 4 trials for 2 days, with a 15 min delay between trials three and four. Trial 5 was a probe trial on day 2. All testing procedures were identical to the Morris water maze acquisition and probe testing.

\section{Accelerating Rotarod}

This task assesses locomotor performance and coordination. A textured plastic horizontal rod (3 $\mathrm{cm}$ in diameter) was mounted $14.5 \mathrm{~cm}$ above a pressure-sensitive base (Ugo Basile). Acceleration was set to 4-44 rpm in $300 \mathrm{sec}$ (Jung et al., 2011). For a given trial, a mouse was placed on the rod and the motor and timer switch were activated. Acceleration continued until the mouse fell onto the padded base or until $300 \mathrm{sec}$ had elapsed. Animals received 4 trials per session, with an inter-trial interval of $20 \mathrm{~min}$, and two sessions per day, with an inter-session interval of 1 hour, for 4 days. Latency to fall (s) was the dependent variable. 


\section{Modified Porsolt Forced Swim Test}

This test assesses depressive-like behavioral despair (Cryan et al., 2002). A plastic cylinder (19 $\mathrm{cm}$ tall, $12 \mathrm{~cm}$ wide) was filled up to a height of $10.5 \mathrm{~cm}$ with $25^{\circ} \mathrm{C}$ water. Each animal was tested for 6 minutes. Following each trial, the animal was removed, dried with paper towels, and warmed in cages heated with a heat pad. The water was changed after each trial. Behavior was video recorded and quantified by a blind observer who scored each animal as either engaging in 1) active escape behaviors (swimming, climbing, and diving) or 2) immobility (floating or minimally moving). Behavior of each animal during the first 2 min was not included. Latency (s) to first immobility, total time (s) spent immobile, and number of fecal boli excreted were the dependent variables.

\section{Statistical Analysis}

Statistical analyses were performed with Statview. Differences between groups were analyzed by the unpaired Student's t-test, two-way ANOVA or a repeated measure two-way ANOVA as appropriate for each outcome measure (indicated in the figure legends).

\subsection{Results}

\section{Infarct Area}

Low dose bacterial infection mimic administered 30 minutes prior to a mild (30 minute) tMCAO increased cortical infarct area $(\mathrm{p}<0.05)$ and caudate/putamen infarct area $(\mathrm{p}<0.05)$ (Figure 4.2). We measured rectal temperature at $0,6,12,18,24$, and 48 hours post-stroke to ensure that the increase in infarct volume was not due to an increase in temperature after stroke, and this data was reported in Doll et al. (2015c) Stroke alone caused a modest decline in rectal 
temperature, while LPS + stroke caused a marked hypothermia post-stroke (Doll et al., 2015c). These data indicate that the observed increase in infarct volume caused by the combination of LPS and tMCAO was not due to a febrile response. As such, we then conducted assessments in an additional cohort of mice for the effects of LPS on the behavioral outcomes of stroke.

\section{Modified Neurological Severity Score}

To assess functional neurological damage, the modified neurological severity score (mNSS) adapted from Chen et al. (2005) was administered for 7 days post-surgery. Prior to surgery, the mNSS was administered to assess baseline biases, and animals in all 4 groups scored similarly. Overall there was a Day x Treatment $(\mathrm{p}=0.0067)$ and Day x Surgery interaction $(\mathrm{p}<0.0001)$. Upon visual inspection of the graph, the stroke and LPS effect diminished over time resulting in the effect of surgery or treatment being exacerbated in the acute recovery phase (days $1-3$ ) (Figure 4.3), but in the sub-acute recovery phase (days 4-6), mNSS scores returned to baseline

levels. This rapid recovery after an insult in mice has been noted previously (Bouet et al., 2007; Freret et al., 2009). Thus, we blocked the data by acute recovery phase (days $1-3$ ) and subacute recovery phase (days $4-6$ ) to determine the effect of treatment, surgery, and their interaction on neurological deficits. During the first three days post-surgery, there was an overall Treatment $x$ Surgery interaction $(\mathrm{p}=0.0454)$ indicating that LPS worsens neurological deficits post-stroke. However, during the sub-acute recovery phase the Treatment x Surgery interaction was not observed $(\mathrm{p}=0.7531)$, but the Surgery effect remained $(\mathrm{p}<0.005)$. 


\section{Health and Sickness Behavior Screen}

Prior to tMCAO (time point 0 ), the animals in all four groups had a health screen score of 0 indicating that all animals were healthy animals at the time of treatment administration and/or stroke surgery (Figure 4.4). Overall, there was a Day x Treatment $(\mathrm{p}<0.005)$ and a Day $\mathrm{x}$ Surgery interaction $(\mathrm{p}<0.0001)$. Upon visual inspection of the graph, the surgery and treatment effects were diminished over time, similarly to the mNSS. Thus, we blocked the data by acute recovery phase (days $1-3$ ) and sub-acute recovery phase (days $4-6$ ) to determine the effect of treatment, surgery, and the interaction on sickness behavior. Overall there were no interactions between stroke and LPS during the acute or sub-acute recovery phase. During both block of days there was a significant Surgery effect $(\mathrm{p}<0.0001)$; however, only during the acute phase was there a significant Treatment effect $(\mathrm{p}<0.0005)$. By the sub-acute recovery, the Treatment effect diminished $(\mathrm{p}=0.0842)$, but the Surgery effect remained $(\mathrm{p}<0.0001)$.

\section{Motor Function}

To assess motor function, performance on the accelerating rotarod was assessed $11 \pm 1$ days post-surgery. There was a significant stroke effect resulting in decreased motor function $(\mathrm{p}<$ 0.005; Figure 4.5). This effect of stroke surgery on motor function was not due to decreased locomotor activity because open field indicated no differences between the groups (Table 4.1). To assess forelimb functional asymmetries, the cylinder task was administered. Before surgery (Day 0), there were no differences between groups on rearing (Figure 4.6) nor on left versus right forelimb placement (data not shown). There was an overall Day x Surgery effect $(\mathrm{p}<0.0001)$ post-surgery (Figure 4.6); thus, we further investigated the effect of surgery on each day. There was a significant Surgery effect $(\mathrm{p}<0.0005)$ on rearing on Day 1 , indicating that animals that 
had undergone stroke (regardless of drug treatment) were less active than animals who received sham surgery. By day 3, the surgery effect diminished. This finding of depressed locomotor behavior (as measured by overall number or rears) limits the interpretation of paw placement findings.

\section{Affective Behaviors}

Stroke nor LPS impacted general locomotor ability nor anxiety-like behavior in the open field (Table 4.1). There were no significant group differences in distance moved in any zone during the 6 minute trial (Table 4.1). Similarly, stroke nor LPS impacted anxiety-like behavior on the elevated plus maze. There were no group differences in the number of open arm entries, number of closed arm entries, or the time (s) spent in the open arms (data not shown). Moreover, neither stroke nor LPS impacted depressive-like behavior on the forced swim test. There were no significant treatment effects on total time spent immobile, or latency to first immobility (data not shown).

\subsection{Discussion}

Recent studies have indicated that both Helicobacter pylori (H. pylori) (Wang et al., 2012) and human cytomegalovirus (HCMV) (Huang et al., 2012) infections are associated with increased risk for ischemic stroke. Moreover, having a respiratory tract infection increases the risk for an atherothrombotic ischemic event for up to 3 months (Zurru et al., 2009) and chronic bronchitis increases risk for stroke and transient ischemic attacks (TIA) (Grau et al., 2009). Lastly, Grabska et al. (2011) conducted a retrospective chart review of 2066 ischemic stroke patients to assess the effect of pre-stroke and post-stroke infection on ischemic stroke severity and found that pre-stroke infection increased poor outcomes within the first 30 days but did not have effects 
at 90 days on modified Rankin scores. From a preclinical perspective, LPS has been shown to increase infarct volume when administered prior to MCAO in rodents (McColl et al., 2007; Doll et al., 2015c). However, the long-term effects of stroke during an active infection have not been studied previously. The present study shows for the first time that low dose LPS increases and prolongs sickness behavior and worsens neurological deficits post-stroke. Thus, infection appears to both trigger and worsen severity of stroke.

It is known that a severe stroke results in motor, cognitive, and affective behavioral deficits (Markgraf et al., 1994; Rogers et al., 1997; Gibson and Murphy, 2004; Winter et al., 2005; Bouet et al., 2007). Further, studies have shown that high doses of LPS causes motor, cognitive, and affective deficits (Dunn and Swiergiel, 2005; De Domenico et al., 2010; Hritcu et al., 2011; Painsipp et al., 2011; Tarr et al., 2011; Biesmans et al., 2013). However, until the current study, the long-term functional outcomes of stroke in combination with an active infection had not been methodically assessed in the rodent model. We hypothesized that the LPS administered prior to stroke would exacerbate the motor, cognitive, and affective behavioral deficits post-stroke. Our most robust effects of low dose LPS + mild stroke were on lesion volume, mNSS and sickness. The hypothermic response to LPS + stroke could be an adaptive protective response, because in other studies, we observed that maintenance of core body temperature following LPS + stroke resulted in profound sickness and death of mice (Doll et al., unpublished observations) and that acute post-stroke hypothermia is strongly associated with larger infarct size in mice (Heng et al., unpublished observations). The LPS + stroke effects on mNSS and sickness peaked at one day after stroke, then recovered over the 7-day observation period, indicating that acute behavioral response to LPS + stroke is transient, as has been previously reported for stroke alone in rodents 
and humans (Schaar et al., 2010; Grabska et al., 2011). We also showed that stroke, but not LPS treatment, affects balance and motor coordination during the accelerating rotarod task. As well, we did not observe effects of stroke, LPS, or their combination on open field, elevated plus maze or forced swim. Taken together, we demonstrate for the first time that an infection, present at the time of brain trauma (induced by MCAO) can exacerbate detrimental stroke outcomes, negatively impacting health and significantly prolonging recovery.

Limitations of the present study are several. We did not observe some of the previously reported behavioral deficits that have been found following MCAO or LPS among rodents. This is most likely due to methodological differences between our work and previous studies including 1) the timing of post-manipulation assessments, 2) the severity of the model of brain insult used here, and 3) the low dose of LPS used in our study. Indeed, it is well recognized that there are critical periods for detecting post-stroke deficits (Schaar et al., 2010). As well, many studies assessing functional outcomes following LPS assessed performance within hours of treatment administration. Thus, we may have not seen LPS + stroke effects in some behaviors due to the timing of assessment. Also, severity of the stroke insult may have influenced our ability to detect functional deficits. Our stroke was a 30 minute tMCAO, which is considered a minor insult, and caused a small infarct (Figure 2). The low dose of LPS used here, and thus the severity of the infection induced at the time of stroke also may explain why we did not observe substantial detrimental behavioral changes. Although others have shown that LPS results in motor, cognitive, and affective behavioral deficits, the doses used ranged from $0.3-10 \mathrm{mg} / \mathrm{kg}$ which are 3-100 times higher than the dose used in the present study. Additionally, a few studies injected LPS multiple times before assessing behavior (Hritcu et al., 2011); thus, the 
single, low LPS dose given in this study likely resulted in a minor bacterial infection mimic, and the chosen battery of functional assessments may have not been sensitive enough to detect a mild impairment at the time points at which they were administered. Future studies should assess different doses of LPS and determine the time to assess different behavioral deficits.

Furthermore, an increase prevalence of strokes occurs during flu season. Here, we utilized LPS, a bacterial infection mimic. However, it is not clear whether the exacerbation of post-stroke functional deficits by LPS found here extend to other types of infection. Thus, the assessment of the effects of a viral infection mimic prior to stroke on functional outcome is needed. This study was not designed to assess the mechanism(s) of the interaction between LPS and stroke, but rather to describe the consequences of this interaction on lesion volume, neurological deficits, sickness and behavioral outcomes. There are several potential mechanisms by which LPS could exacerbate stroke outcome. LPS itself is neurotoxic (Lehnardt et al., 2003), and increases levels of several neurotoxic cytokines, including TNF $\alpha$ (McColl et al., 2007). We have shown that TNF $\alpha$ causes neuronal loss by inhibition of mitochondrial oxidative phosphorylation (Doll et al., 2015a). Additionally, LPS compromised the blood-brain barrier (BBB) (Doll et al., 2015c). As such, through either or both mechanisms, treatment with low doses of LPS may sensitize animals to the subsequent effects of a mild stroke and thereby worsen outcomes. Thus, we can conclude from this study that a minor bacterial infection mimic prior to a minor stroke results in increased infarct area, increased sickness behavior and worse neurological deficits resulting in delayed functional recovery. Our observations of the association between infection, stroke, and worse sickness and neurological outcomes argues for the need to 
aggressively treat infection in people with risk factors for stroke and the need to understand the mechanism(s) of this association. 
Table 4.1: Stroke or LPS does not have an effect on locomotor ability in open field. Reported are means \pm standard error of mean for total distance moved $(\mathrm{cm})$, center duration (seconds), inner duration (seconds), and outer duration (seconds) during the open field task for each group.

\begin{tabular}{|l|l|l|l|l|}
\hline Group & $\begin{array}{l}\text { Total } \\
\text { Distance }(\mathrm{cm})\end{array}$ & $\begin{array}{l}\text { Center } \\
\text { Duration }(\mathrm{s})\end{array}$ & $\begin{array}{l}\text { Inner Duration } \\
(\mathrm{s})\end{array}$ & $\begin{array}{l}\text { Outer } \\
\text { Duration }(\mathrm{s})\end{array}$ \\
\hline Vehicle Sham & $2295 \pm 231$ & $5 \pm 2$ & $15 \pm 3$ & $340 \pm 5$ \\
\hline LPS Sham & $2384 \pm 208$ & $5 \pm 1$ & $12 \pm 3$ & $343 \pm 5$ \\
\hline Vehicle Stroke & $2521 \pm 257$ & $5 \pm 1$ & $16 \pm 5$ & $339 \pm 6$ \\
\hline LPS Stroke & $2553 \pm 232$ & $6 \pm 1$ & $16 \pm 2$ & $338 \pm 3$ \\
\hline
\end{tabular}




\section{Figure Legends}

Figure 4.1: Timeline schematic of treatment, surgery and behavioral testing.

Figure 4.2: LPS exacerbates infarct area. Cortical and Caudate/Putamen infarct area. A student's t-test between control and treatment group showed a significant increase in cortical infarct area $(\mathrm{p}=0.0488)$ and caudate/putamen infarct area $(\mathrm{p}=0.0134)$. Depicted are mean \pm SEM for 7 mice/group. $* \mathrm{p}<0.05$

Figure 4.3: Stroke and Stroke + LPS exacerbate neurological deficits. A two way repeated measures ANOVA showed a significant day by treatment $(\mathrm{p}=0.0067)$ and day by surgery effect ( $\mathrm{p}<0.0001$ ). When blocking the data, day $1-3$ (acute phase recovery) and day $4-6$ (sub-acute phase recovery), there was a significant treatment by surgery interaction (0.0454) during the acute phase of recovery. This significant treatment by surgery interaction was lost during the sub-acute phase of recovery. Depicted are mean \pm SEM for 9 to 10 mice/group. When SEM is not shown, it is smaller than the symbol used to depict the mean. $* \mathrm{p}<0.05$

Figure 4.4: Stroke induces sickness behavior. A two way repeated measures ANOVA showed a significant day by treatment $(\mathrm{p}=0.0006)$ and day by surgery effect $(\mathrm{p}<0.0001)$. When blocking the data, day $1-3$ (acute phase recovery) and day $4-6$ (sub-acute phase recovery, there was a significant day by surgery effect $(\mathrm{p}<0.0001)$. Depicted are mean \pm SEM for 9 to 10 mice/group. When SEM is not shown, it is smaller than the symbol used to depict the mean. ** $\mathrm{p}$ $<0.01 ; * * * \mathrm{p}<0.001$

Figure 4.5: Stroke increases motor dysfunction. A two way repeated measures ANOVA showed a significant surgery effect $(\mathrm{p}=0.0011)$. Depicted are mean \pm SEM for 9 to 10 mice/group. ** $\mathrm{p}<0.01$ 
Figure 4.6: Stroke decreases rearing in the cylinder task. A two way repeated measures ANOVA showed a significant day by surgery effect $(\mathrm{p}<0.0001)$. Further investigation of each day indicated a significant effect of surgery on day $1(\mathrm{p}=0.0004)$ but by day 3 there was no effect of surgery or treatment. Depicted are mean \pm SEM for 9 to 10 mice/group. ${ }^{* *} \mathrm{p}<0.01$; $* * * \mathrm{p}<0.001$ 


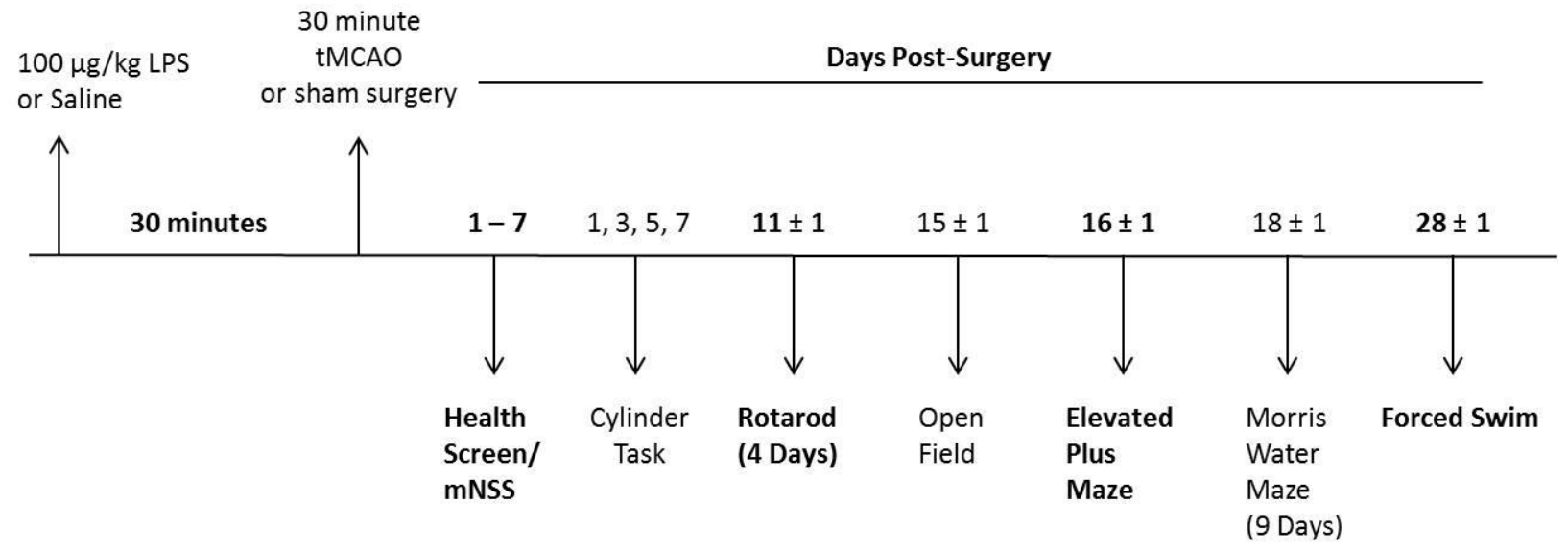

Figure 4.1 

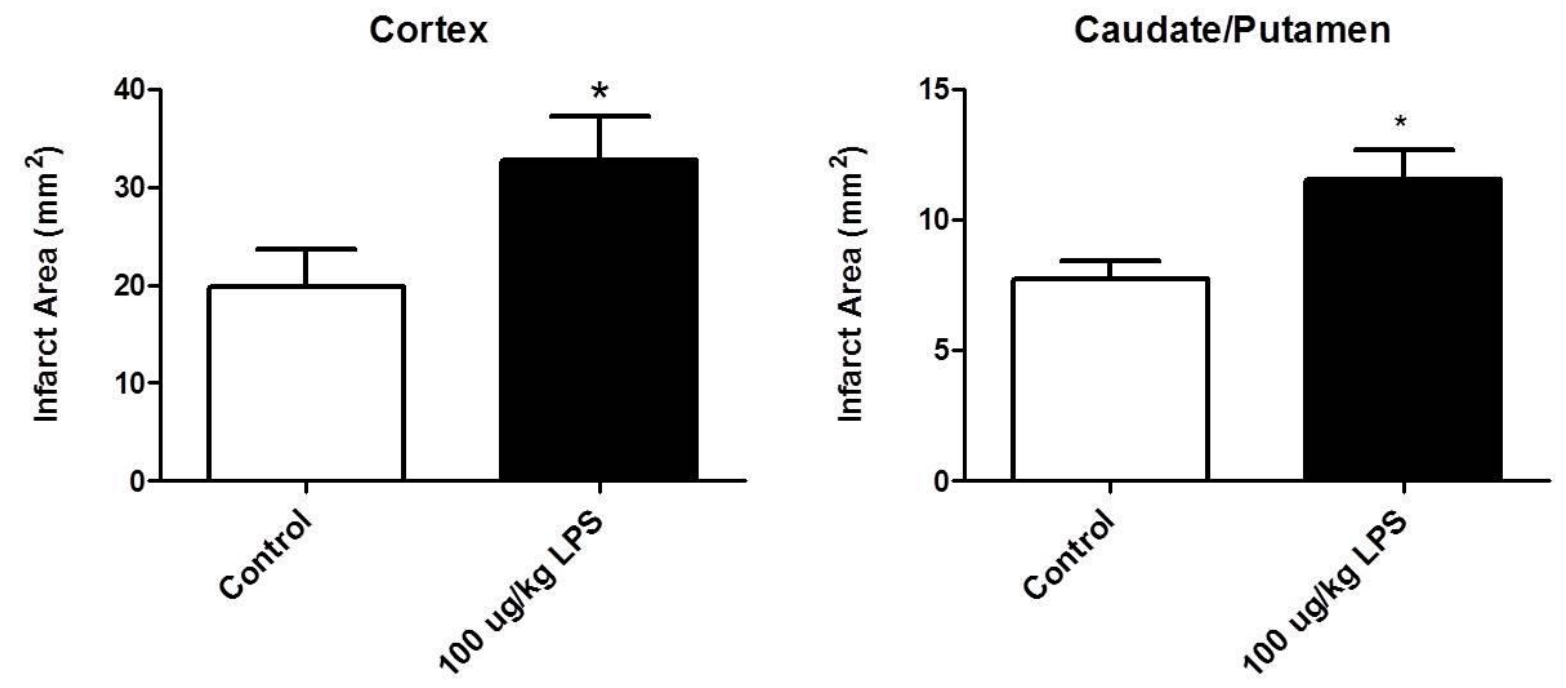

Figure 4.2 


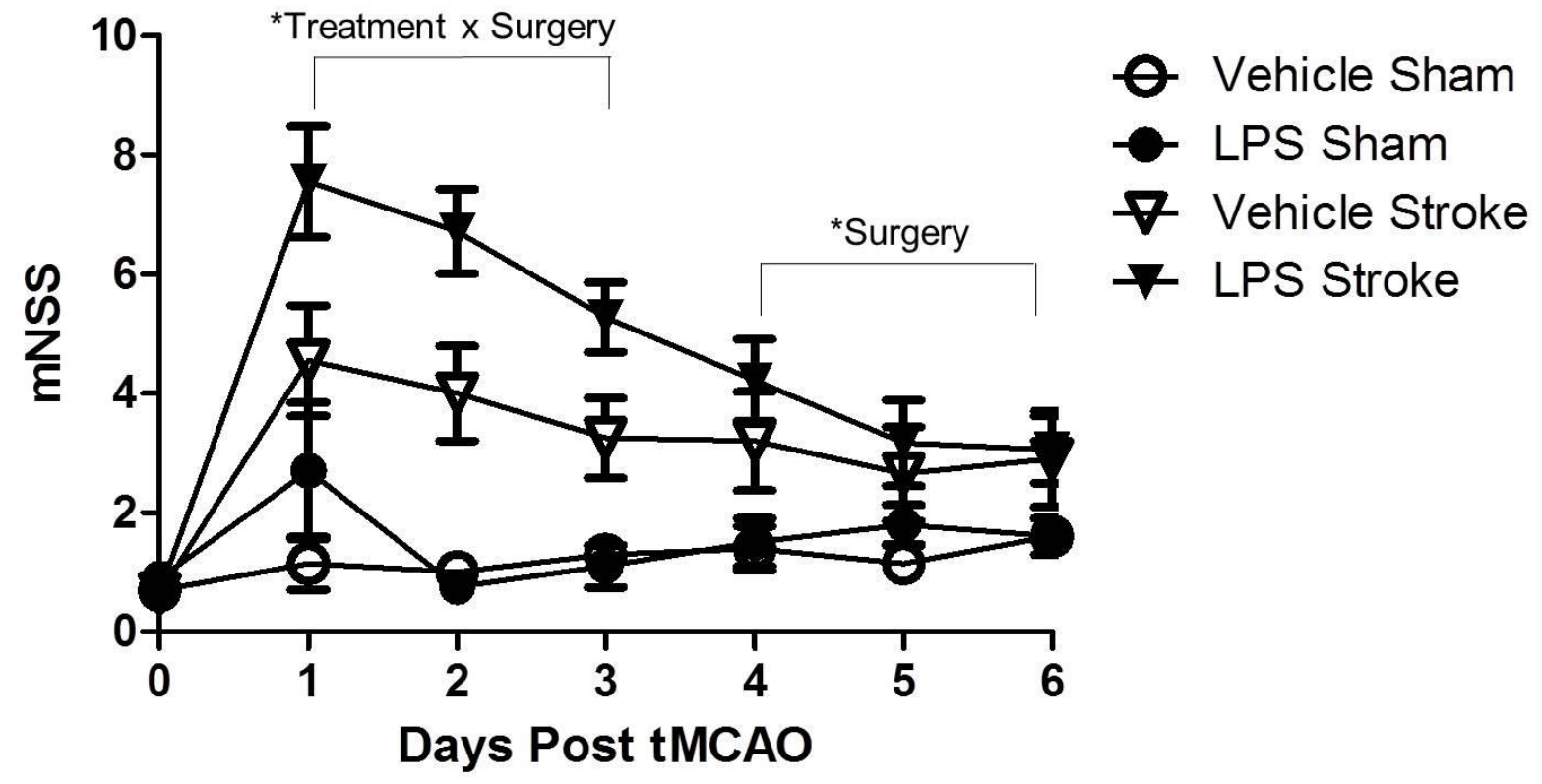

Figure 4.3 


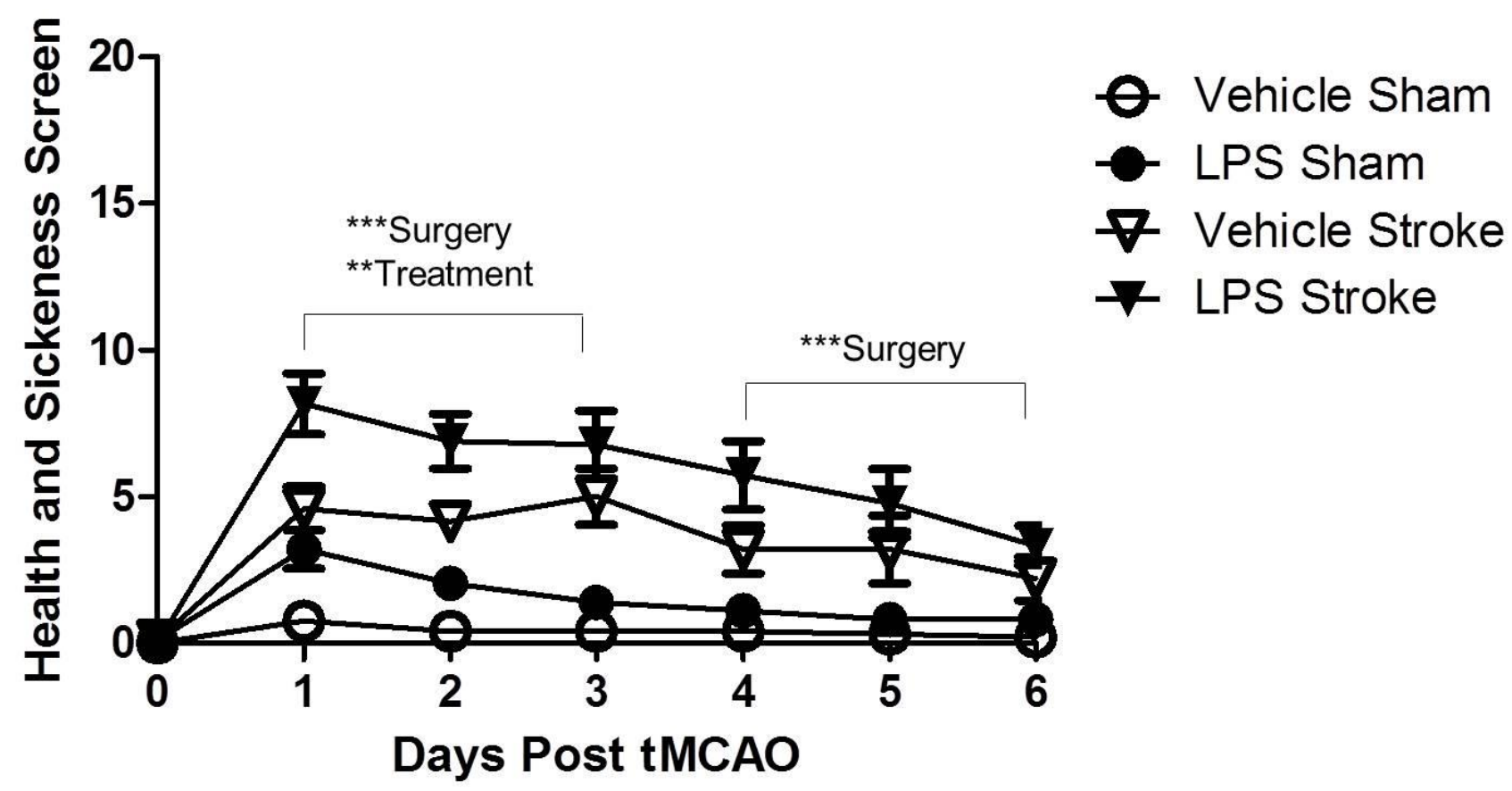

Figure 4.4 


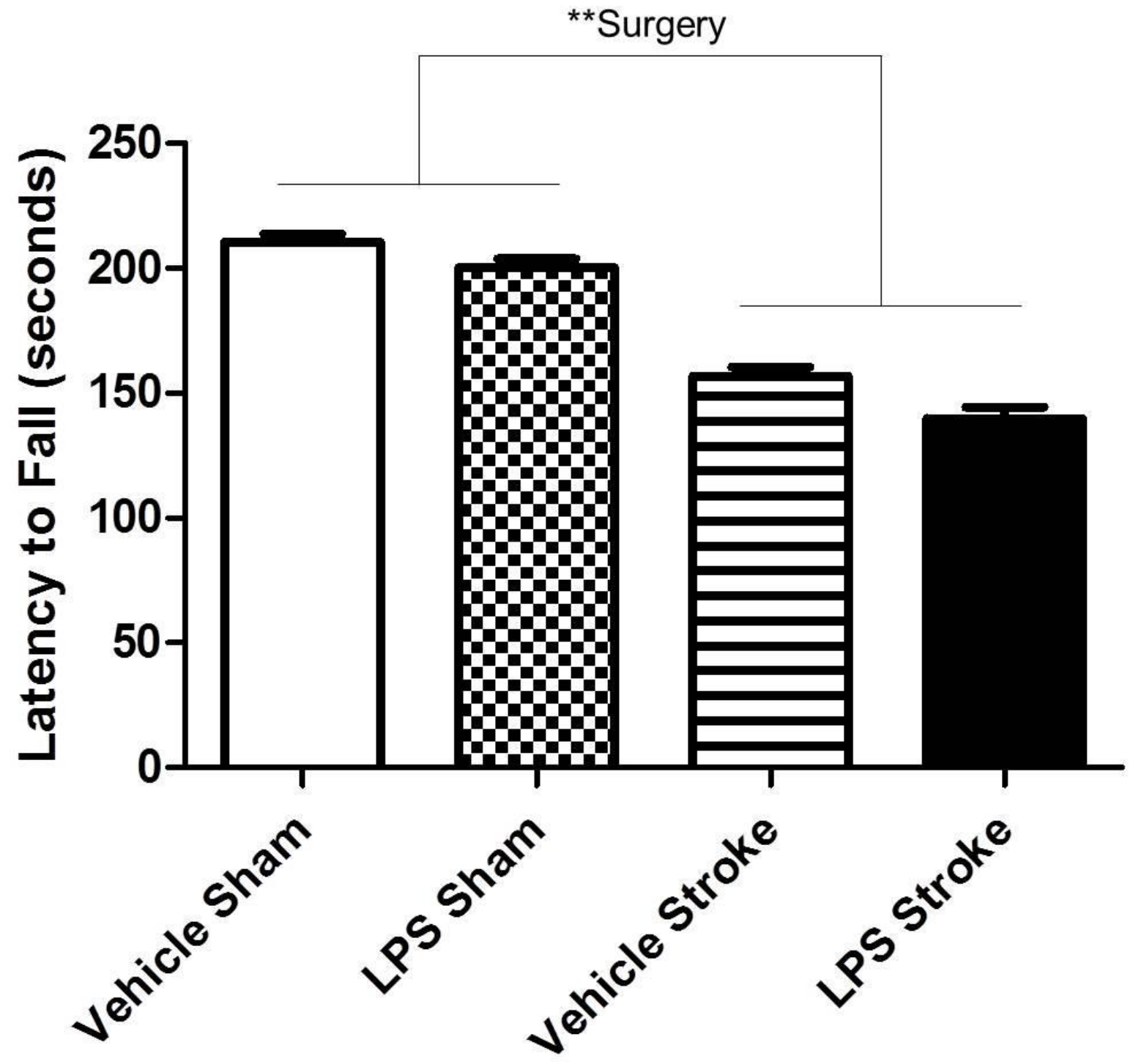

Figure 4.5 


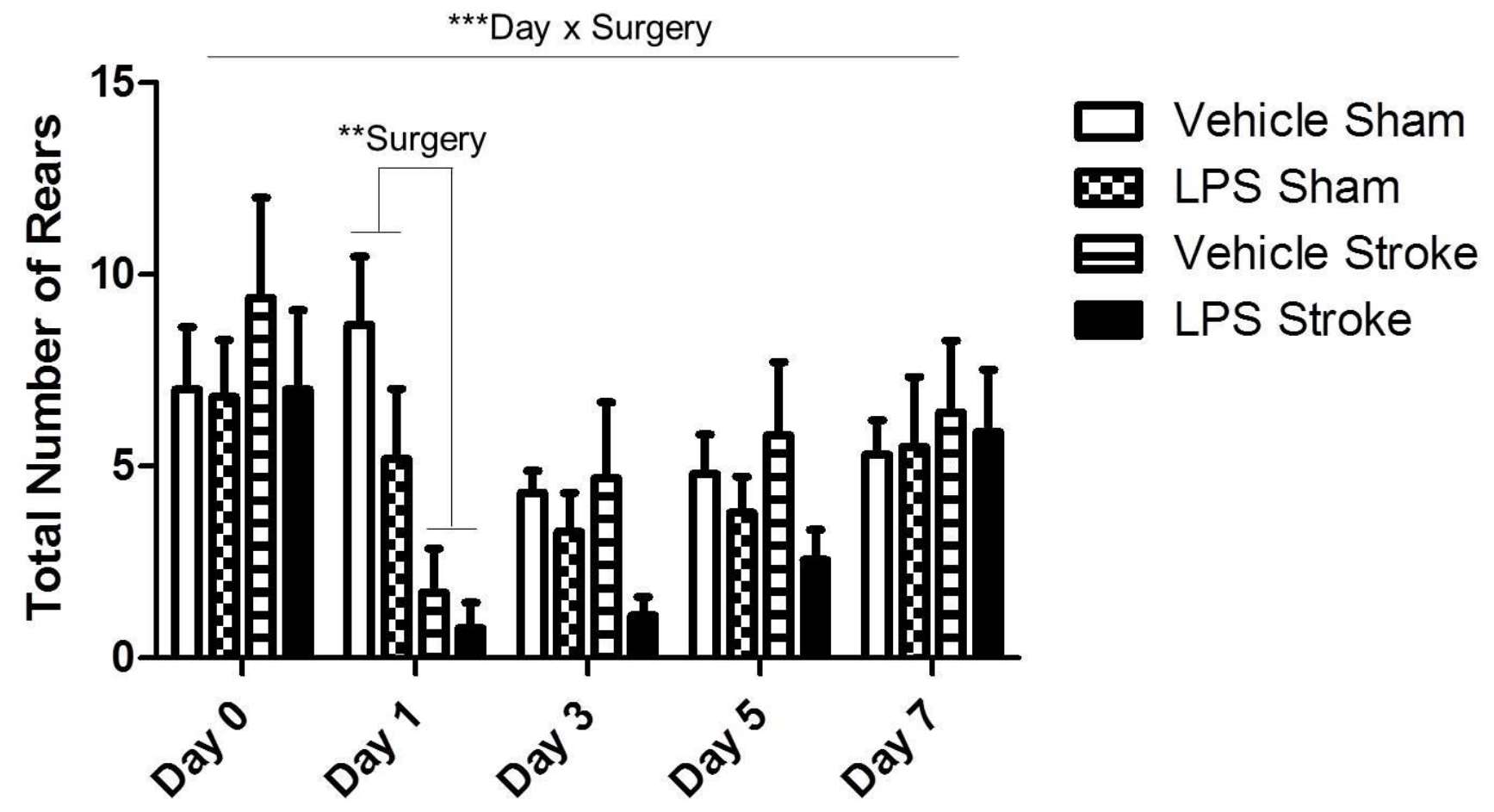

Figure 4.6 
Supplemental Table 4.1: Health and Sickness Behavior Screen.

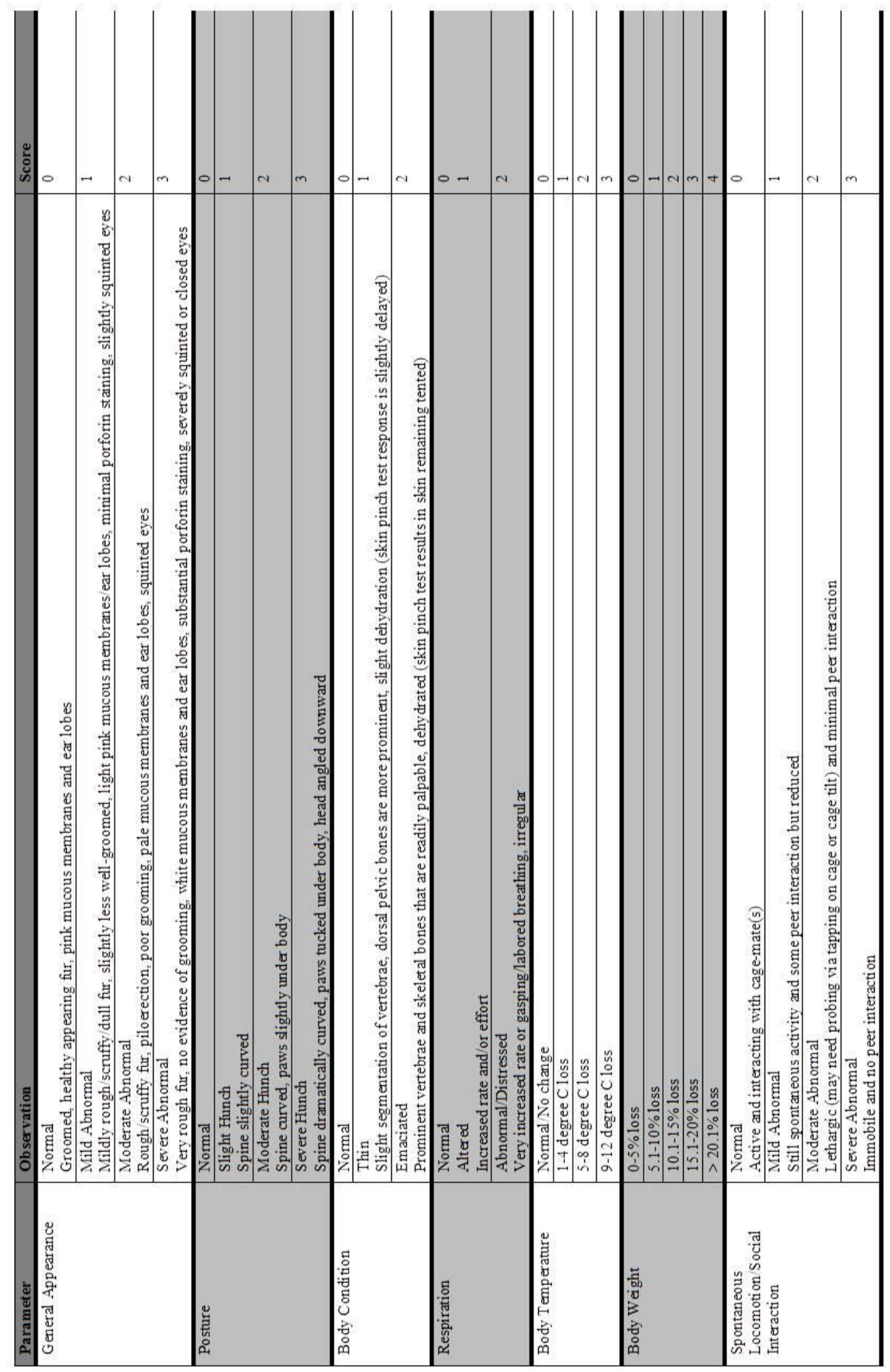




\section{Supplemental Table 4.2: Modified neurological stroke severity score (adapted from Chen} et al., 2005)

Subtest Score

Walking Test 3

Normal

Inability to walk straight

Circling toward paretic side

Falling down toward paretic side

0

1

2

3

Beam Balance Test

6

Balances steadily or traverses beam to clamp $\quad 0$

Grasps sides of beam $(60>\mathrm{x}>50 \mathrm{sec}) \quad 1$

Hugs beam and/or 1 limb slips/falls down $(50>\mathrm{x}>40 \mathrm{sec})$

Hugs beam, 2 limbs slip/fall down and/or spins on beam $(40>x>30 \mathrm{sec}) \quad 3$

Attempts to balance but very unsteady $(30>x>20 \mathrm{sec})$

Attempts to balance but clings to underside of beam $(20>\mathrm{x}>10 \mathrm{sec})$

No attempts to balance and falls $(>10 \mathrm{sec}) \quad 6$

Inverted Test

Forelimb flexion or limb not moving to aid with balance 1

Hindlimb flexion or limb not moving to aid with balance 1

Head moved more than 10 degrees from vertical center or persistent spin 1

$$
\text { Total }=12
$$

***NOTE for Beam Balance: an animal that balances steadily but falls due to traversing or exploring the beam should still score close to zero. Similarly, an animal that balances poorly but stays on for the whole trial should score worse (4-6 range). 


\section{Chapter 5 Discussion}




\section{Discussion}

Understanding the role inflammation plays in the etiology and the pathophysiology of stroke is critical for advancing the field of stroke research. Cytokine production and inflammatory cell infiltration into the brain post-ischemia has been accepted for years; however, the molecular mechanisms underlying this immune response are just now being studied. Despite substantial research and development efforts, there is still only one FDA approved treatment for stroke, tPA. Previous work has explored several mechanisms of neuroprotection through ion channels, excitatory amino acids, and oxygen radicals, but none have culminated in an effective therapeutic effect. Key findings in the field support that inflammatory cells and mediators such as cytokines are important contributing and confounding factors in ischemic brain injury. However, our limited understanding of the multi-faceted and complex events of immune activation following stroke has resulted in many unsuccessful attempts to translate our knowledge of these key inflammatory mediators into therapies for stroke. Inflammatory signaling is involved in the damaging events and the tissue repair stages of the ischemic cascade. Many mediators involved in the ischemic cascade have dual roles; thus, it is important to understand specific inflammatory mediators that act in spatial and temporal relationships to develop effective and novel therapeutic strategies. My dissertation focused on one proinflammatory cytokine, TNF- $\alpha$, which is involved in many co-morbid factors, infection, and post-stroke inflammation. Although TNF- $\alpha$ is clearly not the only cytokine involved, it has been correlated with stroke in numerous clinical studies and assessed extensively in animal models. However, its mechanism of action in neurons at clinically relevant concentrations and exposure times has not been elucidated. 


\section{TNF- $\alpha$ and mitochondrial function}

Our studies show that TNF- $\alpha$ rapidly and profoundly reduces neuronal cell mitochondrial function. It is widely accepted that mitochondria play a role in neuro-inflammation and neurodegenerative CNS disorders such as multiple sclerosis, Alzheimer's disease, and Parkinson's disease (Di Filippo et al., 2010). With an insult like ischemic stroke, activation of microglia occurs that leads to the release of TNF- $\alpha$ along with other pro-inflammatory cytokines (Davalos et al., 2005). Pro-inflammatory cytokines can activate mitochondria-induced apoptosis leading to cell death (Huang et al., 2005). Our findings demonstrate that TNF- $\alpha$ exposure caused neuronal mitochondrial dysfunction as expressed by decreased mitochondrial respiration as early as 1.5 hours of exposure.

Neurons have a high ATP demand (Zhu et al., 2012), and a temporary reduction in ATP production results in a profound decrease in neuronal viability (Simpkins et al., 2010). Fiskum et al. (1999) hypothesized that neuronal death that occurs at the core of an infarct after ischemia results from the decrease in mitochondrial and glycolytic ATP production. TNF- $\alpha$ is known to exacerbate infarct size in pre-clinical models of stroke, and this may be due to the profound and rapid decrease in ATP production, caused by $\mathrm{TNF}-\alpha$ release in the evolving ischemic core. We hypothesized that TNF- $\alpha$ affects mitochondrial function through TNF-R1 because soluble TNF- $\alpha$ signaling is primarily through TNF-R1 (Wajant et al., 2003; Maddahi et al., 2011). Blocking TNF-R1 ameliorated the neurotoxic effects of TNF- $\alpha$ on mitochondrial respiration and cell viability, confirming TNF- $\alpha$ exerts neurotoxic effects through TNF-R1. To further understand the neurotoxic mechanisms of TNF- $\alpha$, we assessed caspase 8 and caspase 3/7 activity because TNF- $\alpha$ can signal through TNF-R1 and activate caspase 8 or caspase $3 / 7$ (Baud and 
Karin, 2001; McCoy and Tansey, 2008). The activation of caspase 8 causes mitochondrialinduced apoptosis through the cleavage of Bcl-2-associated $\mathrm{X}$ protein (BAX) and $\mathrm{BH} 3$ interacting-domain death agonist (BID) (Aggarwal, 2003; McCoy and Tansey, 2008). Caspase 8 activity was increased in a dose-dependent manner after exposure to TNF- $\alpha$ for 1.5 hours; however, caspase $3 / 7$ activity was not affected at this early time. The increase in caspase 8 activity led us to hypothesize that mitochondrial membrane potential should decrease if caspase 8 is initiating mitochondrial-induced apoptosis (Green and Kroemer, 2004). The TMRE mitochondrial membrane potential assay showed a significant dose-dependent decrease in mitochondrial membrane potential after 1.5 hour of TNF- $\alpha$ exposure. Furthermore, with a collapse of mitochondrial membrane potential there should be an increase in cytochrome $c$ release from the mitochondria, due to the cleavage of BAX and BID (Baud and Karin, 2001; Aggarwal, 2003; McCoy and Tansey, 2008). After exposure to $100 \mathrm{pg} / \mathrm{ml}$ of TNF- $\alpha$ for 3 and 24 hours, there was an $11 \%$ and $68 \%$ increase in cytochrome $c$ release, respectively, from mitochondria. Our observation of a $19.7 \%$ decrease in mitochondrial membrane potential and the $30 \%$ decrease in ATP production at 1.5 hours of treatment with $100 \mathrm{pg} / \mathrm{ml} \mathrm{TNF- \alpha} \mathrm{indicates} \mathrm{that} \mathrm{sufficient}$ cytochrome $c$ is released to induce apoptosis (Sas et al., 2007). Once cytochrome $c$ is released, cytochrome $c$ interacts with apoptotic protease-activating factor-1 (Apaf-1), procaspase-9, and dATP forming an apoptosome (Sas et al., 2007). The formation of the apoptosome allows for the activation of caspase- 9 , which activates procaspase- 3 , and results in the induction of apoptosis (Sas et al., 2007).

HT-22 cells and primary mouse cortical neurons both showed a rapid mitochondrial impairment and subsequent cell death in response to low concentrations of TNF- $\alpha$. However, there was a 
notable difference between the two cell types in the timing of the TNF- $\alpha$ induced cell death. Whereas HT-22 cells died within 3 hours of exposure, primary neurons showed cell loss only at 24 hours. The reason for this delayed response of primary neurons is not clear, but is consistent with the comparative resistance of primary neurons to TNF- $\alpha$ in other studies (Reimann-Philipp et al., 2001; Zhao et al., 2001). We suspect, but do not have evidence to support, that primary neurons may have inherent defense mechanisms that resist the neurotoxic effects of a single cytokine, like TNF- $\alpha$ (Tamatani et al., 1999).

Numerous studies reported an association between increased levels of TNF- $\alpha$ mRNA and protein and an exacerbation of ischemic injury (Di Filippo et al., 2010). Administration of TNF- $\alpha-$ blocking antibodies or soluble TNF-R1 prior to inducing stroke reduces infarct volume (Murakami et al., 2005; Di Filippo et al., 2010). Our findings may provide the mechanism of TNF- $\alpha$ exacerbation of stroke damage and shows that TNF- $\alpha$ elicits neurotoxic effects by inducing a rapid mitochondrial dysfunction mediated through TNF-R1 signaling subsequently activating caspase 8 and leading to the release of cytochrome $c$.

Current clinical treatment of ischemic stroke concentrates on dissolving and/or removing the clot, without much focus on the secondary damage that occurs as part of the inflammatory ischemic cascade (Works et al., 2013). Inflammation is known to play a major role in the pathophysiology of stroke and progression of damage post-stroke (Vila et al., 2000a; Iadecola and Anrather, 2011). The identification of a new therapeutic to reduce brain damage due to inflammation post-stroke would be clinically useful. However, TNF- $\alpha$ has both pro-apoptotic and anti-apoptotic activities, and both roles are important physiologically (Aggarwal, 2003). 
When the expression of TNF- $\alpha$ is profound and prolonged, such as after stroke, it has harmful effects (Aggarwal, 2003). Thus, understanding the neurotoxic mechanism of TNF- $\alpha$ is important to further advance its potential as a therapeutic target.

As with all in vitro studies, our studies have some limitations, and we interpreted our results with caution. First, TNF- $\alpha$ was administered at discrete doses (1-1000 pg/ml) for 1.5 to 24 hours. Post- stroke concentrations change over time and are elevated in patients within 6 hours of symptom onset and stay elevated for up to 10 days (Zaremba and Losy, 2001; Intiso et al., 2003). Furthermore, TNF- $\alpha$ mRNA is increased in the ischemic core within 1 hour, and TNF- $\alpha$ immunoreactive protein is seen within 2-6 hours in the brain after middle cerebral artery occlusion, (MCAO) in preclinical models (Liu et al., 1994; Botchkina et al., 1997). We observed mitochondrial impairment with clinically relevant concentrations of TNF- $\alpha$ over a critical time window post-stroke. An additional limitation is the use of cell culture models such as a transformed cell line and a primary mouse neuronal culture. Both cell types are models for neurons, but do not have astrocytes, microglia, or endothelial cells; thus, the results should be taken with caution. In view of the similarity between the two models, we believe we have modeled the effects of TNF- $\alpha$ on nerve cells. Finally, we did not evaluate cleavage of BAX and BID. We assumed the cleavage occurred because caspase 8 cleaves BAX and BID, resulting in cytochrome $c$ release. Our results support that exposure to TNF- $\alpha$ at 1.5 hours results in an increase in caspase 8 activity, and by 3 hours we observed a significant release of cytochrome $c$ from the mitochondria into the cytosol. 
The first part of my dissertation shows, for the first time, a rapid and profound mitochondrial dysfunction in neurons with acute exposure to low doses of TNF- $\alpha$. This neurotoxic effect of TNF- $\alpha$ appears to be mediated by TNF-R1, leading to caspase activation, mitochondrial membrane potential collapse, and mitochondrial release of cytochrome $c$. Collectively these data help to elucidate the neurotoxic mechanisms of $\mathrm{TNF}-\alpha$, and thereby, provides potential targets for cytokine directed therapy for neuroprotection.

\section{Implications}

Post-ischemic inflammation acts through redundant pathways; thus, blocking TNF- $\alpha$ or any one inflammatory mediator will most likely not be effective. Therapeutic strategies blocking multiple inflammatory pathways or neutralizing upstream mediators of the ischemic cascade could be more effective. In experimental models, inhibition of toll like receptors and complement is highly protective; however, the potential downside of blocking multiple inflammatory pathways or neutralizing complement or toll like receptors is worsening of infections after stroke. $30 \%$ of patients develop an infection during the acute phase after stroke, and pneumonia is an independent risk factor for poor outcome and death. Thus, targeting multiple inflammatory pathways or neutralizing upstream mediators may not be effective clinically due to increasing the risk in post-stroke infections.

Indeed the intertwined relationship between the immune system and the CNS is complex and not well understood, but understanding the interaction is essential to harness the full therapeutic potential of the immunology of stroke. Studying the neurotoxic signaling pathways of specific inflammatory mediators that have a spatial and temporal effect such as TNF- $\alpha$, will allow the 
field to develop new therapeutic targets that can target the deleterious effects of the inflammatory mediator specifically. TNF- $\alpha$ has been shown to participated in stroke initiation, progression of brain damage acutely post-stroke, tolerance to ischemia, and repair and recovery after stroke as a mediator and modulator of inflammation. Thus, neutralizing TNF- $\alpha$ with Etanercept or infliximab, FDA approved anti-TNF therapies, will most likely not be clinically effective because 1) low blood-brain barrier permeability of the drugs and 2) its role of control and resolution of inflammation opposing its potent cytotoxicity. Targeting the cytotoxic effect of TNF- $\alpha$ acutely after ischemia will potentially provide neuroprotection through limiting ischemic damage while still allowing TNF- $\alpha$ to be a mediator in the repair phase post-stroke. Since we have shown that TNF- $\alpha$ affects neuronal mitochondrial function, a possible therapeutic intervention could be increasing mitochondrial function or targeting caspase 8 downstream of TNF- $\alpha$ signaling to prevent neuronal cell death and limit brain damage.

Moreover atherosclerosis, diabetes, obesity, and infection cause inflammation that contributes to vascular injury and increases the risk of stroke. TNF- $\alpha$ is an essential cytokine in all of these comorbid factors. Stroke is most commonly caused when an atherosclerotic plaque ruptures, and in particular, TNF- $\alpha$ plays a key role in not only the development of atherosclerotic plaques but their destabilization. When monocytes and macrophages were primed and activated to express high levels of TNF- $\alpha$ when stimulated, rats with no risk factors for stroke were sensitive to induction of brain ischemia by intraventricular administration of LPS (28). Administration of recombinant type I soluble TNF receptor prevented these strokes implicating TNF- $\alpha$ as a necessary causal factor (28). Conventionally, prevention of cerebrovascular disease involve inhibition of platelet aggregation and the coagulation cascade; however, measures to control the 
TNF- $\alpha$ signaling pathway involved in increasing susceptibility stroke could be used as a preventive therapeutic.

In support of using TNF- $\alpha$ antagonists or targeting the TNF signaling pathway as a possible preventive therapeutic for individuals with increased risk for stroke, rheumatic disorders such as rheumatoid arthritis are characterized with chronic and progressive inflammation similar to many co-morbid stroke risk factors. TNF- $\alpha$ is a key cytokine in rheumatic disorders, and various TNF$\alpha$ antagonists are used for treatment. A Danish study with 18,247 rheumatoid arthritis patients, followed for a median of 4.8 years, reported a $30 \%$ higher risk of ischemic stroke compared to those without rheumatoid arthritis (Lindharsen et al., 2012). While traditional stroke risk factors are more prevalent in patients with rheumatoid arthritis, these factors do not fully explain the enhanced cerebrovascular risk in this population. Current research in this field suggests that systemic inflammation is a key player, and rheumatoid arthritis patients taking TNF- $\alpha$ antagonists had a lower risk for ischemic stroke (Naranjo et al., 2008). However, no randomized study has corroborated this trend.

TNF- $\alpha$ participates in every facet of stroke: the etiology, pathophysiology, and recovery and repair phase. The current knowledge of TNF signaling has been studied extensively in nonneuronal cells, and the few studies in neurons use high concentrations and long periods of exposure. Thus, future studies need to focus on the cooperative role for TNF- $\alpha$ in the complex ischemic cascade that regulates neuronal life/death decisions and the response of brain cells to ischemic injury. This improved understanding of TNF mechanism in stroke will improve stroke treatment and prevention. 


\section{Infection and mitochondrial function}

Recent studies have indicated that both Helicobacter pylori (H. pylori) (Wang et al., 2012) and human cytomegalovirus (HCMV) (Huang et al., 2012) infections are associated with increased risk for ischemic stroke. Moreover, having a respiratory tract infection increases the risk for an atherothrombotic ischemic event for up to 3 months (Zurru et al., 2009) and chronic bronchitis increases risk for stroke and transient ischemic attacks (TIA) (Grau et al., 2009). Lastly, Grabska et al. (2011) conducted a retrospective chart review of 2066 ischemic stroke patients to assess the effect of pre-stroke and post-stroke infection on ischemic stroke severity and found that pre-stroke infection increased poor outcomes within the first 30 days but did not have effects at 90 days on modified Rankin scores. Although much is known about the factors that worsen stroke severity in clinical subjects and experimental stroke models, less is understood about their mechanisms.

The blood-brain barrier $(\mathrm{BBB})$ is a highly specialized vascular interface that maintains homeostasis in brain by separating the blood compartment from the central nervous system (CNS). Disruption of cerebrovascular endothelial cells not only allows entry of unwanted solutes into brain, but also disrupts the normal CNS entry route of critical nutrients.(Rubin and Staddon, 1999; Gonzalez-Mariscal et al., 2000) The BBB excludes the majority of bacteria; however, similar to ischemia and reperfusion, inflammation induced by bacteria alters BBB permeability. Thus far, studies have provided evidence that the release of oxidants, proteolytic enzymes and inflammatory cytokines alter BBB permeability. Previous studies found that an LPS challenge results in a larger infarct volume (McColl et al., 2007), impairs survival and disrupts BBB (Denes et al., 2011) in experimental stroke models, through mechanisms that are proposed to 
involve cytokines. As noted, TNF- $\alpha$ is increased during infections along with other co-morbid factors that increase risk for stroke, and because we found that TNF- $\alpha$ rapidly and profoundly decreases mitochondrial function, we hypothesized that the LPS exacerbation of infarct volume is due decreased endothelial mitochondrial function resulting in increased BBB permeability.

Our data are the first to demonstrate that a bacterial infection mimic acts with ischemic challenge to markedly exacerbate stroke damage via a mitochondrial-dependent mechanism. This new finding could also provide an explanation for the association of infections with severity of brain damage and BBB dysfunction from stroke: the induction of hypometabolism in cerebrovascular endothelial cell mitochondria.

Mitochondrial dysfunction is increasingly recognized as an accomplice in vascular diseases and ischemic stroke. More recently, it has been shown that components of the TLR4 signaling pathways, MyD88 and TRAF6, are linked with mitochondria and affect oxidative phosphorylation.(Kim et al., 2007). The expression of MyD88 in mitochondria of endothelial cells supports that mitochondrial function can be affected by LPS. It is notable that we observed no significant effect of LPS is seen on cCVEC basal oxygen consumption and ATP-linked oxygen consumption upon treatment with oligomycin, indicating that LPS does not significantly change basal oxygen demand in mitochondria after 24 hours of treatment. However, the loss of maximal respiration and spare capacity demonstrates that mitochondrial capacity is reduced by LPS and thus the effects exacerbate the mitochondrial impairment by ischemia. Cellular respiration, mitochondrial biogenesis and function, require coordinated expression of proteins encoded by nuclear and mitochondrial genes including mitochondrial complex I-IV. The reduced 
expression of respiratory chain complex I, III and IV proteins in cCVECs by LPS provides further evidence that LPS compromises the endothelial mitochondria function.

The 30 minute tMCAO model mimics a mild ischemic event that results in a small infarct and does not cause visible BBB disruption at 6 hours reperfusion. The bacterial infection mimic challenge prior to tMCAO increased BBB permeability and exacerbated infarct volume. There are inconsistent data for LPS- or cytokine-induced BBB dysfunction (Banks and Erickson, 2010) that may be due to complex mechanisms. McColl et al.(2007) demonstrated an interleukin-1dependent mechanism of stroke exacerbation using the same LPS-tMCAO model. Others have demonstrated that LPS alters transporters or enzymes involved in BBB maintenance (Banks et al., 2008). Our observation that pharmacological inhibition of mitochondria disrupts BBB integrity and increases BBB permeability in vitro and in vivo strengthens the argument that BBB permeability is increased by LPS after stroke through a direct mitochondrial mechanism resulting in a larger infarct volume and worsening neurological deficit.

Increased $\mathrm{BBB}$ permeability, is positively correlated with massive vasogenic and cytotoxic edema post-stroke (Taheri et al., 2009; Yang and Rosenberg, 2011) and elevated neutrophils in the ischemic area that contribute to infarct expansion (Bektas et al., 2010) and poor clinical outcome (Warach and Latour, 2004). Our observation of neutropenia and low NLR may be a consequence of BBB damage that causes rapid migration of neutrophils into damaged tissue. Brain infiltration of neutrophils could also account for secondary lesion growth and increased infarct size in the ischemic brain as seen with LPS treatment. These data may provide translational evidence that neutropenia and low NLR may predict severe stroke. 
We observed that LPS-induced inhibition of mitochondria exacerbates infarct volume and opens BBB in tMCAO mice, but that mitochondria inhibition in cCVECs and pCVECs does not affect cell viability in 24 hours. This suggests that the functional impairment of endothelial mitochondria by LPS may be reversible and an effective intervention could be designed to prevent/restore BBB during strokes. However, our data also indicated that a high dose of LPS $(100 \mu \mathrm{g} / \mathrm{ml})$ induced cell death and reduced cell viability in cCVECs, and extension of LPS exposure for 48 hours induced cell death in both cCVECs and pCVECs. Therefore, the effective mitochondrial directed intervention may be limited by the severity or the length of the infectious exposure to rescue $\mathrm{BBB}$ damage.

Leukocytosis, and cytokine and chemokine storm are features of the acute-phase reaction in systemic infections and in acute stroke. It is becoming increasingly apparent that inflammation can disturb cell energy metabolism. Our recent study found that neuronal mitochondrial function was rapidly and profoundly decreased by $\mathrm{TNF}-\alpha$ resulting in neuronal cell death (Doll et al., 2015a). Another study demonstrated that TNF- $\alpha$ elevated oxygen consumption rate in endothelial cells (Drabarek et al., 2012). The present study did not address the complex LPSinduced effects on mitochondria, however, our novel findings refreshed the traditional idea of infection-inflammation responses and provided a new explanation for the LPS effects on BBB dysfunction: a direct mitochondrial-dependent mechanism.

The application of rotenone on the epidural surface argues that its effect on BBB opening is by compromised mitochondria in vascular endothelial cells. Rotenone exposure is associated with clinical features of parkinsonism in humans (Tanner et al., 2011), and broadly used to induce PD 
like-symptoms in animal models (Chesselet and Richter, 2011) as well. This suggests that the mitochondrial-dependent $\mathrm{BBB}$ opening may not only involve in the progress of stroke but also other neurodegenerative disorders such as PD and AD.

In summary, the second part of my dissertation describes a previously unknown mechanism of infection-induced direct mitochondrial dysfunction in cerebrovascular endothelial cells, which compromises BBB permeability and exacerbates acute stroke outcomes. Moreover, our observations argue that maintenance of mitochondrial function is critical to the integrity of the BBB. The data also suggest a translational significance: maintenance of brain endothelial cell mitochondrial function can improve the acute outcome of stroke and perhaps other neurodegenerative diseases.

\section{Statistical Limitation}

As with all studies there are limitations such as sample size. When a sample size is small, some have cautioned against using the t-test. However, De Winter (2010) provided full analysis of using the Student's t-test with a small sample size. De Winter (2010) reported that the t-test provides Type I error rates close to the 5\% nominal value in most of the cases $(\mathrm{n}=2 \sim 5)$ and that acceptable power $(80 \%)$ is reached only if the effect size is very large. Moreover, William Sealy Gosset developed the t-test for small sample sizes (Lehmann, 2012). Gosset verified his tdistribution on anthropometric data of 3,000 criminals which he divided into 750 samples each having an $n=4$. It was concluded that there are no principle objections to using a t-test with Ns as small as 2. We had an $n=3$ for each group which was replicated 3 times for the experiment 
testing the effects of LPS on brain endothelial cell mitochondrial function, and we had an $\mathrm{n}=3$

for the experiment testing the effects of pharmacological inhibition of mitochondria on $\mathrm{BBB}$ permeability. Both of these studies are in vitro studies which by nature are highly homogeneous resulting in low variances among groups unlike animal and human studies. Thus, a small sample size is acceptable and routinely used in the field.

\section{Implications}

Increased cytokine expression is found centrally and peripherally after ischemic stroke; however, the evidence for central expression of cytokines has predominately come from experimental stroke models and for peripheral expression has come from human studies. Thus, this method of studying cytokines in stroke results in a huge gap in knowledge. Moreover, experimental stroke studies are invasive and could contribute to the cytokine response confounding our understanding of the spatial and temporal response of these cytokines. As noted early, TNF- $\alpha$ has polyphasic roles during ischemia as well as many other of the cytokines. In experimental stroke studies, TNF- $\alpha$ has been shown to induce preconditioning, increase susceptibility, and be neuroprotective. Since chronic over expression or chronic deprivation, via receptor knockout, is not what is endogenously normal, other studies have acutely inhibited TNF- $\alpha$ and showed neuroprotection. Thus, the next phase of research needs to be the assessment of the roles of these complex cytokines during stroke to understand the upstream pathways that regulate the expression of these cytokine and the downstream pathways that amplify inflammation. There is a general consensus in the field that blocking a single cytokine or inflammatory mediator will not be an effective therapeutic intervention. Thus, discovering that TNF- $\alpha$ affects mitochondrial function and that infection prior to ischemia increases BBB permeability through a 
mitochondrial-dependent mechanism suggests that targeting mitochondria could improve stroke outcome.

Mitochondria are the powerhouses of the cells and involved with oxidative phosphorylation, calcium homeostasis, reactive oxygen species (ROS) management, and programmed cell death (Vosler et al., 2009). Disturbing the mitochondrial membrane potential results in caspasedependent and -independent forms of cell death (Taylor et al., 2008; Galluzzi et al., 2009). In caspase-dependent cell death, cytochrome $c$ release activates the caspase cascade causing cleavage of numerous proteins and DNA damage (Taylor et al., 2008). In caspase-independent cell death, calpain - or poly (ADP-ribose) polymerase-1 (PARP1)-mediated apoptosis inducing factor (AIF) is released from the mitochondria and translocates to the nucleus (Galluzzi et al. 2009). Studies inhibiting these downstream mitochondrial death effectors through inhibition of caspases, overexpression of X-lined inhibitor of apoptosis (endogenous caspase inhibitor), HSP70 (binds and sequesters AIF released from the mitochondria), and AIF deletion have shown neuroprotection acutely post-ischemia (24 hours - 1 week) (Galluzzi et al., 2009; Galluzzi et al., 2010). Thus, targeting downstream effectors has only short-term protection which is likely due to the presence of compensatory mechanisms of the mitochondria or to mitochondriaindependent mechanisms like increased oxidative stress.

Since targeting downstream effectors appear to only have short-term protection and the damage done may be too extensive to reverse, targeting direct effectors of mitochondrial membrane disruption may be more advantageous. The Bcl-2 family of pro-death proteins (Bax, Bid, Bad, Bim, and PUMA, among others) are involved with mitochondrial impairment (Youle and 
Strasser, 2008). Bax directly causes mitochondrial membrane disruption through formation of channels in the outer mitochondrial membrane (Youle and Strasser, 2008). Bid and PUMA aid Bax channel formation (Youle and Strasser, 2008). While, Bad and Bim inhibit pro-survival Bcl-2 proteins and Bcl-xL (Youle and Strasser, 2008). Previous work inhibiting pro-death Bcl-2 proteins or increasing pro-survival Bcl-2 proteins has shown neuroprotection (Yin et al., 2006; Steckley et al., 2007). A single gene deletion of Bax, Bid, Bim, or PUMA only provided limited neuroprotection, but a triple knockout mouse with deletions of Bax, Bid, and PUMA showed a prolonged neuroprotection up to 14 days post-ischemia (Vosler et al., 2009). It appears there is a functional redundancy in pro-death Bcl-2 proteins similar to inflammatory signaling pathways; thus, targeting a single pro-death Bcl-2 protein similar to targeting one cytokine is not sufficient. Thus, a potential target that may provide a more robust neuroprotection would be to target natural inhibitors of the pro-death Bcl-2 proteins, pro-survival Bcl-2 proteins or Bcl-xL.

A more attractive target would be to target further upstream to exert more neuroprotection. MAP kinase c-Jun N-terminal kinase (JNK) is consistently activated after cerebral-ischemia (Johnson and Nakamura, 2007). JNK signaling induces programmed cell death in numerous ways; thus, it has high therapeutic potential. JNK activation directly and indirectly regulates pro-death Bcl-2 family proteins to disrupt mitochondrial membrane potential. JNK phosphorylates the scaffolding protein that causes release and translocation of Bax to mitochondria, and JNK phosphorylates Bad and Bim that further activates Bax which inhibits pro-survival Bcl-xL and Bcl-2 proteins (Gao et al., 2005). In addition, JNK signaling alters the transcription of the transcription factor c-Jun which promotes up regulation of Fas, Bim, and TNF- $\alpha$ (Mehta et al., 
2007). Thus, JNK can induce neuronal death through mitochondrial death pathways and by alteration in the genomic response to shift gene expression toward more pre-death mediators. It is important to note that JNK is elevated between 0.5 and 24 hours following tMCAO in rats; thus, clinically targeting JNK would not be limited to a brief time period post-ischemia to exert its neuroprotective roles (Wang et al., 2007b). Inhibiting JNK using intracerebroventricular injection of a pharmacological inhibitor SP600125 or a small peptide inhibitor D-JNKI-1 60 or 30 minutes post ischemia, respectively, provides robust neuroprotection (Borsello et al., 2003; Gao et al., 2005). Moreover, D-JNKI-1 has been administered 6 hours after ischemia and provided long-lasting neuroprotection and neurobehavioral recovery up to 14 days post-ischemia (Esneault et al., 2008). In light of this compelling evidence, targeting JNK signaling is an excellent prospect for clinical neuroprotection.

Lastly, mitochondria are important for cellular homeostasis. Oxidative stress causes extensive mitochondrial fission which results in neuron death. When the mitochondrial fusion protein mitofusion 2 is overexpressed, neuronal death can be prevented (Jahani-Asl et al., 2007). This suggests that tight regulation of fission and fusion is important for neuronal viability. Moreover, hypoxia increases mitochondrial DNA, total mitochondrial number, expression of mitochondrial transcription factors, and the mitochondrial protein HSP60 which suggests mitochondrial biogenesis is an endogenous neuroprotective response (Diaz and Moraes, 2008). After an ischemic insult mitochondria are damaged and autophagy is induced to remove the damaged mitochondria. However, neuronal function is dependent on maintenance of energy levels and cellular homeostasis. Thus, stimulation or enhancement of mitochondrial biogenesis is a novel therapeutic strategy. More research is needed to understand mitochondrial biogenesis in relation 
to stroke, and future research could focus on the role of alternate transcriptional mechanisms, mitochondrial fission and fusion mechanisms, and mitochondrial turnover after ischemia to design new therapeutic targets.

\section{Infection and Post-stroke Outcome}

From a preclinical perspective, LPS has been shown to increase infarct volume when administered prior to MCAO in rodents (McColl et al., 2007; Doll et al., 2015c). However, the long-term effects of stroke during an active infection have not been studied previously. The last part of my dissertation shows for the first time that low dose LPS increases and prolongs sickness behavior and worsens neurological deficits post-stroke. Thus, infection appears to both trigger and worsen severity of stroke.

It is known that a severe stroke results in motor, cognitive, and affective behavioral deficits (Markgraf et al., 1994; Rogers et al., 1997; Gibson and Murphy, 2004; Winter et al., 2005; Bouet et al., 2007). Further, studies have shown that high doses of LPS causes motor, cognitive, and affective deficits (Dunn and Swiergiel, 2005; De Domenico et al., 2010; Hritcu et al., 2011; Painsipp et al., 2011; Tarr et al., 2011; Biesmans et al., 2013). However, until the current study, the long-term functional outcomes of stroke in combination with an active infection had not been methodically assessed in the rodent model. We hypothesized that the LPS administered prior to stroke would exacerbate the motor, cognitive, and affective behavioral deficits post-stroke. Our most robust effects of low dose LPS + mild stroke were on lesion volume, mNSS and sickness. The hypothermic response to LPS + stroke could be an adaptive protective response, because in other studies, we observed that maintenance of core body temperature following LPS + stroke 
resulted in profound sickness and death of mice (Doll et al., unpublished observations) and that acute post-stroke hypothermia is strongly associated with larger infarct size in mice (Heng et al., unpublished observations). The LPS + stroke effects on mNSS and sickness peaked at one day after stroke, then recovered over the 7-day observation period, indicating that acute behavioral response to LPS + stroke is transient, as has been previously reported for stroke alone in rodents and humans (Schaar et al., 2010; Grabska et al., 2011). We also showed that stroke, but not LPS treatment, affects balance and motor coordination during the accelerating rotarod task. As well, we did not observe effects of stroke, LPS, or their combination on open field, elevated plus maze or forced swim. Taken together, we demonstrate for the first time that an infection, present at the time of brain trauma (induced by MCAO) can exacerbate detrimental stroke outcomes, negatively impacting health and significantly prolonging recovery.

Limitations of the present study are several. We did not observe some of the previously reported behavioral deficits that have been found following MCAO or LPS among rodents. This is most likely due to methodological differences between our work and previous studies including 1) the timing of post-manipulation assessments, 2) the severity of the model of brain insult used here, and 3) the low dose of LPS used in our study. Indeed, it is well recognized that there are critical periods for detecting post-stroke deficits (Schaar et al., 2010). As well, many studies assessing functional outcomes following LPS assessed performance within hours of treatment administration. Thus, we may have not seen LPS + stroke effects in some behaviors due to the timing of assessment. Also, severity of the stroke insult may have influenced our ability to detect functional deficits. Our stroke was a 30 minute tMCAO, which is considered a minor insult, and caused a small infarct. The low dose of LPS used here, and thus the severity of the 
infection induced at the time of stroke also may explain why we did not observe substantial detrimental behavioral changes. Although others have shown that LPS results in motor, cognitive, and affective behavioral deficits, the doses used ranged from $0.3-10 \mathrm{mg} / \mathrm{kg}$ which are 3 - 100 times higher than the dose used in the present study. Additionally, a few studies injected LPS multiple times before assessing behavior (Hritcu et al., 2011); thus, the single, low LPS dose given in this study likely resulted in a minor bacterial infection mimic, and the chosen battery of functional assessments may have not been sensitive enough to detect a mild impairment at the time points at which they were administered. Future studies should assess different doses of LPS and determine the time to assess different behavioral deficits. Furthermore, an increase prevalence of strokes occurs during flu season. Here, we utilized LPS, a bacterial infection mimic. However, it is not clear whether the exacerbation of post-stroke functional deficits by LPS found here extend to other types of infection. Thus, the assessment of the effects of a viral infection mimic prior to stroke on functional outcome is needed. This study was not designed to assess the mechanism(s) of the interaction between LPS and stroke, but rather to describe the consequences of this interaction on lesion volume, neurological deficits, sickness and behavioral outcomes. There are several potential mechanisms by which LPS could exacerbate stroke outcome. LPS itself is neurotoxic (Lehnardt et al., 2003), and increases levels of several neurotoxic cytokines, including TNF $\alpha$ (McColl et al., 2007). We have shown that TNF $\alpha$ causes neuronal loss by inhibition of mitochondrial oxidative phosphorylation (Doll et al., 2015a). Additionally, LPS compromised the blood-brain barrier (BBB) (Doll et al., 2015c). As such, through either or both mechanisms, treatment with low doses of LPS may sensitize animals to the subsequent effects of a mild stroke and thereby worsen outcomes. 
Thus, we can conclude from this study that a minor bacterial infection mimic prior to a minor stroke results in increased infarct area, increased sickness behavior and worse neurological deficits resulting in delayed functional recovery. Our observations of the association between infection, stroke, and worse sickness and neurological outcomes argues for the need to aggressively treat infection in people with risk factors for stroke and the need to understand the mechanism(s) of this association.

\section{Statistical Interpretation}

To simultaneously assess the effects of a minor bacterial infection mimic (LPS) and stroke, we designed a two $\mathrm{x}$ two factorial ANOVA with treatment (LPS or saline) and surgery (tMCAO or sham) as our independent variables. We performed a two-way repeated measures ANOVA to assess the effects of stroke and LPS on sickness behavior and neurological deficits. Factorial designs such as the one implemented here are extremely powerful tools with which "to assess the generality of a finding by studying the effects of one independent variable under different conditions, and we evaluate this generality by examining the results of a factorial experiment for an interaction between the factors"... "An interaction is present when the effects of one independent variable on behavior change at the different levels of the second independent variable" (Keppel and Wickens, 194, 201). For sickness behavior, we had a significant Day x Treatment $(\mathrm{p}=0.0006)$ and Day x Surgery $(\mathrm{p}<0.0001)$ interaction. Thus, we blocked our data by days $1-3$ (acute phase post-stroke) and days $4-6$ (subacute phase post-stroke) to assess the simple main effects of treatment or surgery in the absence of a significant interaction between these two independent variables. During the acute phase post-stroke, there was a significant Treatment effect $(p=0.0005)$ and Surgery effect $(p<0.0001)$. However, there was no 
significant Treatment $x$ Surgery interaction $(\mathrm{p}=0.3895)$. This indicates that ignoring whether or not animals had LPS or saline, animals that underwent tMCAO had increased sickness behavior as compared to sham animals. Similarly, animals who received LPS had increased sickness behavior than saline treated animals regardless of surgical status. During the subacute phase, the Surgery effect remained ( $p<0.0001)$, but there was no significant Treatment effect $(p=0.0842)$. We interpreted this data as stroke and LPS affect sickness behavior during the acute phase poststroke, but LPS does not exacerbate the effect of surgery on sickness behavior (no Surgery $\mathrm{x}$ Treatment interaction). For neurological deficit, we had a significant Day x Treatment $(\mathrm{p}=$ $0.0067)$ and Day x Surgery $(\mathrm{p}<0.0001)$ interaction. Thus, we blocked the data similar to sickness behavior to further probe this interaction. During the acute phase (days $1-3$ ), there was an overall Treatment $x$ Surgery interaction $(p=0.0454)$ indicating that the effect of LPS treatment among stroke animals was larger in magnitude than the effect of LPS treatment among sham animals. Thus, the effect of stroke on neurological deficit was exacerbated by LPS treatment present at the time of brain injury. During the subacute phase (days $4-6$ ), the Treatment $x$ Surgery interaction was not observed $(p=0.7531)$, but a main effect of surgery was observed $(\mathrm{p}<0.005)$. By days $4-6$, the neurological deficit observed was due to surgery alone. LPS did not further exacerbate this deficit $(\mathrm{p}=0.7531)$.

\section{Implications}

In at least a third of ischemic stroke cases, the patients lack traditional risk factors and have no apparent cause. These findings and the observation that thirty to forty percent of strokes occurs during or acutely after an infection (Grau et al., 1995b) suggest an association between systemic infection and stroke. Although infection has been implicated in increasing susceptibility to 
stroke, few studies have examined how an infection prior to stroke affects post-stroke outcome. Thus, our findings suggest that an infection prior to stroke increases sickness behavior and worsens neurological deficits resulting in a delayed functional recovery. These findings should warrant change in clinical practice.

Currently, individuals with infections are prescribed antibiotics and advised to get rest and drink fluids. As long as there are no complications, these patients rarely have a follow-up doctor appointments. Since infection is a risk factor for stroke, populations of individuals that have other co-morbid stroke risk factors or are at a higher risk for stroke should be monitored by their family doctors closely during an active infection. Follow-up visits and education about their increased risk for stroke should be implemented into clinical practice to help prevent stroke. Although stroke is the leading cause of disability in the United States and is a huge financial burden, it can be prevented. Thus, clinical practice changes and education are two strategies in preventing a first stroke which is a major goal of the National Stroke Association.

In addition to infection and other co-morbid factors increasing risk for stroke, after having a stroke or a transient ischemic attack (TIA), the risk of stroke within 3 months is between 15 $20 \%$ (Wu et al., 2007). Post-stroke, patients have immune suppression. It is thought that this immune depression is an adaptive response to help prevent autoimmunity against CNS antigens because studies have correlated the amount of infarcted brain tissue with the concentrations of CNS antigens (Jauch et al., 2006). Moreover, a Th1 response against these CNS antigens has been associated with poor 3-month functional outcome (Becker et al., 2011). However, it is unclear whether this stroke-related autoimmunity to brain antigens is a consequence or a causal 
for post-stroke immune suppression. Another mechanism could be over activation of the adrenergic system releasing catecholamines and corticosteroids which is supported by increased serum cortisol and catecholamine levels in patients, and the elevated cortisol levels are an independent predictor of mortality (Anne et al., 2007). Other studies have shown that depletion of noradrenergic terminals in the liver or $\beta$-adrenergic blockade of these receptors alters cytokine expression by hepatic invariant Natural Killer cells (iNKT) resulting in decreased immune suppression, bacterial infections, and decreased mortality in experimental stroke models suggesting adrenergic nerve terminals in peripheral organs mediate the adrenergic post-stroke immune suppression (Wong et al., 2011). Although it is unclear the mechanism by which immune suppression occurs post-stroke, it is known to lead to an increased incidence of infections post-stroke (Famakin, 2014). Recent studies have shown that up to $30 \%$ of stroke patients have pneumonia or urinary tract infection acutely after stroke, and infections are the leading cause of death in stroke patients (Westendorp et al., 2011). This increased risk of infection post-stroke is concerning not only because of its negative impact on outcomes, but the increased risk of infection could lead to 1) increased susceptibility to a second stroke and 2) worsen the neurological outcome of a second stroke.

Currently, clinical trials have treated stroke patients prophylactically with antibiotics to prevent post-stroke infections. The ESPIAS trial had no effect on infection rate or on mortality (Chamorro et al., 2005). However, the PANTHERIS trial found that infection rate was decreased but the mortality rate was not significantly affected. The lack of effect on mortality rate may be due to that the antibiotic treatments are not targeting the endogenous mechanisms proposed above, autoimmunity to CNS antigens or adrenergic suppression. Moreover, 
prophylactically administering antibiotics pre-stroke in high risk patients or post-stroke could result in a worse immune suppression complicating post-stroke outcomes further.

Preconditioning in the stroke field is an attractive preventive method for stroke. Preconditioning is when a sublethal injurious stimulus protects an organ against a subsequent lethal stimulus (Iadecola and Anrather, 2011). Preconditioning with low doses of LPS, transient non-damaging ischemic insult to the brain, and administration of recombinant $\mathrm{T}$ cell receptor ligand (suppresses the infiltration of inflammatory cells) provides neuroprotection and protects the brain from ischemic damage (Subramanian et al., 2009; Bune et al., 2010; Moskowitz et al., 2010; Jensen et al., 2011). Preconditioning studies have shown that a balance of anti- and pro-inflammatory mediators is required for the protection because simply suppressing the production of proinflammatory cytokines or the infiltration of inflammatory cells is not effective (Ohtsuki et al., 1996; Blondeau et al., 2001; Pradillo et al., 2005; Kunz et al., 2007). Thus, prophylactically treating individuals with antibiotics that are at a high risk for stroke is most likely not a beneficial preventive method. However, immune modulation pre- and post-stroke has significant potential. Further understanding of the immunology of stroke is needed and will enable the development of targeted approaches to selectively suppress the deleterious effects of inflammation.

\section{Conclusions}

My dissertation has provided the field of stroke with the knowledge that TNF- $\alpha$ rapidly and profoundly decreases mitochondrial function resulting in neuronal cell death and endothelial cell mitochondrial dysfunction is linked to LPS-induced increased BBB permeability. Moreover, this 
increased BBB permeability by a bacterial infection mimic results in increased ischemic brain damage and delayed functional recovery post-stroke. My research along with others studying mechanistically how the immune system and the ischemic brain interacts is essential to guide therapeutic interventions. Outlined above are various potential therapeutic interventions for ischemic stroke. I believe that more research should focus on the role of mitochondrial biogenesis in neurons because stimulation or enhancement of mitochondria is a novel neuroprotective strategy. Since we show that not only TNF- $\alpha$ exerts its neurotoxicity through mitochondrial dysfunction but BBB permeability is mediated through mitochondrial dysfunction, maintenance of mitochondrial biogenesis or targeting mediators of autophagy to enhance the beneficial effects of mitochondrial preservation may provide neuroprotection at all stages pre and post-ischemia. The current research suggesting the remarkable impact of modulating the immune system has on stroke damage and recovery justifies the field's need to aggressively pursue basic and clinical studies to elucidate the interaction between the immune and nervous system pre- and post-stroke. In the near future, tPA will not be the only treatment for stroke. Strategies to enhance mitochondrial function will be utilized in addition to tPA to improve acute stroke care. 


\section{Appendix}

Table A1: Preliminary assessment of LPS dose and dosing time prior to tMCAO on outcome.

\begin{tabular}{|l|c|l|l|}
\hline LPS Dose & Time prior to tMCAO & $\mathrm{n}$ & \multicolumn{1}{|c|}{ Outcome } \\
\hline $0.01 \mathrm{mg} / \mathrm{kg}$ & 6 hours & 1 & $\begin{array}{l}\text { Decline in body temperature and could not calculate } \\
\text { infarct }\end{array}$ \\
\hline $0.025 \mathrm{mg} / \mathrm{kg}$ & 6 hours & 1 & $\begin{array}{l}\text { Decline in body temperature and could not calculate } \\
\text { infarct }\end{array}$ \\
\hline $0.05 \mathrm{mg} / \mathrm{kg}$ & 6 hours & 1 & $\begin{array}{l}\text { Decline in body temperature and could not calculate } \\
\text { infarct }\end{array}$ \\
\hline $0.2 \mathrm{mg} / \mathrm{kg}$ & 6 hours & 2 & Severe body temperature decline 24 hours post-tMCAO \\
\hline $0.2 \mathrm{mg} / \mathrm{kg}$ & 6 hours (warmed) & 2 & Animals died prior to 24 hour time point \\
\hline $0.5 \mathrm{mg} / \mathrm{kg}$ & 6 hours & 2 & Severe body temperature decline 24 hours post-tMCAO \\
\hline $1 \mathrm{mg} / \mathrm{kg}$ & 6 hours & 2 & Severe body temperature decline 24 hours post-tMCAO \\
\hline $2 \mathrm{mg} / \mathrm{kg}$ & 6 hours & 4 & Severe body temperature decline 24 hours post-tMCAO \\
\hline $0.025 \mathrm{mg} / \mathrm{kg}$ & 30 minutes & 1 & $\begin{array}{l}\text { Did not get body temperature decline, small increase in } \\
\text { infarct compared to control }\end{array}$ \\
\hline $0.1 \mathrm{mg} / \mathrm{kg}$ & 30 minutes & 7 & $\begin{array}{l}\text { Did not get body temperature decline, significant increase } \\
\text { in infarct compared to control }\end{array}$ \\
\hline $0.25 \mathrm{mg} / \mathrm{kg}$ & 30 minutes & 1 & Decline in body temperature $\left(27 .{ }^{\circ} \mathrm{C}\right)$ \\
\hline
\end{tabular}



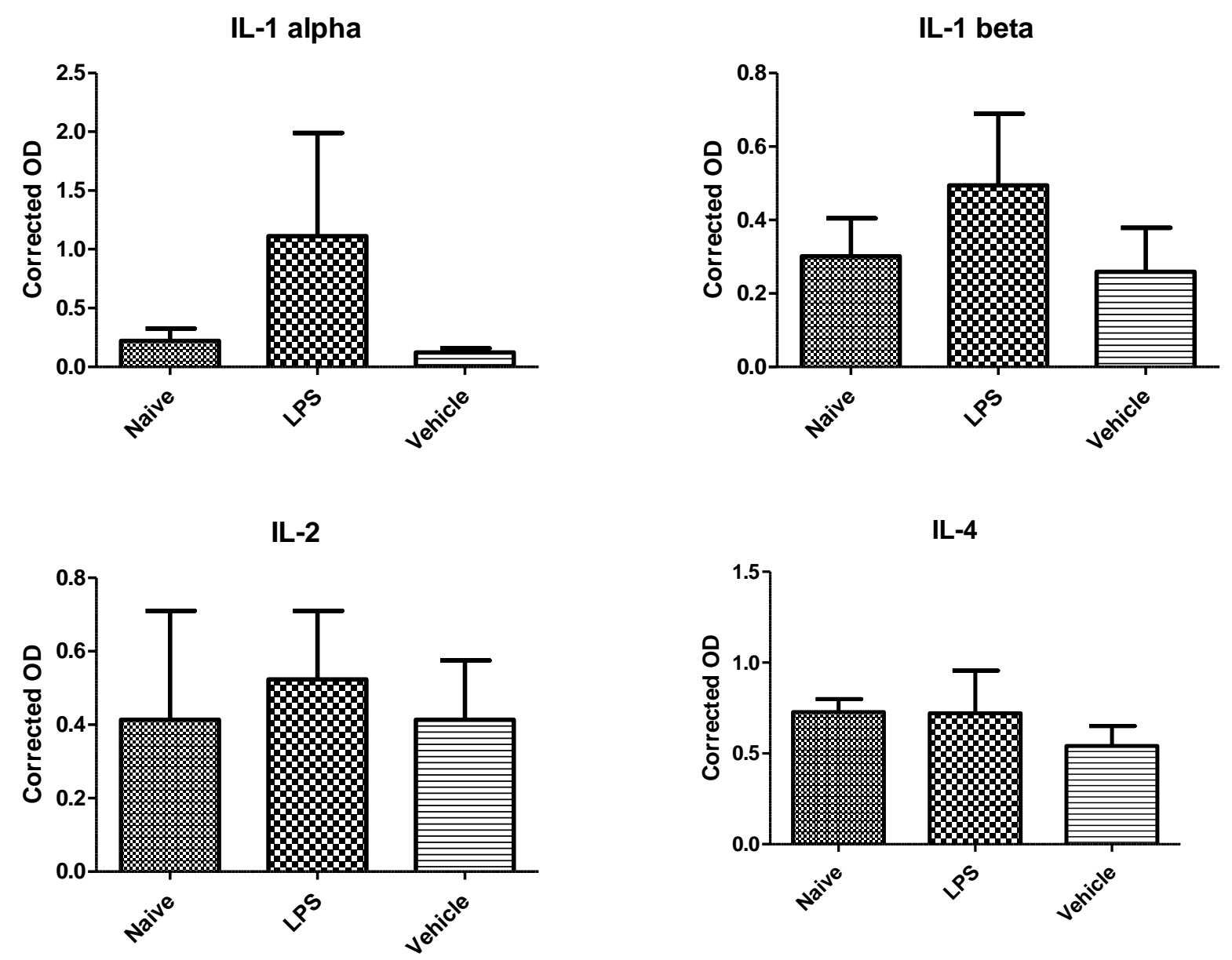

Figure A1: IL-1 alpha, IL-1beta, IL-2, and IL-4 levels 30 minutes after no (naïve), 100 pg/kg LPS, or saline (vehicle) injections prior to any surgery. $n=3 /$ group 

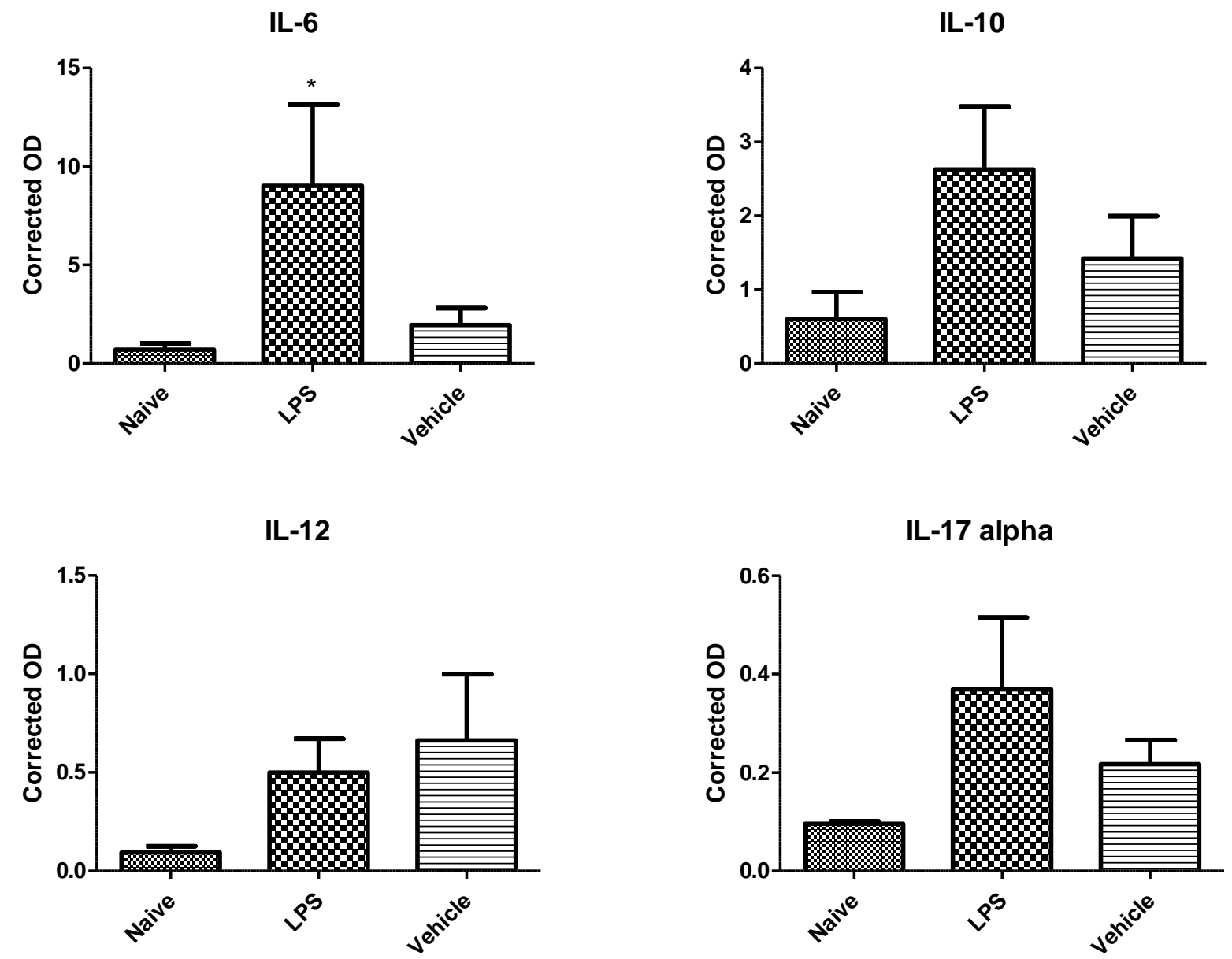

Figure A2: IL-6, IL-10, IL-12, and IL-17 alpha levels 30 minutes after no (naïve), $100 \mu \mathrm{g} / \mathrm{kg}$ LPS, or saline (vehicle) injections prior to any surgery. $n=3$ /group 

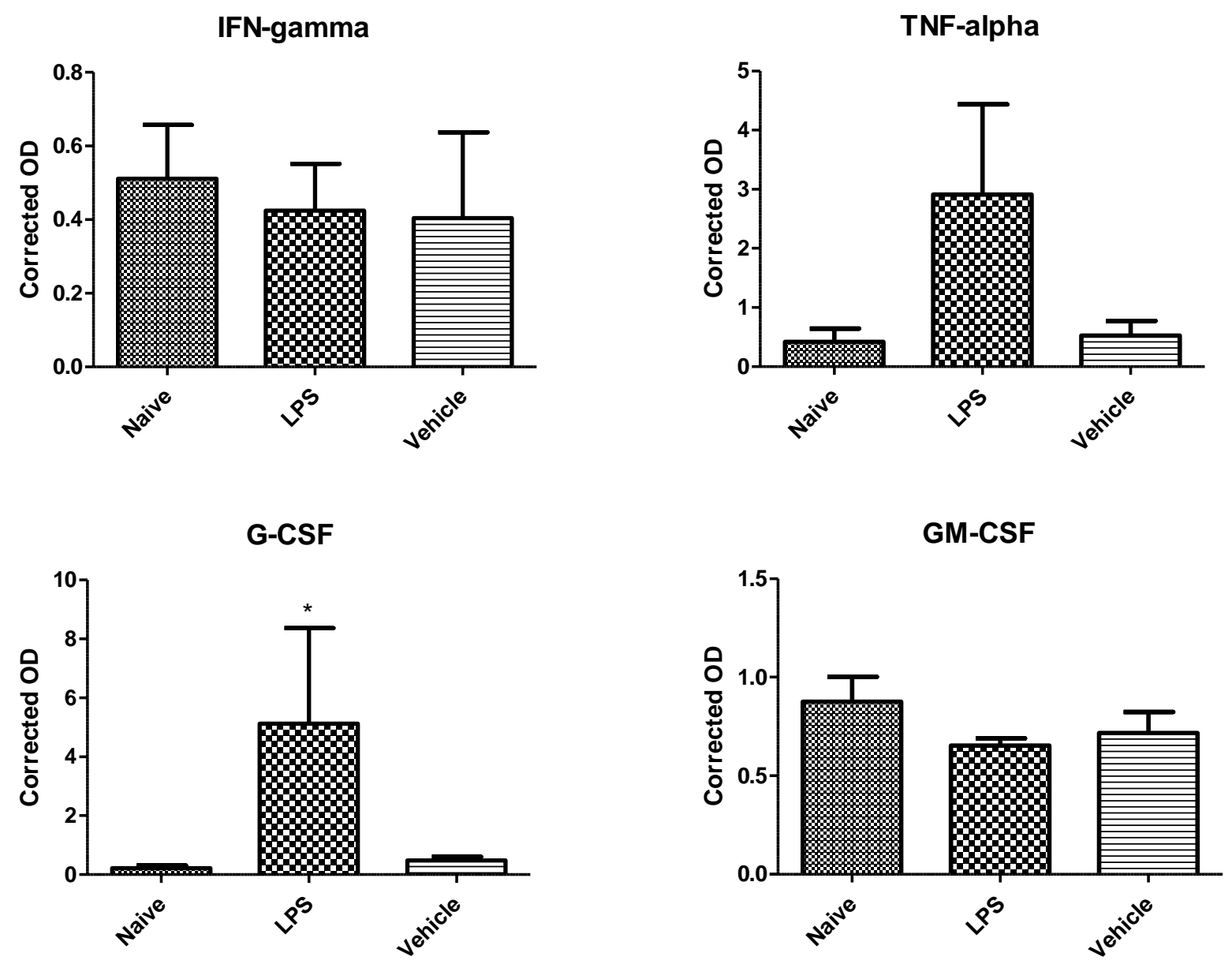

Figure A3: IFN-gamma, TNF-alpha, G-CSF, and GM-CSF levels 30 minutes after no (naïve), $100 \mu \mathrm{g} / \mathrm{kg}$ LPS, or saline (vehicle) injections prior to any surgery. $\mathrm{n}=3$ /group 


\section{References}

Aggarwal BB (2003) Signalling pathways of the TNF superfamily: a double-edged sword. Nature reviews Immunology 3:745-756.

Allan SM, Tyrrell PJ, Rothwell NJ (2005) Interleukin-1 and neuronal injury. Nature reviews Immunology 5:629-640.

American Heart Association (2012) About Stroke. In.

Anne M, Juha K, Timo M, Mikko T, Olli V, Kyosti S, Heikki H, Vilho M (2007) Neurohormonal activation in ischemic stroke: effects of acute phase disturbances on long-term mortality. Curr Neurovasc Res 4:170-175.

Artursson P (1990) Epithelial transport of drugs in cell culture. I: A model for studying the passive diffusion of drugs over intestinal absorptive (Caco-2) cells. Journal of pharmaceutical sciences 79:476-482.

Arvin B, Neville LF, Barone FC, Feuerstein GZ (1996) The role of inflammation and cytokines in brain injury. Neurosci Biobehav Rev 20:445-452.

Astrup J, Siesjo BK, Symon L (1981) Thresholds in cerebral ischemia - the ischemic penumbra. Stroke; a journal of cerebral circulation 12:723-725.

Attwell D, Laughlin SB (2001) An energy budget for signaling in the grey matter of the brain. Journal of cerebral blood flow and metabolism : official journal of the International Society of Cerebral Blood Flow and Metabolism 21:1133-1145.

Azzimondi G, Bassein L, Nonino F, Fiorani L, Vignatelli L, Re G, D'Alessandro R (1995) Fever in acute stroke worsens prognosis. A prospective study. Stroke; a journal of cerebral circulation 26:2040-2043.

Baeuerle PA, Henkel T (1994) Function and activation of NF-kappa B in the immune system. Annual review of immunology 12:141-179.

Banks WA, Erickson MA (2010) The blood-brain barrier and immune function and dysfunction. Neurobiology of disease 37:26-32.

Banks WA, Dohgu S, Lynch JL, Fleegal-DeMotta MA, Erickson MA, Nakaoke R, Vo TQ (2008) Nitric oxide isoenzymes regulate lipopolysaccharide-enhanced insulin transport across the blood-brain barrier. Endocrinology 149:1514-1523.

Bargatze RF, Kurk S, Butcher EC, Jutila MA (1994) Neutrophils roll on adherent neutrophils bound to cytokine-induced endothelial cells via L-selectin on the rolling cells. The Journal of experimental medicine 180:1785-1792.

Barone FC, Arvin B, White RF, Miller A, Webb CL, Willette RN, Lysko PG, Feuerstein GZ (1997) Tumor necrosis factor-alpha. A mediator of focal ischemic brain injury. Stroke; a journal of cerebral circulation 28:1233-1244.

Baud V, Karin M (2001) Signal transduction by tumor necrosis factor and its relatives. Trends in cell biology 11:372-377.

Becker KJ, Kalil AJ, Tanzi P, Zierath DK, Savos AV, Gee JM, Hadwin J, Carter KT, Shibata D, Cain KC (2011) Autoimmune responses to the brain after stroke are associated with worse outcome. Stroke; a journal of cerebral circulation 42:2763-2769.

Bektas H, Wu TC, Kasam M, Harun N, Sitton CW, Grotta JC, Savitz SI (2010) Increased bloodbrain barrier permeability on perfusion CT might predict malignant middle cerebral artery infarction. Stroke; a journal of cerebral circulation 41:2539-2544. 
Beridze M, Sanikidze T, Shakarishvili R, Intskirveli N, Bornstein NM (2011) Selected acute phase CSF factors in ischemic stroke: findings and prognostic value. BMC neurology 11:41.

Bernardes-Silva M, Anthony DC, Issekutz AC, Perry VH (2001) Recruitment of neutrophils across the blood-brain barrier: the role of E- and P-selectins. Journal of cerebral blood flow and metabolism : official journal of the International Society of Cerebral Blood Flow and Metabolism 21:1115-1124.

Berti R, Williams AJ, Moffett JR, Hale SL, Velarde LC, Elliott PJ, Yao C, Dave JR, Tortella FC (2002) Quantitative real-time RT-PCR analysis of inflammatory gene expression associated with ischemia-reperfusion brain injury. Journal of cerebral blood flow and metabolism : official journal of the International Society of Cerebral Blood Flow and Metabolism 22:1068-1079.

Beyaert R, Fiers W (1994) Molecular mechanisms of tumor necrosis factor-induced cytotoxicity. What we do understand and what we do not. FEBS letters 340:9-16.

Biesmans S, Meert TF, Bouwknecht JA, Acton PD, Davoodi N, De Haes P, Kuijlaars J, Langlois X, Matthews LJ, Ver Donck L, Hellings N, Nuydens R (2013) Systemic immune activation leads to neuroinflammation and sickness behavior in mice. Mediators Inflamm 2013:271359.

Blondeau N, Widmann C, Lazdunski M, Heurteaux C (2001) Activation of the nuclear factorkappaB is a key event in brain tolerance. The Journal of neuroscience : the official journal of the Society for Neuroscience 21:4668-4677.

Boring L, Gosling J, Cleary M, Charo IF (1998) Decreased lesion formation in CCR2-/- mice reveals a role for chemokines in the initiation of atherosclerosis. Nature 394:894-897.

Borsello T, Clarke PG, Hirt L, Vercelli A, Repici M, Schorderet DF, Bogousslavsky J, Bonny C (2003) A peptide inhibitor of c-Jun N-terminal kinase protects against excitotoxicity and cerebral ischemia. Nature medicine 9:1180-1186.

Botchkina GI, Meistrell ME, 3rd, Botchkina IL, Tracey KJ (1997) Expression of TNF and TNF receptors (p55 and p75) in the rat brain after focal cerebral ischemia. Molecular medicine (Cambridge, Mass) 3:765-781.

Bouet V, Freret T, Toutain J, Divoux D, Boulouard M, Schumann-Bard P (2007) Sensorimotor and cognitive deficits after transient middle cerebral artery occlusion in the mouse. Exp Neurol 203:555-567.

Brown RC, Morris AP, O'Neil RG (2007a) Tight junction protein expression and barrier properties of immortalized mouse brain microvessel endothelial cells. Brain research 1130:17-30.

Brown WR, Thore CR (2011) Review: cerebral microvascular pathology in ageing and neurodegeneration. Neuropathol Appl Neurobiol 37:56-74.

Brown WR, Moody DM, Thore CR, Challa VR, Anstrom JA (2007b) Vascular dementia in leukoaraiosis may be a consequence of capillary loss not only in the lesions, but in normal-appearing white matter and cortex as well. Journal of the neurological sciences 257:62-66.

Bruunsgaard H, Andersen-Ranberg K, Jeune B, Pedersen AN, Skinhoj P, Pedersen BK (1999) A high plasma concentration of TNF-alpha is associated with dementia in centenarians. $\mathrm{J}$ Gerontol A Biol Sci Med Sci 54:M357-364.

Bune LT, Thaning P, Johansson PI, Bochsen L, Rosenmeier JB (2010) Effects of nucleotides and nucleosides on coagulation. Blood Coagul Fibrinolysis 21:436-441. 
Buttini M, Appel K, Sauter A, Gebicke-Haerter PJ, Boddeke HW (1996) Expression of tumor necrosis factor alpha after focal cerebral ischaemia in the rat. Neuroscience 71:1-16.

Cahill GF, Jr. (2006) Fuel metabolism in starvation. Annu Rev Nutr 26:1-22.

Cardenas A, Moro MA, Leza JC, O'Shea E, Davalos A, Castillo J, Lorenzo P, Lizasoain I (2002) Upregulation of TACE/ADAM17 after ischemic preconditioning is involved in brain tolerance. Journal of cerebral blood flow and metabolism : official journal of the International Society of Cerebral Blood Flow and Metabolism 22:1297-1302.

Castellanos M, Castillo J, Garcia MM, Leira R, Serena J, Chamorro A, Davalos A (2002) Inflammation-mediated damage in progressing lacunar infarctions: a potential therapeutic target. Stroke; a journal of cerebral circulation 33:982-987.

Castelo-Branco C, Soveral I (2014) The immune system and aging: a review. Gynecol Endocrinol 30:16-22.

Chamorro A, Amaro S, Vargas M, Obach V, Cervera A, Torres F, Planas AM (2006) Interleukin 10 , monocytes and increased risk of early infection in ischaemic stroke. Journal of neurology, neurosurgery, and psychiatry 77:1279-1281.

Chamorro A, Horcajada JP, Obach V, Vargas M, Revilla M, Torres F, Cervera A, Planas AM, Mensa J (2005) The Early Systemic Prophylaxis of Infection After Stroke study: a randomized clinical trial. Stroke; a journal of cerebral circulation 36:1495-1500.

Chan FK, Chun HJ, Zheng L, Siegel RM, Bui KL, Lenardo MJ (2000) A domain in TNF receptors that mediates ligand-independent receptor assembly and signaling. Science (New York, NY) 288:2351-2354.

Chao CC, Hu S, Peterson PK (1995) Glia, cytokines, and neurotoxicity. Critical reviews in neurobiology 9:189-205.

Chaturvedi RK, Flint Beal M (2013) Mitochondrial diseases of the brain. Free Radic Biol Med 63:1-29.

Chen J, Zhang C, Jiang H, Li Y, Zhang L, Robin A, Katakowski M, Lu M, Chopp M (2005) Atorvastatin induction of VEGF and BDNF promotes brain plasticity after stroke in mice. J Cereb Blood Flow Metab 25:281-290.

Chen XH, Zhao YP, Xue M, Ji CB, Gao CL, Zhu JG, Qin DN, Kou CZ, Qin XH, Tong ML, Guo XR (2010) TNF-alpha induces mitochondrial dysfunction in 3T3-L1 adipocytes. Molecular and cellular endocrinology 328:63-69.

Cheng B, Christakos S, Mattson MP (1994) Tumor necrosis factors protect neurons against metabolic-excitotoxic insults and promote maintenance of calcium homeostasis. Neuron 12:139-153.

Chesselet MF, Richter F (2011) Modelling of Parkinson's disease in mice. The Lancet Neurology 10:1108-1118.

Chi H, Messas E, Levine RA, Graves DT, Amar S (2004) Interleukin-1 receptor signaling mediates atherosclerosis associated with bacterial exposure and/or a high-fat diet in a murine apolipoprotein E heterozygote model: pharmacotherapeutic implications. Circulation 110:1678-1685.

Chopp M, Li Y, Jiang N, Zhang RL, Prostak J (1996) Antibodies against adhesion molecules reduce apoptosis after transient middle cerebral artery occlusion in rat brain. Journal of cerebral blood flow and metabolism : official journal of the International Society of Cerebral Blood Flow and Metabolism 16:578-584. 
Clark WM, Rinker LG, Lessov NS, Hazel K, Hill JK, Stenzel-Poore M, Eckenstein F (2000) Lack of interleukin-6 expression is not protective against focal central nervous system ischemia. Stroke; a journal of cerebral circulation 31:1715-1720.

Clausen BH, Lambertsen KL, Babcock AA, Holm TH, Dagnaes-Hansen F, Finsen B (2008) Interleukin-1beta and tumor necrosis factor-alpha are expressed by different subsets of microglia and macrophages after ischemic stroke in mice. Journal of neuroinflammation 5:46.

Connolly ES, Jr., Winfree CJ, Springer TA, Naka Y, Liao H, Yan SD, Stern DM, Solomon RA, Gutierrez-Ramos JC, Pinsky DJ (1996) Cerebral protection in homozygous null ICAM-1 mice after middle cerebral artery occlusion. Role of neutrophil adhesion in the pathogenesis of stroke. J Clin Invest 97:209-216.

Coyle JT, Puttfarcken P (1993) Oxidative stress, glutamate, and neurodegenerative disorders. Science (New York, NY) 262:689-695.

Cryan JF, Markou A, Lucki I (2002) Assessing antidepressant activity in rodents: recent developments and future needs. Trends in pharmacological sciences 23:238-245.

Cybulsky MI, Iiyama K, Li H, Zhu S, Chen M, liyama M, Davis V, Gutierrez-Ramos JC, Connelly PW, Milstone DS (2001) A major role for VCAM-1, but not ICAM-1, in early atherosclerosis. J Clin Invest 107:1255-1262.

D'Hooge R, De Deyn PP (2001) Applications of the Morris water maze in the study of learning and memory. Brain research Brain research reviews 36:60-90.

Danton GH, Dietrich WD (2003) Inflammatory mechanisms after ischemia and stroke. Journal of neuropathology and experimental neurology 62:127-136.

Darsalia V, Heldmann U, Lindvall O, Kokaia Z (2005) Stroke-induced neurogenesis in aged brain. Stroke; a journal of cerebral circulation 36:1790-1795.

Davalos D, Grutzendler J, Yang G, Kim JV, Zuo Y, Jung S, Littman DR, Dustin ML, Gan WB (2005) ATP mediates rapid microglial response to local brain injury in vivo. Nature neuroscience 8:752-758.

Davies CA, Loddick SA, Toulmond S, Stroemer RP, Hunt J, Rothwell NJ (1999) The progression and topographic distribution of interleukin-1beta expression after permanent middle cerebral artery occlusion in the rat. Journal of cerebral blood flow and metabolism : official journal of the International Society of Cerebral Blood Flow and Metabolism 19:87-98.

Davies JR, Rudd JH, Fryer TD, Graves MJ, Clark JC, Kirkpatrick PJ, Gillard JH, Warburton EA, Weissberg PL (2005) Identification of culprit lesions after transient ischemic attack by combined $18 \mathrm{~F}$ fluorodeoxyglucose positron-emission tomography and high-resolution magnetic resonance imaging. Stroke; a journal of cerebral circulation 36:2642-2647.

Dawson DA, Martin D, Hallenbeck JM (1996) Inhibition of tumor necrosis factor-alpha reduces focal cerebral ischemic injury in the spontaneously hypertensive rat. Neuroscience letters 218:41-44.

de Bilbao F, Arsenijevic D, Moll T, Garcia-Gabay I, Vallet P, Langhans W, Giannakopoulos P (2009) In vivo over-expression of interleukin-10 increases resistance to focal brain ischemia in mice. Journal of neurochemistry 110:12-22.

De Domenico I, Zhang TY, Koening CL, Branch RW, London N, Lo E, Daynes RA, Kushner JP, Li D, Ward DM, Kaplan J (2010) Hepcidin mediates transcriptional changes that modulate acute cytokine-induced inflammatory responses in mice. J Clin Invest 120:2395-2405. 
DeGraba TJ (1998) The role of inflammation after acute stroke: utility of pursuing anti-adhesion molecule therapy. Neurology 51:S62-68.

del Zoppo GJ (2006) Stroke and neurovascular protection. The New England journal of medicine 354:553-555.

del Zoppo GJ, Sharp FR, Heiss WD, Albers GW (2011) Heterogeneity in the penumbra. Journal of cerebral blood flow and metabolism : official journal of the International Society of Cerebral Blood Flow and Metabolism 31:1836-1851.

Denenberg VH (1969) Open-field bheavior in the rat: what does it mean? Annals of the New York Academy of Sciences 159:852-859.

Denes A, Ferenczi S, Kovacs KJ (2011) Systemic inflammatory challenges compromise survival after experimental stroke via augmenting brain inflammation, blood- brain barrier damage and brain oedema independently of infarct size. Journal of neuroinflammation $8: 164$.

Di Filippo M, Chiasserini D, Tozzi A, Picconi B, Calabresi P (2010) Mitochondria and the link between neuroinflammation and neurodegeneration. Journal of Alzheimer's disease : JAD 20 Suppl 2:S369-379.

Diaz F, Moraes CT (2008) Mitochondrial biogenesis and turnover. Cell Calcium 44:24-35.

DiNapoli VA, Huber JD, Houser K, Li X, Rosen CL (2008) Early disruptions of the blood-brain barrier may contribute to exacerbated neuronal damage and prolonged functional recovery following stroke in aged rats. Neurobiol Aging 29:753-764.

Ding C, He Q, Li PA (2005) Diabetes increases expression of ICAM after a brief period of cerebral ischemia. J Neuroimmunol 161:61-67.

Dirnagl U, Iadecola C, Moskowitz MA (1999) Pathobiology of ischaemic stroke: an integrated view. Trends Neurosci 22:391-397.

Doll DN, Rellick SL, Barr TL, Ren X, Simpkins JW (2015a) Rapid mitochondrial dysfunction mediates TNF-alpha-induced neurotoxicity. Journal of neurochemistry 132:443-451.

Doll DN, Hu H, Sun J, Lewis SE, Simpkins JW, Ren X (2015b) Mitochondrial crisis in cerebrovascular endothelial cells opens the blood-brain barrier. Stroke; a journal of cerebral circulation 46:1681-1689.

Doll DN, Hu H, Sun J, Lewis SE, Simpkins JW, Ren X (2015c) Mitochondrial Crisis in Cerebrovascular Endothelial Cells Opens the Blood-Brain Barrier. Stroke.

Dong ZM, Brown AA, Wagner DD (2000) Prominent role of P-selectin in the development of advanced atherosclerosis in ApoE-deficient mice. Circulation 101:2290-2295.

Drabarek B, Dymkowska D, Szczepanowska J, Zablocki K (2012) TNFalpha affects energy metabolism and stimulates biogenesis of mitochondria in EA.hy926 endothelial cells. The international journal of biochemistry \& cell biology 44:1390-1397.

Dunn AJ, Swiergiel AH (2005) Effects of interleukin-1 and endotoxin in the forced swim and tail suspension tests in mice. Pharmacol Biochem Behav 81:688-693.

Dziewulska D, Mossakowski MJ (2003) Cellular expression of tumor necrosis factor a and its receptors in human ischemic stroke. Clinical neuropathology 22:35-40.

Ehrensperger E, Minuk J, Durcan L, Mackey A, Wolfson C, Fontaine AM, Cote R (2005) Predictive value of soluble intercellular adhesion molecule-1 for risk of ischemic events in individuals with cerebrovascular disease. Cerebrovasc Dis 20:456-462.

Emsley HC, Hopkins SJ (2008) Acute ischaemic stroke and infection: recent and emerging concepts. Lancet Neurol 7:341-353. 
Emsley HC, Smith CJ, Gavin CM, Georgiou RF, Vail A, Barberan EM, Illingworth K, Scarth S, Wickramasinghe V, Hoadley ME, Rothwell NJ, Tyrrell PJ, Hopkins SJ (2007) Clinical outcome following acute ischaemic stroke relates to both activation and autoregulatory inhibition of cytokine production. BMC neurology 7:5.

Engler-Chiurazzi EB, Talboom JS, Braden BB, Tsang CW, Mennenga S, Andrews M, Demers LM, Bimonte-Nelson HA (2012) Continuous estrone treatment impairs spatial memory and does not impact number of basal forebrain cholinergic neurons in the surgically menopausal middle-aged rat. Hormones and behavior 62:1-9.

Erta M, Quintana A, Hidalgo J (2012) Interleukin-6, a major cytokine in the central nervous system. International journal of biological sciences 8:1254-1266.

Esneault E, Castagne V, Moser P, Bonny C, Bernaudin M (2008) D-JNKi, a peptide inhibitor of c-Jun N-terminal kinase, promotes functional recovery after transient focal cerebral ischemia in rats. Neuroscience 152:308-320.

Famakin BM (2014) The Immune Response to Acute Focal Cerebral Ischemia and Associated Post-stroke Immunodepression: A Focused Review. Aging Dis 5:307-326.

Fassbender K, Schmidt R, Mossner R, Daffertshofer M, Hennerici M (1994) Pattern of activation of the hypothalamic-pituitary-adrenal axis in acute stroke. Relation to acute confusional state, extent of brain damage, and clinical outcome. Stroke; a journal of cerebral circulation 25:1105-1108.

Feigin VL et al. (2014) Global and regional burden of stroke during 1990-2010: findings from the Global Burden of Disease Study 2010. Lancet 383:245-254.

Ferrarese C, Mascarucci P, Zoia C, Cavarretta R, Frigo M, Begni B, Sarinella F, Frattola L, De Simoni MG (1999) Increased cytokine release from peripheral blood cells after acute stroke. Journal of cerebral blood flow and metabolism : official journal of the International Society of Cerebral Blood Flow and Metabolism 19:1004-1009.

Fillatreau S, Sweenie CH, McGeachy MJ, Gray D, Anderton SM (2002) B cells regulate autoimmunity by provision of IL-10. Nature immunology 3:944-950.

Finbloom DS, Winestock KD (1995) IL-10 induces the tyrosine phosphorylation of tyk2 and Jak1 and the differential assembly of STAT1 alpha and STAT3 complexes in human T cells and monocytes. Journal of immunology (Baltimore, Md : 1950) 155:1079-1090.

Fiskum G, Murphy AN, Beal MF (1999) Mitochondria in neurodegeneration: acute ischemia and chronic neurodegenerative diseases. Journal of cerebral blood flow and metabolism : official journal of the International Society of Cerebral Blood Flow and Metabolism 19:351-369.

Fonarow GC, Smith EE, Saver JL, Reeves MJ, Hernandez AF, Peterson ED, Sacco RL, Schwamm LH (2011) Improving door-to-needle times in acute ischemic stroke: the design and rationale for the American Heart Association/American Stroke Association's Target: Stroke initiative. Stroke; a journal of cerebral circulation 42:2983-2989.

Freret T, Bouet V, Leconte C, Roussel S, Chazalviel L, Divoux D, Schumann-Bard P, Boulouard M (2009) Behavioral deficits after distal focal cerebral ischemia in mice: Usefulness of adhesive removal test. Behav Neurosci 123:224-230.

Galluzzi L, Morselli E, Kepp O, Kroemer G (2009) Targeting post-mitochondrial effectors of apoptosis for neuroprotection. Biochimica et biophysica acta 1787:402-413.

Galluzzi L, Morselli E, Vitale I, Kepp O, Senovilla L, Criollo A, Servant N, Paccard C, Hupe P, Robert T, Ripoche H, Lazar V, Harel-Bellan A, Dessen P, Barillot E, Kroemer G (2010) 
miR-181a and miR-630 regulate cisplatin-induced cancer cell death. Cancer Res 70:1793-1803.

Gao Y, Signore AP, Yin W, Cao G, Yin XM, Sun F, Luo Y, Graham SH, Chen J (2005) Neuroprotection against focal ischemic brain injury by inhibition of c-Jun N-terminal kinase and attenuation of the mitochondrial apoptosis-signaling pathway. Journal of cerebral blood flow and metabolism : official journal of the International Society of Cerebral Blood Flow and Metabolism 25:694-712.

Garcia JH, Liu KF, Relton JK (1995) Interleukin-1 receptor antagonist decreases the number of necrotic neurons in rats with middle cerebral artery occlusion. The American journal of pathology 147:1477-1486.

Gay NJ, Symmons MF, Gangloff M, Bryant CE (2014) Assembly and localization of Toll-like receptor signalling complexes. Nature reviews Immunology 14:546-558.

Gee JM, Kalil A, Shea C, Becker KJ (2007) Lymphocytes: potential mediators of postischemic injury and neuroprotection. Stroke; a journal of cerebral circulation 38:783-788.

Gibson CL, Murphy SP (2004) Progesterone enhances functional recovery after middle cerebral artery occlusion in male mice. J Cereb Blood Flow Metab 24:805-813.

Gonzalez-Mariscal L, Betanzos A, Avila-Flores A (2000) MAGUK proteins: structure and role in the tight junction. Seminars in cell \& developmental biology 11:315-324.

Gourin CG, Shackford SR (1997) Production of tumor necrosis factor-alpha and interleukin1beta by human cerebral microvascular endothelium after percussive trauma. The Journal of trauma 42:1101-1107.

Grabska K, Gromadzka G, Czlonkowska A (2011) Infections and ischemic stroke outcome. Neurol Res Int 2011:691348.

Grau AJ, Preusch MR, Palm F, Lichy C, Becher H, Buggle F (2009) Association of symptoms of chronic bronchitis and frequent flu-like illnesses with stroke. Stroke 40:3206-3210.

Grau AJ, Buggle F, Steichen-Wiehn C, Heindl S, Banerjee T, Seitz R, Winter R, Forsting M, Werle E, Bode C, et al. (1995a) Clinical and biochemical analysis in infection-associated stroke. Stroke; a journal of cerebral circulation 26:1520-1526.

Grau AJ, Buggle F, Heindl S, Steichen-Wiehn C, Banerjee T, Maiwald M, Rohlfs M, Suhr H, Fiehn W, Becher H, et al. (1995b) Recent infection as a risk factor for cerebrovascular ischemia. Stroke; a journal of cerebral circulation 26:373-379.

Grau AJ, Buggle F, Becher H, Zimmermann E, Spiel M, Fent T, Maiwald M, Werle E, Zorn M, Hengel H, Hacke W (1998) Recent bacterial and viral infection is a risk factor for cerebrovascular ischemia: clinical and biochemical studies. Neurology 50:196-203.

Green DR, Kroemer G (2004) The pathophysiology of mitochondrial cell death. Science (New York, NY) 305:626-629.

Gregersen R, Lambertsen K, Finsen B (2000) Microglia and macrophages are the major source of tumor necrosis factor in permanent middle cerebral artery occlusion in mice. Journal of cerebral blood flow and metabolism : official journal of the International Society of Cerebral Blood Flow and Metabolism 20:53-65.

Griffin WS (2006) Inflammation and neurodegenerative diseases. Am J Clin Nutr 83:470S-474S.

Grilli M, Barbieri I, Basudev H, Brusa R, Casati C, Lozza G, Ongini E (2000) Interleukin-10 modulates neuronal threshold of vulnerability to ischaemic damage. The European journal of neuroscience 12:2265-2272. 
Hakkoum D, Stoppini L, Muller D (2007) Interleukin-6 promotes sprouting and functional recovery in lesioned organotypic hippocampal slice cultures. Journal of neurochemistry 100:747-757.

Hallenbeck JM (1996) Significance of the inflammatory response in brain ischemia. Acta Neurochir Suppl 66:27-31.

Hallenbeck JM (2002) The many faces of tumor necrosis factor in stroke. Nature medicine 8:1363-1368.

Hankey GJ (2006) Potential new risk factors for ischemic stroke: what is their potential? Stroke; a journal of cerebral circulation 37:2181-2188.

Hansson GK, Libby P (2006) The immune response in atherosclerosis: a double-edged sword. Nature reviews Immunology 6:508-519.

Hara H, Fink K, Endres M, Friedlander RM, Gagliardini V, Yuan J, Moskowitz MA (1997a) Attenuation of transient focal cerebral ischemic injury in transgenic mice expressing a mutant ICE inhibitory protein. Journal of cerebral blood flow and metabolism : official journal of the International Society of Cerebral Blood Flow and Metabolism 17:370-375.

Hara H, Friedlander RM, Gagliardini V, Ayata C, Fink K, Huang Z, Shimizu-Sasamata M, Yuan J, Moskowitz MA (1997b) Inhibition of interleukin 1beta converting enzyme family proteases reduces ischemic and excitotoxic neuronal damage. Proceedings of the National Academy of Sciences of the United States of America 94:2007-2012.

Harhausen D, Khojasteh U, Stahel PF, Morgan BP, Nietfeld W, Dirnagl U, Trendelenburg G (2010) Membrane attack complex inhibitor CD59a protects against focal cerebral ischemia in mice. Journal of neuroinflammation 7:15.

Harman D (1972) The biologic clock: the mitochondria? J Am Geriatr Soc 20:145-147.

Harris JJ, Attwell D (2012) The energetics of CNS white matter. The Journal of neuroscience : the official journal of the Society for Neuroscience 32:356-371.

Herrmann O, Tarabin V, Suzuki S, Attigah N, Coserea I, Schneider A, Vogel J, Prinz S, Schwab S, Monyer H, Brombacher F, Schwaninger M (2003) Regulation of body temperature and neuroprotection by endogenous interleukin- 6 in cerebral ischemia. Journal of cerebral blood flow and metabolism : official journal of the International Society of Cerebral Blood Flow and Metabolism 23:406-415.

Hill JK, Gunion-Rinker L, Kulhanek D, Lessov N, Kim S, Clark WM, Dixon MP, Nishi R, Stenzel-Poore MP, Eckenstein FP (1999) Temporal modulation of cytokine expression following focal cerebral ischemia in mice. Brain research 820:45-54.

Hogg S (1996) A review of the validity and variability of the elevated plus-maze as an animal model of anxiety. Pharmacology, biochemistry, and behavior 54:21-30.

Howarth C, Peppiatt-Wildman CM, Attwell D (2010) The energy use associated with neural computation in the cerebellum. Journal of cerebral blood flow and metabolism : official journal of the International Society of Cerebral Blood Flow and Metabolism 30:403-414.

Hritcu L, Ciobica A, Stefan M, Mihasan M, Palamiuc L, Nabeshima T (2011) Spatial memory deficits and oxidative stress damage following exposure to lipopolysaccharide in a rodent model of Parkinson's disease. Neurosci Res 71:35-43.

Hu H, Jun S, Sarkar SN, Doll DN, Ren X, Simpkins JW (2014) Dynamic blood-brain barrier openings concur with increasing of miR-let7a in murine experimental stroke. Society for Neuroscience 701.05. 
Huang Y, Erdmann N, Peng H, Zhao Y, Zheng J (2005) The role of TNF related apoptosisinducing ligand in neurodegenerative diseases. Cellular \& molecular immunology 2:113122.

Huang ZR, Yu LP, Yang XC, Zhang F, Chen YR, Feng F, Qian XS, Cai J (2012) Human cytomegalovirus linked to stroke in a Chinese population. CNS Neurosci Ther 18:457460.

Hunt BJ, Jurd KM (1997) Relation between endothelial-cell activation and infection, inflammation, and infarction. Lancet 350:293.

Iadecola C, Anrather J (2011) The immunology of stroke: from mechanisms to translation. Nature medicine 17:796-808.

Ingraham JP, Forbes ME, Riddle DR, Sonntag WE (2008) Aging reduces hypoxia-induced microvascular growth in the rodent hippocampus. J Gerontol A Biol Sci Med Sci 63:1220.

Intiso D, Stampatore P, Zarrelli MM, Guerra GL, Arpaia G, Simone P, Tonali P, Beghi E (2003) Incidence of first-ever ischemic and hemorrhagic stroke in a well-defined community of southern Italy, 1993-1995. European journal of neurology : the official journal of the European Federation of Neurological Societies 10:559-565.

Jahani-Asl A, Cheung EC, Neuspiel M, MacLaurin JG, Fortin A, Park DS, McBride HM, Slack RS (2007) Mitofusin 2 protects cerebellar granule neurons against injury-induced cell death. The Journal of biological chemistry 282:23788-23798.

Jauch EC, Lindsell C, Broderick J, Fagan SC, Tilley BC, Levine SR (2006) Association of serial biochemical markers with acute ischemic stroke: the National Institute of Neurological Disorders and Stroke recombinant tissue plasminogen activator Stroke Study. Stroke; a journal of cerebral circulation 37:2508-2513.

Jensen HA, Loukogeorgakis S, Yannopoulos F, Rimpilainen E, Petzold A, Tuominen H, Lepola P, Macallister RJ, Deanfield JE, Makela T, Alestalo K, Kiviluoma K, Anttila V, Tsang V, Juvonen T (2011) Remote ischemic preconditioning protects the brain against injury after hypothermic circulatory arrest. Circulation 123:714-721.

Johnson GL, Nakamura K (2007) The c-jun kinase/stress-activated pathway: regulation, function and role in human disease. Biochimica et biophysica acta 1773:1341-1348.

Johnson JL, George SJ, Newby AC, Jackson CL (2005) Divergent effects of matrix metalloproteinases $3,7,9$, and 12 on atherosclerotic plaque stability in mouse brachiocephalic arteries. Proc Natl Acad Sci U S A 102:15575-15580.

Jung ME, Ju X, Simpkins JW, Metzger DB, Yan LJ, Wen Y (2011) Ethanol withdrawal acts as an age-specific stressor to activate cerebellar p38 kinase. Neurobiology of aging 32:22662278.

Keppel G, and Wickens TD. (2004) Design and Analysis A Researcher's Handbook. 4:194,201.

Kim Y, Zhou P, Qian L, Chuang JZ, Lee J, Li C, Iadecola C, Nathan C, Ding A (2007) MyD88-5 links mitochondria, microtubules, and JNK3 in neurons and regulates neuronal survival. The Journal of experimental medicine 204:2063-2074.

Kitagawa K, Matsumoto M, Mabuchi T, Yagita Y, Ohtsuki T, Hori M, Yanagihara T (1998) Deficiency of intercellular adhesion molecule 1 attenuates microcirculatory disturbance and infarction size in focal cerebral ischemia. Journal of cerebral blood flow and metabolism : official journal of the International Society of Cerebral Blood Flow and Metabolism 18:1336-1345. 
Konsman JP, Vigues S, Mackerlova L, Bristow A, Blomqvist A (2004) Rat brain vascular distribution of interleukin-1 type-1 receptor immunoreactivity: relationship to patterns of inducible cyclooxygenase expression by peripheral inflammatory stimuli. The Journal of comparative neurology 472:113-129.

Korherr C, Hofmeister R, Wesche H, Falk W (1997) A critical role for interleukin-1 receptor accessory protein in interleukin-1 signaling. European journal of immunology 27:262267.

Kotenko SV, Izotova LS, Pollack BP, Muthukumaran G, Paukku K, Silvennoinen O, Ihle JN, Pestka S (1996) Other kinases can substitute for Jak2 in signal transduction by interferongamma. The Journal of biological chemistry 271:17174-17182.

Krause CD, Mei E, Xie J, Jia Y, Bopp MA, Hochstrasser RM, Pestka S (2002) Seeing the light: preassembly and ligand-induced changes of the interferon gamma receptor complex in cells. Molecular \& cellular proteomics : MCP 1:805-815.

Kunz A, Park L, Abe T, Gallo EF, Anrather J, Zhou P, Iadecola C (2007) Neurovascular protection by ischemic tolerance: role of nitric oxide and reactive oxygen species. The Journal of neuroscience : the official journal of the Society for Neuroscience 27:70837093.

Lackland DT, Roccella EJ, Deutsch AF, Fornage M, George MG, Howard G, Kissela BM, Kittner SJ, Lichtman JH, Lisabeth LD, Schwamm LH, Smith EE, Towfighi A (2014) Factors influencing the decline in stroke mortality: a statement from the American Heart Association/American Stroke Association. Stroke 45:315-353.

Lambertsen KL, Gregersen R, Finsen B (2002) Microglial-macrophage synthesis of tumor necrosis factor after focal cerebral ischemia in mice is strain dependent. Journal of cerebral blood flow and metabolism : official journal of the International Society of Cerebral Blood Flow and Metabolism 22:785-797.

Lambertsen KL, Biber K, Finsen B (2012) Inflammatory cytokines in experimental and human stroke. Journal of cerebral blood flow and metabolism : official journal of the International Society of Cerebral Blood Flow and Metabolism 32:1677-1698.

Lambertsen KL, Clausen BH, Babcock AA, Gregersen R, Fenger C, Nielsen HH, Haugaard LS, Wirenfeldt M, Nielsen M, Dagnaes-Hansen F, Bluethmann H, Faergeman NJ, Meldgaard M, Deierborg T, Finsen B (2009) Microglia protect neurons against ischemia by synthesis of tumor necrosis factor. The Journal of neuroscience : the official journal of the Society for Neuroscience 29:1319-1330.

Lavine SD, Hofman FM, Zlokovic BV (1998) Circulating antibody against tumor necrosis factor-alpha protects rat brain from reperfusion injury. Journal of cerebral blood flow and metabolism : official journal of the International Society of Cerebral Blood Flow and Metabolism 18:52-58.

Lehnardt S, Massillon L, Follett P, Jensen FE, Ratan R, Rosenberg PA, Volpe JJ, Vartanian T (2003) Activation of innate immunity in the CNS triggers neurodegeneration through a Toll-like receptor 4-dependent pathway. Proc Natl Acad Sci U S A 100:8514-8519.

Li HL, Kostulas N, Huang YM, Xiao BG, van der Meide P, Kostulas V, Giedraitas V, Link H (2001) IL-17 and IFN-gamma mRNA expression is increased in the brain and systemically after permanent middle cerebral artery occlusion in the rat. Journal of neuroimmunology 116:5-14. 
Li L, Messas E, Batista EL, Jr., Levine RA, Amar S (2002) Porphyromonas gingivalis infection accelerates the progression of atherosclerosis in a heterozygous apolipoprotein Edeficient murine model. Circulation 105:861-867.

Liesz A, Suri-Payer E, Veltkamp C, Doerr H, Sommer C, Rivest S, Giese T, Veltkamp R (2009) Regulatory $\mathrm{T}$ cells are key cerebroprotective immunomodulators in acute experimental stroke. Nature medicine 15:192-199.

Lio D, Candore G, Crivello A, Scola L, Colonna-Romano G, Cavallone L, Hoffmann E, Caruso M, Licastro F, Caldarera CM, Branzi A, Franceschi C, Caruso C (2004) Opposite effects of interleukin 10 common gene polymorphisms in cardiovascular diseases and in successful ageing: genetic background of male centenarians is protective against coronary heart disease. J Med Genet 41:790-794.

Liu T, Clark RK, McDonnell PC, Young PR, White RF, Barone FC, Feuerstein GZ (1994) Tumor necrosis factor-alpha expression in ischemic neurons. Stroke; a journal of cerebral circulation 25:1481-1488.

Liu T, McDonnell PC, Young PR, White RF, Siren AL, Hallenbeck JM, Barone FC, Feurestein GZ (1993) Interleukin-1 beta mRNA expression in ischemic rat cortex. Stroke; a journal of cerebral circulation 24:1746-1750; discussion 1750-1741.

Liu ZG, Hsu H, Goeddel DV, Karin M (1996) Dissection of TNF receptor 1 effector functions: JNK activation is not linked to apoptosis while NF-kappaB activation prevents cell death. Cell 87:565-576.

Loddick SA, Rothwell NJ (1996) Neuroprotective effects of human recombinant interleukin-1 receptor antagonist in focal cerebral ischaemia in the rat. Journal of cerebral blood flow and metabolism : official journal of the International Society of Cerebral Blood Flow and Metabolism 16:932-940.

Loddick SA, Turnbull AV, Rothwell NJ (1998) Cerebral interleukin-6 is neuroprotective during permanent focal cerebral ischemia in the rat. Journal of cerebral blood flow and metabolism : official journal of the International Society of Cerebral Blood Flow and Metabolism 18:176-179.

Maddahi A, Kruse LS, Chen QW, Edvinsson L (2011) The role of tumor necrosis factor-alpha and TNF-alpha receptors in cerebral arteries following cerebral ischemia in rat. Journal of neuroinflammation 8:107.

Mann MK, Maresz K, Shriver LP, Tan Y, Dittel BN (2007) B cell regulation of CD4+CD25+ T regulatory cells and IL-10 via B7 is essential for recovery from experimental autoimmune encephalomyelitis. Journal of immunology (Baltimore, Md : 1950) 178:3447-3456.

Markgraf CG, Green EJ, Watson B, McCabe PM, Schneiderman N, Dietrich WD, Ginsberg MD (1994) Recovery of sensorimotor function after distal middle cerebral artery photothrombotic occlusion in rats. Stroke 25:153-159.

Marsh B, Stevens SL, Packard AE, Gopalan B, Hunter B, Leung PY, Harrington CA, StenzelPoore MP (2009) Systemic lipopolysaccharide protects the brain from ischemic injury by reprogramming the response of the brain to stroke: a critical role for IRF3. The Journal of neuroscience : the official journal of the Society for Neuroscience 29:9839-9849.

Marz P, Gadient RA, Otten U (1996) Expression of interleukin-6 receptor (IL-6R) and gp130 mRNA in PC12 cells and sympathetic neurons: modulation by tumor necrosis factor alpha (TNF-alpha). Brain research 706:71-79. 
Matsumoto H, Kumon Y, Watanabe H, Ohnishi T, Takahashi H, Imai Y, Tanaka J (2007) Expression of CD200 by macrophage-like cells in ischemic core of rat brain after transient middle cerebral artery occlusion. Neuroscience letters 418:44-48.

Mazzotta G, Sarchielli P, Caso V, Paciaroni M, Floridi A, Floridi A, Gallai V (2004) Different cytokine levels in thrombolysis patients as predictors for clinical outcome. European journal of neurology : the official journal of the European Federation of Neurological Societies 11:377-381.

McColl BW, Rothwell NJ, Allan SM (2007) Systemic inflammatory stimulus potentiates the acute phase and $\mathrm{CXC}$ chemokine responses to experimental stroke and exacerbates brain damage via interleukin-1- and neutrophil-dependent mechanisms. The Journal of neuroscience : the official journal of the Society for Neuroscience 27:4403-4412.

McColl BW, Allan SM, Rothwell NJ (2009) Systemic infection, inflammation and acute ischemic stroke. Neuroscience 158:1049-1061.

McCoy MK, Tansey MG (2008) TNF signaling inhibition in the CNS: implications for normal brain function and neurodegenerative disease. Journal of neuroinflammation 5:45.

McGill JK, Gallagher L, Carswell HV, Irving EA, Dominiczak AF, Macrae IM (2005) Impaired functional recovery after stroke in the stroke-prone spontaneously hypertensive rat. Stroke; a journal of cerebral circulation 36:135-141.

Mehta SL, Manhas N, Raghubir R (2007) Molecular targets in cerebral ischemia for developing novel therapeutics. Brain Res Rev 54:34-66.

Melani A, Turchi D, Vannucchi MG, Cipriani S, Gianfriddo M, Pedata F (2005) ATP extracellular concentrations are increased in the rat striatum during in vivo ischemia. Neurochem Int 47:442-448.

Mergenthaler P, Lindauer U, Dienel GA, Meisel A (2013) Sugar for the brain: the role of glucose in physiological and pathological brain function. Trends Neurosci 36:587-597.

Michelsen KS, Doherty TM, Shah PK, Arditi M (2004) TLR signaling: an emerging bridge from innate immunity to atherogenesis. J Immunol 173:5901-5907.

Mink JW, Blumenschine RJ, Adams DB (1981) Ratio of central nervous system to body metabolism in vertebrates: its constancy and functional basis. Am J Physiol 241:R203212.

Montaner J, Rovira A, Molina CA, Arenillas JF, Ribo M, Chacon P, Monasterio J, AlvarezSabin J (2003) Plasmatic level of neuroinflammatory markers predict the extent of diffusion-weighted image lesions in hyperacute stroke. Journal of cerebral blood flow and metabolism : official journal of the International Society of Cerebral Blood Flow and Metabolism 23:1403-1407.

Morris RG, Garrud P, Rawlins JN, O'Keefe J (1982) Place navigation impaired in rats with hippocampal lesions. Nature 297:681-683.

Moskowitz MA, Lo EH, Iadecola C (2010) The science of stroke: mechanisms in search of treatments. Neuron 67:181-198.

Murakami Y, Saito K, Hara A, Zhu Y, Sudo K, Niwa M, Fujii H, Wada H, Ishiguro H, Mori H, Seishima M (2005) Increases in tumor necrosis factor-alpha following transient global cerebral ischemia do not contribute to neuron death in mouse hippocampus. Journal of neurochemistry 93:1616-1622.

Nag S, Kapadia A, Stewart DJ (2011) Review: molecular pathogenesis of blood-brain barrier breakdown in acute brain injury. Neuropathology and applied neurobiology 37:3-23. 
Navarro A, Sanchez Del Pino MJ, Gomez C, Peralta JL, Boveris A (2002) Behavioral dysfunction, brain oxidative stress, and impaired mitochondrial electron transfer in aging mice. Am J Physiol Regul Integr Comp Physiol 282:R985-992.

Nawashiro H, Martin D, Hallenbeck JM (1997a) Inhibition of tumor necrosis factor and amelioration of brain infarction in mice. Journal of cerebral blood flow and metabolism : official journal of the International Society of Cerebral Blood Flow and Metabolism 17:229-232.

Nawashiro H, Martin D, Hallenbeck JM (1997b) Neuroprotective effects of TNF binding protein in focal cerebral ischemia. Brain research 778:265-271.

Nawashiro H, Tasaki K, Ruetzler CA, Hallenbeck JM (1997c) TNF-alpha pretreatment induces protective effects against focal cerebral ischemia in mice. Journal of cerebral blood flow and metabolism : official journal of the International Society of Cerebral Blood Flow and Metabolism 17:483-490.

Nayak AR, Kashyap RS, Kabra D, Purohit HJ, Taori GM, Daginawala HF (2012) Time course of inflammatory cytokines in acute ischemic stroke patients and their relation to inter-alfa trypsin inhibitor heavy chain 4 and outcome. Annals of Indian Academy of Neurology 15:181-185.

Ohtaki H, Takaki A, Yin L, Dohi K, Nakamachi T, Matsunaga M, Horai R, Asano M, Iwakura Y, Shioda S (2003) Suppression of oxidative stress after transient focal ischemia in interleukin-1 knock out mice. Acta neurochirurgica Supplement 86:191-194.

Ohtsuki T, Ruetzler CA, Tasaki K, Hallenbeck JM (1996) Interleukin-1 mediates induction of tolerance to global ischemia in gerbil hippocampal CA1 neurons. Journal of cerebral blood flow and metabolism : official journal of the International Society of Cerebral Blood Flow and Metabolism 16:1137-1142.

Olton DS, Becker, J. T., Handelmann, G. E. (1979) Hippocampus, space, and memory. Behav Brain Res 2:313-322.

Ooboshi H, Ibayashi S, Shichita T, Kumai Y, Takada J, Ago T, Arakawa S, Sugimori H, Kamouchi M, Kitazono T, Iida M (2005) Postischemic gene transfer of interleukin-10 protects against both focal and global brain ischemia. Circulation 111:913-919.

Ormstad H, Aass HC, Amthor KF, Lund-Sorensen N, Sandvik L (2011a) Serum cytokine and glucose levels as predictors of poststroke fatigue in acute ischemic stroke patients. Journal of neurology 258:670-676.

Ormstad H, Aass HC, Lund-Sorensen N, Amthor KF, Sandvik L (2011b) Serum levels of cytokines and C-reactive protein in acute ischemic stroke patients, and their relationship to stroke lateralization, type, and infarct volume. Journal of neurology 258:677-685.

Painsipp E, Kofer MJ, Sinner F, Holzer P (2011) Prolonged depression-like behavior caused by immune challenge: influence of mouse strain and social environment. PLoS One 6:e20719.

Palasik W, Fiszer U, Lechowicz W, Czartoryska B, Krzesiewicz M, Lugowska A (2005) Assessment of relations between clinical outcome of ischemic stroke and activity of inflammatory processes in the acute phase based on examination of selected parameters. European neurology 53:188-193.

Perez-de Puig I, Miro F, Salas-Perdomo A, Bonfill-Teixidor E, Ferrer-Ferrer M, MarquezKisinousky L, Planas AM (2013) IL-10 deficiency exacerbates the brain inflammatory response to permanent ischemia without preventing resolution of the lesion. Journal of 
cerebral blood flow and metabolism : official journal of the International Society of Cerebral Blood Flow and Metabolism 33:1955-1966.

Perini F, Morra M, Alecci M, Galloni E, Marchi M, Toso V (2001) Temporal profile of serum anti-inflammatory and pro-inflammatory interleukins in acute ischemic stroke patients. Neurological sciences : official journal of the Italian Neurological Society and of the Italian Society of Clinical Neurophysiology 22:289-296.

Pradillo JM, Romera C, Hurtado O, Cardenas A, Moro MA, Leza JC, Davalos A, Castillo J, Lorenzo P, Lizasoain I (2005) TNFR1 upregulation mediates tolerance after brain ischemic preconditioning. Journal of cerebral blood flow and metabolism : official journal of the International Society of Cerebral Blood Flow and Metabolism 25:193-203.

Reimann-Philipp U, Ovase R, Weigel PH, Grammas P (2001) Mechanisms of cell death in primary cortical neurons and PC12 cells. Journal of neuroscience research 64:654-660.

Relton JK, Martin D, Thompson RC, Russell DA (1996) Peripheral administration of Interleukin-1 Receptor antagonist inhibits brain damage after focal cerebral ischemia in the rat. Experimental neurology 138:206-213.

Ren X, Akiyoshi K, Dziennis S, Vandenbark AA, Herson PS, Hurn PD, Offner H (2011) Regulatory B cells limit CNS inflammation and neurologic deficits in murine experimental stroke. The Journal of neuroscience : the official journal of the Society for Neuroscience 31:8556-8563.

Rogers DC, Campbell CA, Stretton JL, Mackay KB (1997) Correlation between motor impairment and infarct volume after permanent and transient middle cerebral artery occlusion in the rat. Stroke 28:2060-2065; discussion 2066.

Rubin LL, Staddon JM (1999) The cell biology of the blood-brain barrier. Annual review of neuroscience 22:11-28.

Rudd JH, Warburton EA, Fryer TD, Jones HA, Clark JC, Antoun N, Johnstrom P, Davenport AP, Kirkpatrick PJ, Arch BN, Pickard JD, Weissberg PL (2002) Imaging atherosclerotic plaque inflammation with $[18 \mathrm{~F}]$-fluorodeoxyglucose positron emission tomography. Circulation 105:2708-2711.

Sairanen T, Carpen O, Karjalainen-Lindsberg ML, Paetau A, Turpeinen U, Kaste M, Lindsberg PJ (2001) Evolution of cerebral tumor necrosis factor-alpha production during human ischemic stroke. Stroke; a journal of cerebral circulation 32:1750-1758.

Saito M, Yoshida K, Hibi M, Taga T, Kishimoto T (1992) Molecular cloning of a murine IL-6 receptor-associated signal transducer, gp130, and its regulated expression in vivo. Journal of immunology (Baltimore, Md : 1950) 148:4066-4071.

Sandoval KE, Witt KA (2008) Blood-brain barrier tight junction permeability and ischemic stroke. Neurobiology of disease 32:200-219.

Sas K, Robotka H, Toldi J, Vecsei L (2007) Mitochondria, metabolic disturbances, oxidative stress and the kynurenine system, with focus on neurodegenerative disorders. Journal of the neurological sciences 257:221-239.

Schaar KL, Brenneman MM, Savitz SI (2010) Functional assessments in the rodent stroke model. Experimental \& translational stroke medicine 2:13.

Schock SC, Munyao N, Yakubchyk Y, Sabourin LA, Hakim AM, Ventureyra EC, Thompson CS (2007) Cortical spreading depression releases ATP into the extracellular space and purinergic receptor activation contributes to the induction of ischemic tolerance. Brain research 1168:129-138. 
Selakovic V, Colic M, Jovanovic M, Raicevic R, Jovicic A (2003) Cerebrospinal fluid and plasma concentration of soluble intercellular adhesion molecule 1, vascular cell adhesion molecule 1 and endothelial leukocyte adhesion molecule in patients with acute ischemic brain disease. Vojnosanit Pregl 60:139-146.

Simpkins JW, Yi KD, Yang SH, Dykens JA (2010) Mitochondrial mechanisms of estrogen neuroprotection. Biochimica et biophysica acta 1800:1113-1120.

Sims NR, Anderson MF (2002) Mitochondrial contributions to tissue damage in stroke. Neurochemistry international 40:511-526.

Sims NR, Muyderman H (2010) Mitochondria, oxidative metabolism and cell death in stroke. Biochimica et biophysica acta 1802:80-91.

Smeeth L, Thomas SL, Hall AJ, Hubbard R, Farrington P, Vallance P (2004) Risk of myocardial infarction and stroke after acute infection or vaccination. N Engl J Med 351:2611-2618.

Smith CJ, Lawrence CB, Rodriguez-Grande B, Kovacs KJ, Pradillo JM, Denes A (2013) The immune system in stroke: clinical challenges and their translation to experimental research. Journal of neuroimmune pharmacology : the official journal of the Society on NeuroImmune Pharmacology 8:867-887.

Sotgiu S, Zanda B, Marchetti B, Fois ML, Arru G, Pes GM, Salaris FS, Arru A, Pirisi A, Rosati $\mathrm{G}$ (2006) Inflammatory biomarkers in blood of patients with acute brain ischemia. European journal of neurology : the official journal of the European Federation of Neurological Societies 13:505-513.

Spera PA, Ellison JA, Feuerstein GZ, Barone FC (1998) IL-10 reduces rat brain injury following focal stroke. Neuroscience letters 251:189-192.

Stavnezer AJ, Hyde LA, Bimonte HA, Armstrong CM, Denenberg VH (2002) Differential learning strategies in spatial and nonspatial versions of the Morris water maze in the C57BL/6J inbred mouse strain. Behav Brain Res 133:261-270.

Steckley D, Karajgikar M, Dale LB, Fuerth B, Swan P, Drummond-Main C, Poulter MO, Ferguson SS, Strasser A, Cregan SP (2007) Puma is a dominant regulator of oxidative stress induced Bax activation and neuronal apoptosis. The Journal of neuroscience : the official journal of the Society for Neuroscience 27:12989-12999.

Subramanian S, Zhang B, Kosaka Y, Burrows GG, Grafe MR, Vandenbark AA, Hurn PD, Offner H (2009) Recombinant T cell receptor ligand treats experimental stroke. Stroke; a journal of cerebral circulation 40:2539-2545.

Suzuki S, Tanaka K, Nogawa S, Nagata E, Ito D, Dembo T, Fukuuchi Y (1999) Temporal profile and cellular localization of interleukin-6 protein after focal cerebral ischemia in rats. Journal of cerebral blood flow and metabolism : official journal of the International Society of Cerebral Blood Flow and Metabolism 19:1256-1262.

Taheri S, Candelario-Jalil E, Estrada EY, Rosenberg GA (2009) Spatiotemporal correlations between blood-brain barrier permeability and apparent diffusion coefficient in a rat model of ischemic stroke. PloS one 4:e6597.

Tajes M, Ramos-Fernandez E, Weng-Jiang X, Bosch-Morato M, Guivernau B, Eraso-Pichot A, Salvador B, Fernandez-Busquets X, Roquer J, Munoz FJ (2014) The blood-brain barrier: structure, function and therapeutic approaches to cross it. Molecular membrane biology 31:152-167.

Tamatani M, Che YH, Matsuzaki H, Ogawa S, Okado H, Miyake S, Mizuno T, Tohyama M (1999) Tumor necrosis factor induces Bcl-2 and Bcl-x expression through NFkappaB 
activation in primary hippocampal neurons. The Journal of biological chemistry 274:8531-8538.

Tanaka M, Nakae S, Terry RD, Mokhtari GK, Gunawan F, Balsam LB, Kaneda H, Kofidis T, Tsao PS, Robbins RC (2004) Cardiomyocyte-specific Bcl-2 overexpression attenuates ischemia-reperfusion injury, immune response during acute rejection, and graft coronary artery disease. Blood 104:3789-3796.

Tancredi V, D'Antuono M, Cafe C, Giovedi S, Bue MC, D'Arcangelo G, Onofri F, Benfenati F (2000) The inhibitory effects of interleukin-6 on synaptic plasticity in the rat hippocampus are associated with an inhibition of mitogen-activated protein kinase ERK. Journal of neurochemistry 75:634-643.

Tang SC, Arumugam TV, Xu X, Cheng A, Mughal MR, Jo DG, Lathia JD, Siler DA, Chigurupati S, Ouyang X, Magnus T, Camandola S, Mattson MP (2007) Pivotal role for neuronal Toll-like receptors in ischemic brain injury and functional deficits. Proceedings of the National Academy of Sciences of the United States of America 104:13798-13803.

Tanner CM, Kamel F, Ross GW, Hoppin JA, Goldman SM, Korell M, Marras C, Bhudhikanok GS, Kasten M, Chade AR, Comyns K, Richards MB, Meng C, Priestley B, Fernandez HH, Cambi F, Umbach DM, Blair A, Sandler DP, Langston JW (2011) Rotenone, paraquat, and Parkinson's disease. Environmental health perspectives 119:866-872.

Tarkowski E, Rosengren L, Blomstrand C, Wikkelso C, Jensen C, Ekholm S, Tarkowski A (1995) Early intrathecal production of interleukin-6 predicts the size of brain lesion in stroke. Stroke; a journal of cerebral circulation 26:1393-1398.

Tarkowski E, Rosengren L, Blomstrand C, Wikkelso C, Jensen C, Ekholm S, Tarkowski A (1997) Intrathecal release of pro- and anti-inflammatory cytokines during stroke. Clinical and experimental immunology 110:492-499.

Tarr AJ, McLinden KA, Kranjac D, Kohman RA, Amaral W, Boehm GW (2011) The effects of age on lipopolysaccharide-induced cognitive deficits and interleukin-1beta expression. Behav Brain Res 217:481-485.

Taylor RC, Cullen SP, Martin SJ (2008) Apoptosis: controlled demolition at the cellular level. Nat Rev Mol Cell Biol 9:231-241.

Terao S, Yilmaz G, Stokes KY, Ishikawa M, Kawase T, Granger DN (2008) Inflammatory and injury responses to ischemic stroke in obese mice. Stroke; a journal of cerebral circulation 39:943-950.

Thornberry NA, Bull HG, Calaycay JR, Chapman KT, Howard AD, Kostura MJ, Miller DK, Molineaux SM, Weidner JR, Aunins J, et al. (1992) A novel heterodimeric cysteine protease is required for interleukin-1 beta processing in monocytes. Nature 356:768-774.

van der Poll T, Buller HR, ten Cate H, Wortel CH, Bauer KA, van Deventer SJ, Hack CE, Sauerwein HP, Rosenberg RD, ten Cate JW (1990) Activation of coagulation after administration of tumor necrosis factor to normal subjects. N Engl J Med 322:1622-1627.

van der Poll T, Levi M, Hack CE, ten Cate H, van Deventer SJ, Eerenberg AJ, de Groot ER, Jansen J, Gallati H, Buller HR, et al. (1994) Elimination of interleukin 6 attenuates coagulation activation in experimental endotoxemia in chimpanzees. $\mathrm{J}$ Exp Med 179:1253-1259.

Vila N, Castillo J, Davalos A, Chamorro A (2000a) Proinflammatory cytokines and early neurological worsening in ischemic stroke. Stroke; a journal of cerebral circulation $31: 2325-2329$. 
Vila N, Reverter JC, Yague J, Chamorro A (2000b) Interaction between interleukin-6 and the natural anticoagulant system in acute stroke. J Interferon Cytokine Res 20:325-329.

Vila N, Castillo J, Davalos A, Esteve A, Planas AM, Chamorro A (2003) Levels of antiinflammatory cytokines and neurological worsening in acute ischemic stroke. Stroke; a journal of cerebral circulation 34:671-675.

Viviani B, Bartesaghi S, Gardoni F, Vezzani A, Behrens MM, Bartfai T, Binaglia M, Corsini E, Di Luca M, Galli CL, Marinovich M (2003) Interleukin-1beta enhances NMDA receptormediated intracellular calcium increase through activation of the Src family of kinases. The Journal of neuroscience : the official journal of the Society for Neuroscience 23:8692-8700.

Vosler PS, Graham SH, Wechsler LR, Chen J (2009) Mitochondrial targets for stroke: focusing basic science research toward development of clinically translatable therapeutics. Stroke; a journal of cerebral circulation 40:3149-3155.

Wajant H, Pfizenmaier K, Scheurich P (2003) Tumor necrosis factor signaling. Cell death and differentiation 10:45-65.

Waje-Andreassen U, Krakenes J, Ulvestad E, Thomassen L, Myhr KM, Aarseth J, Vedeler CA (2005) IL-6: an early marker for outcome in acute ischemic stroke. Acta neurologica Scandinavica 111:360-365.

Wallach D, Arumugam TU, Boldin MP, Cantarella G, Ganesh KA, Goltsev Y, Goncharov TM, Kovalenko AV, Rajput A, Varfolomeev EE, Zhang SQ (2002) How are the regulators regulated? The search for mechanisms that impose specificity on induction of cell death and NF-kappaB activation by members of the TNF/NGF receptor family. Arthritis research 4 Suppl 3:S189-196.

Wang Q, Tang XN, Yenari MA (2007a) The inflammatory response in stroke. J Neuroimmunol 184:53-68.

Wang X, Yue TL, Young PR, Barone FC, Feuerstein GZ (1995) Expression of interleukin-6, cfos, and zif268 mRNAs in rat ischemic cortex. Journal of cerebral blood flow and metabolism : official journal of the International Society of Cerebral Blood Flow and Metabolism 15:166-171.

Wang X, Yue TL, Barone FC, White RF, Gagnon RC, Feuerstein GZ (1994) Concomitant cortical expression of TNF-alpha and IL-1 beta mRNAs follows early response gene expression in transient focal ischemia. Molecular and chemical neuropathology / sponsored by the International Society for Neurochemistry and the World Federation of Neurology and research groups on neurochemistry and cerebrospinal fluid 23:103-114.

Wang XT, Pei DS, Xu J, Guan QH, Sun YF, Liu XM, Zhang GY (2007b) Opposing effects of Bad phosphorylation at two distinct sites by Akt1 and JNK1/2 on ischemic brain injury. Cell Signal 19:1844-1856.

Wang ZW, Li Y, Huang LY, Guan QK, Xu da W, Zhou WK, Zhang XZ (2012) Helicobacter pylori infection contributes to high risk of ischemic stroke: evidence from a metaanalysis. J Neurol 259:2527-2537.

Warach S, Latour LL (2004) Evidence of reperfusion injury, exacerbated by thrombolytic therapy, in human focal brain ischemia using a novel imaging marker of early bloodbrain barrier disruption. Stroke; a journal of cerebral circulation 35:2659-2661.

Warburton L, Gillard J (2006) Functional imaging of carotid atheromatous plaques. J Neuroimaging 16:293-301. 
Westendorp WF, Nederkoorn PJ, Vermeij JD, Dijkgraaf MG, van de Beek D (2011) Post-stroke infection: a systematic review and meta-analysis. BMC neurology 11:110.

Winter B, Juckel G, Viktorov I, Katchanov J, Gietz A, Sohr R, Balkaya M, Hortnagl H, Endres M (2005) Anxious and hyperactive phenotype following brief ischemic episodes in mice. Biol Psychiatry 57:1166-1175.

Wong CH, Jenne CN, Lee WY, Leger C, Kubes P (2011) Functional innervation of hepatic iNKT cells is immunosuppressive following stroke. Science (New York, NY) 334:101105.

Works MG, Koenig JB, Sapolsky RM (2013) Soluble TNF receptor 1-secreting ex vivo-derived dendritic cells reduce injury after stroke. Journal of cerebral blood flow and metabolism : official journal of the International Society of Cerebral Blood Flow and Metabolism 33:1376-1385.

Yamasaki Y, Matsuura N, Shozuhara H, Onodera H, Itoyama Y, Kogure K (1995) Interleukin-1 as a pathogenetic mediator of ischemic brain damage in rats. Stroke; a journal of cerebral circulation 26:676-680; discussion 681.

Yang GY, Gong C, Qin Z, Liu XH, Lorris Betz A (1999) Tumor necrosis factor alpha expression produces increased blood-brain barrier permeability following temporary focal cerebral ischemia in mice. Brain research Molecular brain research 69:135-143.

Yang Y, Rosenberg GA (2011) Blood-brain barrier breakdown in acute and chronic cerebrovascular disease. Stroke; a journal of cerebral circulation 42:3323-3328.

Yenari MA, Kunis D, Sun GH, Onley D, Watson L, Turner S, Whitaker S, Steinberg GK (1998) $\mathrm{Hu} 23 \mathrm{~F} 2 \mathrm{G}$, an antibody recognizing the leukocyte CD11/CD18 integrin, reduces injury in a rabbit model of transient focal cerebral ischemia. Exp Neurol 153:223-233.

Yin W, Cao G, Johnnides MJ, Signore AP, Luo Y, Hickey RW, Chen J (2006) TAT-mediated delivery of Bcl-xL protein is neuroprotective against neonatal hypoxic-ischemic brain injury via inhibition of caspases and AIF. Neurobiol Dis 21:358-371.

Youle RJ, Strasser A (2008) The BCL-2 protein family: opposing activities that mediate cell death. Nat Rev Mol Cell Biol 9:47-59.

Zaremba J, Losy J (2001) Early TNF-alpha levels correlate with ischaemic stroke severity. Acta neurologica Scandinavica 104:288-295.

Zhang Q, Raoof M, Chen Y, Sumi Y, Sursal T, Junger W, Brohi K, Itagaki K, Hauser CJ (2010) Circulating mitochondrial DAMPs cause inflammatory responses to injury. Nature 464:104-107.

Zhao X, Bausano B, Pike BR, Newcomb-Fernandez JK, Wang KK, Shohami E, Ringger NC, DeFord SM, Anderson DK, Hayes RL (2001) TNF-alpha stimulates caspase-3 activation and apoptotic cell death in primary septo-hippocampal cultures. Journal of neuroscience research 64:121-131.

Zheng Z, Yenari MA (2004) Post-ischemic inflammation: molecular mechanisms and therapeutic implications. Neurological research 26:884-892.

Zhou X, Nicoletti A, Elhage R, Hansson GK (2000) Transfer of CD4(+) T cells aggravates atherosclerosis in immunodeficient apolipoprotein $\mathrm{E}$ knockout mice. Circulation 102:2919-2922.

Zhu XH, Qiao H, Du F, Xiong Q, Liu X, Zhang X, Ugurbil K, Chen W (2012) Quantitative imaging of energy expenditure in human brain. NeuroImage 60:2107-2117. 
Zurru MC, Alonzo C, Brescacin L, Romano M, Camera LA, Waisman G, Cristiano E, Ovbiagele B (2009) Recent respiratory infection predicts atherothrombotic stroke: case-control study in a Buenos Aires healthcare system. Stroke 40:1986-1990. 